\title{
FERMIONIC STRUCTURE IN THE SINE-GORDON MODEL: FORM FACTORS AND NULL-VECTORS
}

\author{
M. JIMBO, T. MIWA AND F. SMIRNOV
}

\begin{abstract}
The form factor bootstrap in integrable quantum field theory allows one to capture local fields in terms of infinite sequences of Laurent polynomials called 'towers'. For the sine-Gordon model, towers are systematically described by fermions introduced some time ago by Babelon, Bernard and Smirnov. Recently the authors developed a new method for evaluating one-point functions of descendant fields, using yet another fermions which act on the space of local fields. The goal of this paper is to establish that these two fermions are one and the same object. This opens up a way for answering the longstanding question about how to identify precisely towers and local fields.
\end{abstract}

\section{INTRODUCTION}

The famous sine-Gordon ( $\mathrm{sG}$ ) model is described by the action

$$
\mathcal{A}^{\mathrm{sG}}=\int\left[\frac{1}{16 \pi}\left(\partial_{\mu} \boldsymbol{\varphi}(x)\right)^{2}+\frac{\boldsymbol{\mu}^{2}}{\sin \pi \beta^{2}} 2 \cos (\beta \boldsymbol{\varphi}(x))\right] d^{2} x .
$$

In this paper we use the parameter

$$
\nu=1-\beta^{2},
$$

following the convention in our previous works [1, 2, 3, 1,

In our opinion, the sG model is an ideal playground for developing new methods of Integrable Quantum Field Theory (IQFT). On the one hand, this model is sufficiently complicated. Its spectrum contains, together with usual particles and their bound states (breathers), topologically non-trivial particles: solitons. On the other hand, the model is intimately related with the simplest non-trivial quantum affine algebra $U_{q}\left(\widehat{\mathfrak{s l}}_{2}\right)$, so, its study does not involve purely technical complications coming from considering quantum groups of higher rank. The latter property is closely related to the fact that in the classical case the (quasi)-periodic solutions of the sG equation are related to hyper-elliptic Riemann surfaces, which represent the simplest, but still non-trivial example of algebraic curves. All that should be clearly understood by a researcher who is interested in serious investigation of the sG model.

During the period of rapid development of IQFT, several important results were obtained for the sG model. They include the discovery of the exact S-matrix [4, formulation of axioms for the form factors and solving equations constituting these axioms [5, 6, 7]. Around the same period there appeared the work [8] which is

\footnotetext{
${ }^{1}$ The parameter $\nu$ is related to $\xi=\xi^{\mathrm{FS}}$ in [7] and $\xi=\xi^{\mathrm{SL}}$ in $[9]$ by $\frac{1-\nu}{\nu}=\xi^{\mathrm{FS}} / \pi=\xi^{\mathrm{SL}}$.
} 
very important for us. This paper investigated the equivalence of the form factor bootstrap to the Operator Product Expansion (OPE) appearing in the context of Perturbed Conformal Field Theory (PCFT) combined with the knowledge of the one-point functions.

Let us discuss the form factors in the $\mathrm{sG}$ model. In the original works [5, 7] they were constructed for operators most relevant to physics: energy-momentum tensor, topological current and disorder operators. With the rapid development of Conformal Field Theory (CFT) which started with the famous paper [10], the following interesting question arose: Find the form factors of all the local operators which are described in the ultraviolet limit by CFT. The papers [11, 12] were important in understanding this problem together with the paper [8] cited before. Due to the latter paper it became clear that the ideal object for this study is not the space of descendants of the degenerate fields, but rather the space of descendants of the generic primary field

$$
\Phi_{\alpha}(z, \bar{z})=e^{i \alpha \frac{\nu}{2 \sqrt{1-\nu}} \varphi(z, \bar{z})},
$$

with arbitrary $\alpha$. The normalisation coefficient $\frac{\nu}{2 \sqrt{1-\nu}}$ is introduced for convenience. The point is that considering generic $\nu$ and $\alpha$ we avoid resonances, and the correspondence between the sG operators and their ultra-violet CFT counterparts becomes one-to-one. According to this logic, the first task is to compute the form factors of the exponential operators $\Phi_{\alpha}(0)$. This was done in the paper [9]. This paper required the knowledge of zero-particle form factors (one-point functions) in infinite volume found in [13]. It should be said also that the method of [9] originates in the algebraic study of correlation functions and form factors for lattice models [14].

It has been said that the $\mathrm{sG}$ model possesses the advantage of being sufficiently complicated while avoiding unnecessary difficulties of purely algebraic nature. Here we want to explain what the words "sufficiently complicated" mean. There are models of IQFT with much simpler, diagonal, S-matrices (sinh-Gordon model, Lee-Yang model, $Z_{N}$ model, and many others). For these models the form factor bootstrap considerably simplifies. However, to our mind it simplifies too much making obscure the mathematical structure of the solution. When the answer is simple it may allow different accidental representations. The $\mathrm{sG}$ model is sufficiently rigid for form factors. Irrespectively of the method used to derive them, one obtains essentially the same formulae given by a certain integral transformation. The integrals involved in this transformation can be understood as quantum deformation of the hyper-elliptic Abelian integrals [15]. The analogy with the classical case was crucial for realising this fact. While quite useful, this analogy is not so straightforward. In classical mathematics, when the solution to a differential equation is given by an integral transformation, different solutions are parametrised by different contours. After quantisation the differential equations are replaced by a certain Riemann-Hilbert problem (see Section 21), and the contours are replaced by polynomials which one can insert under the integral. These polynomials label different local operators. In this paper we consider only soliton form factors for the local operators which do not change the topological charge. So, the number of particles is even, say $2 n$. More precisely, with each $n$ there is associated a polynomial entering the integral formula 
for $2 n$-particle form factors, and these polynomials are mutually related by a certain recurrence relation. Hence a local operator is represented by an infinite sequence of such polynomials which we call a tower. The precise definition is given in Section 4 .

We would like to make one more historical comment. When the method of counting local fields became clear, the following important problem arose. It is well known that for special values of $\alpha$, which correspond to degenerate primary fields, some descendants vanish. Conventionally these vanishing descendants are called null vectors. On the other hand the number of towers corresponding to descendants of a primary field is independent of $\alpha$. The only solution to this apparent contradiction may consist in vanishing of the integrals defining the form factors in some cases. This is indeed the case. There are different reasons for integrals to vanish, the most important being the Riemann bilinear identity for quantum Abelian integrals [16].

Returning to the general case, let us fix a generic $\alpha$ and explain how the space of towers is organised. A convenient language for that was introduced in the paper [17]. We slightly generalise the results of this paper and change the notation. The local integrals of motion act on the local operators by commutators. The form factors obtained by this action are easy to compute, so, we shall ignore these descendants by local integrals of motion. The real problem is to describe the quotient space. To this end, following [17] we introduce the fermionic creation operators $\psi_{2 j-1}^{*}, \chi_{2 j-1}^{*}$, $\bar{\psi}_{2 j-1}^{*}, \bar{\chi}_{2 j-1}^{*}$ acting on towers, $j=1,2, \cdots$. The operators $\psi_{2 j-1}^{*}, \chi_{2 j-1}^{*}$ correspond to the right chirality, and the operators $\bar{\psi}_{2 j-1}^{*}, \bar{\chi}_{2 j-1}^{*}$ to the left chirality. We call these fermions Babelon-Bernard-Smirnov (BBS) fermions.

Let $M_{0}^{(\star)}=\left\{M^{(n)}\right\}_{n=0}^{\infty}$ denote the tower corresponding to the primary field $\Phi_{\alpha}$. The space of towers $L_{\mathcal{O}_{\alpha}}^{(\star)}$ corresponding to local operators $\mathcal{O}_{\alpha}$ is obtained acting on $M_{0}^{(\star)}$ by integrals of motion and BBS fermions. The latter must satisfy the restriction

$$
\#\left(\psi^{*}\right)+\#\left(\bar{\psi}^{*}\right)=\#\left(\chi^{*}\right)+\#\left(\bar{\chi}^{*}\right) .
$$

Later we shall say that the towers satisfying (1.3) have charge 0. It is also useful to introduce the weight of a local operator $\mathcal{O}_{\alpha}$ by

$$
m=\frac{1}{2}\left(\#\left(\psi^{*}\right)-\#\left(\chi^{*}\right)+\#\left(\bar{\chi}^{*}\right)-\#\left(\bar{\psi}^{*}\right)\right) .
$$

The local operators of weight $m$ correspond to $\Phi_{\alpha+2 m \frac{1-\nu}{\nu}}$, and its Virasoro descendants.

As we mentioned already, there is a one-to-one correspondence between the operators in the $\mathrm{sG}$ model and in the corresponding ultraviolet CFT. There is certain arbitrariness in choosing the latter. We prefer to split $2 \cos (\beta \varphi(x))$ in (1.1) into the sum $e^{-i \beta \varphi(x)}+e^{i \beta \varphi(x)}$ giving the first term to the CFT action and considering the second one as the perturbation. The CFT in question is nothing but the complex Liouville model. The fields $\Phi_{m \frac{1-\nu}{\nu}}$ are degenerate. For $m \geq 0$ they correspond to the fields from the first row of the Kac table. So, certain descendants of these fields must vanish. This circumstance was the main subject of [17]. Namely, it was shown that the Riemann bilinear identity and some additional simpler properties of quantum Abelian integrals imply certain relations between the fermionic descendants for the degenerate fields. This will be discussed in Section 11. Taking these null vectors into 
account, we arrive at the correct number of local operators. However an important question was left unanswered in [17]: to identify precisely the descendants by BBS fermions and the usual Virasoro descendants. We shall address this issue in this paper.

Being unable to solve this problem, the authors of [17] concentrated on the classical limit showing that the description of the null vectors in terms of the fermions provides a new formulation of the classical hierarchy. Actually, they considered only right chiral descendants, so, the hierarchy in question was that of the Korteweg-deVries $(\mathrm{KdV})$ equation. Another way of counting local operators was explained from a representation theory viewpoint in [18.

Now we would like to discuss a seemingly completely different subject. Several years ago, the present authors together with H. Boos and Y. Takeyama started a joint work on correlation functions for the XXZ spin chain. We have been motivated by a strong feeling that the formulae known at the time were quite unsatisfactory. As a result, we found a fermionic description of the space of quasi-local operators.

We believe our fermionic construction to be important, so, we would like to explain its intuitive meaning. Generally speaking, our understanding of quantum field theory is limited because very few exact results are known about models with interaction. Our intuition relies too heavily on free fields, and this can be sometimes misleading. To give an example in the context of the XXZ spin chain, the free model is the XX spin chain arising at a particular value of the coupling constant where the model becomes equivalent to the lattice Dirac fermion. In this case, one diagonalises the Hamiltonian introducing the creation-annihilation operators by Fourier transform. We parametrise the corresponding momentum as $i p=\log \frac{1-\zeta^{2}}{1+\zeta^{2}}$. On the other hand, the same $\zeta$-dependent creation operators can be used for constructing quasi-local operators from a given one: we just take the (anti)-commutators and develop the result around the point $\zeta^{2}=1$. So, the same construction with Fourier transform serves two different goals: diagonalising the Hamiltonian, and describing the space of quasi-local operators. It is important to understand that these two procedures are completely different for models with interaction. Moreover, before finding the fermionic description of the space of quasi-local fields we even did not know that the second procedure makes sense. Let us be more explicit about this point.

The diagonilisation of the Hamiltonian for integrable models in general, and the XXZ spin chain in particular, is achieved by the Bethe Ansatz which is best understood in its algebraic formulation (ABA) [19]. The ABA can be viewed as a highly non-linear analogue of Fourier transform in the space of states. It does not explicitly introduce the creation-annihilation operators, and cannot be used for creating the local fields by adjoint action as it was possible for the free-fermion case. The real achievement of our works [20, 21] is that we were able to find a set of creation operators $\mathbf{b}^{*}(\zeta), \mathbf{c}^{*}(\zeta)$ which act on the space of quasi-local operators creating this entire space from the "primary fields" by development around $\zeta^{2}=1$. It may be said that, comparing to ABA, we have introduced another non-linear Fourier transform in the space of operators. In doing that we used the same algebraic structures as the one 
used in ABA (quantum groups [22, 23] essentially) but in a more sophisticated way, including in particular the methods developed by [24].

Our fermionic operators have two notable features. First, they indeed act on the space of quasi-local operators, i.e., they respect locality. This was proved in the paper [21]. Second, the partition function of the the equivalent 6-vertex model, formulated on a cylinder with an insertion of a quasi-local operator created by the fermions, is expressed in terms of a single function $\omega(\zeta, \xi)$. This was proved in the paper [25]. We shall refer to the compact direction on the cylinder as the Matsubara direction.

Our next goal was to take the scaling limit in order to arrive at the $c<1$ CFT. After the transformation to fermions has been done this scaling limit is simple. In a certain sense we consider the fermionic construction for the lattice model as an existence theorem: the local operators are parameterised by parameters $\zeta$, and the partition function with insertion is expressed in terms of $\omega(\zeta, \xi)$. To consider the scaling limit it suffices to describe it for $\omega(\zeta, \xi)$. Here the TBA-like equations for $\omega(\zeta, \xi)$ [26] are very useful. (We use the term TBA in a broad sense. Actually the techniques used in [26, 1] is that of [27].) Comparing the scaling limit of $\omega(\zeta, \xi)$ with the CFT three-point functions, we find the relation between the description of local operators by the fermions and the one in terms of the Virasoro algebra.

For the application to the sG model, we use an inhomogeneous XXZ spin chain, and obtain in the scaling limit fermions $\boldsymbol{\beta}_{2 j-1}^{*}, \boldsymbol{\gamma}_{2 j-1}^{*}, \overline{\boldsymbol{\beta}}_{2 j-1}^{*}, \overline{\boldsymbol{\gamma}}_{2 j-1}^{*}$, which we call Boos-Jimbo-Miwa-Smirnov (BJMS) fermions. This construction is applied to solving the longstanding problem of computing the one-point functions for the sG model on the plane 2 and on the cylinder [3. To be precise, in addition to the BJMS fermions, certain fermionic screening operators are used to create the primary fields $\Phi_{\alpha+2 m \frac{1-\nu}{\nu}}$ and their descendants from $\Phi_{\alpha}$. We shall not discuss them as they will be irrelevant for the purpose of this paper. Let us emphasise one more time that the relation between the BJMS fermionic descendants and the Virasoro descendants can be computed. This has been done in the quotient space by the action of the local integrals of motion up to level 6 in [1] and on the level 8 in [28]. For studying the one-point functions it is sufficient to work in the quotient space. However, it is not quite sufficient for the goal of the present paper, we shall comment on this point soon.

Though the BBS fermions and the BJMS fermions have been introduced by different methods and for different reasons, there is a certain similarity between the two. This similarity motivated us to investigate the situation closely. On one hand we have the form factor formulae for the descendants written in terms of the BBS fermions. On the other hand we have local operators created by the BJMS fermions. Quite generally, if we insert such a local operator on the cylinder and take any eigenvectors of the Matsubara transfer-matrix as asymptotic conditions, then the partition function can be expressed in terms of a single function $\omega(\zeta, \xi)$. In particular, we can put an excited state to the left and the ground state to the right. Then the infinite volume limit in the Matsubara direction can be performed. It is clear that the result is nothing but a form factor. This provides us with the possibility for 
comparison. To our great surprise, the BBS and the BJMS fermions are completely equivalent. In the multi-index notation (see (11.19) ) the statement is this:

$$
\begin{aligned}
\text { If } & \mathcal{O}_{\alpha}=\boldsymbol{\beta}_{I^{+}}^{*} \overline{\boldsymbol{\beta}}_{\bar{I}^{+}}^{*} \bar{\gamma}_{\bar{I}^{-}}^{*} \boldsymbol{\gamma}_{I^{-}}^{*} \Phi_{\alpha}, \\
\text { then } & L_{\mathcal{O}_{\alpha}}^{(\star)}=\boldsymbol{\mu}^{\frac{1}{\nu}\left(\left|I^{-}\right|+\left|I^{+}\right|+\left|\bar{I}^{-}\right|+\left|\bar{I}^{+}\right|\right)} \psi_{I^{+}}^{*} \bar{\psi}_{\bar{I}^{+}}^{*} \bar{\chi}_{\bar{I}^{-}}^{*} \chi_{I^{-}}^{*} M_{0}^{(\star)},
\end{aligned}
$$

where $|I|$ denotes the sum of the entries of the multi-index $I$. To be precise we have to add some fermionic screening operators in (1.4) for $m \neq 0$, but in the infinite volume they are irrelevant as explained in Section 6. For us the precise identification (1.4)(1.5) came absolutely unexpected. It demonstrates a remarkable self-consistency of the sG model: taking two complicated problems, that of computing the form factors of descendants and that of computing the one-point functions on the cylinder (at finite temperature) and going to the very bottom of them we find the same fermionic structure.

The profit from the identification (1.4)-(1.5) is twofold.

First, since the BJMS descendants can be quantitatively related to the Virasoro descendants, the form factors of the latter can be computed. There is one technical obstacle here: up to now we were able to identify the BJMS and the Virasoro descendants only modulo the action of the local integrals of motion. This was quite sufficient for one-point functions, but for form factors we would like to have the complete answer. The technical problem which one needs to solve for this goal is explained at the end of Section 7.

Second, in [17] the null-vectors are found in terms of the BBS fermions. So, using (1.4)-(1.5) we can identify them for the BJMS fermions. To keep the present paper within a reasonable size, we leave the detailed study of the null-vectors to a separate publication. Here we shall consider only the chiral null-vectors for the field $\Phi_{(2 m-1) \frac{1-\nu}{\nu}}, m \geq 1$ which corresponds in the CFT language to $\Phi_{1,2 m}$. Let us give the simplest example which is the singular vector on level $2 m$. This singular vector is written as

$$
\boldsymbol{\beta}_{1}^{*} \boldsymbol{\gamma}_{2 m-1}^{*} \Phi_{1,2 m}
$$

At the moment comparison can be made only modulo the local integrals of motion, but even with this simplification the Virasoro counterpart of (1.6) looks really horrific. Using the formulae of [1, 28] we find perfect agreement up to level 8. The fermionic formulae are also simple for other null vectors which are the descendants of the singular vector in the Virasoro language. We think that this nice simplicity is another evidence of the universality of the fermionic picture.

The plan of the paper is as follows. In Section 2 we briefly review the form factors axioms in application to the sG model. In Section 3 we discuss certain integrals which play a basic role for the description of the $\mathrm{sG}$ form factors. In Section 4 we present the formulae for the form factors. Section 5 introduces the BBS fermions. In Section 6 we briefly discuss the BJMS fermions in the $\mathrm{SG}$ case. The origin of the fermionic description is the 6-vertex model as explained in Section 7. In Section 8 we discuss the infinite volume limit in the Matsubara direction. In Section 9 we present the main technical achievement of this paper, namely the computation 
of the function $\omega(\zeta, \xi)$ for infinite volume limit in the Matsubara direction. The equivalence of the BBS and the BJMS fermions is established in Section 10. The null vectors for $\Phi_{1,2 m}$ in terms of the BBS fermions are discussed in Section 11. Section 12 is devoted to comparison of these null vectors in the fermionic and the Virasoro descriptions.

\section{FORM FACTORS AXIOMS}

We are interested in form factors of the exponential fields $\Phi_{\alpha}(0)$ (1.2) and their descendants. In this paper we define form factors to be matrix elements of a local operator taken between excited states on the left and the vacuum state on the right, thus changing the convention of [7] where the opposite matrix elements were mostly studied. They are simply related by the crossing symmetry. We shall use the notation $\mathcal{O}_{\alpha}$ for descendants. These operators do not carry the topological charge, so, their form factors are non-trivial only in the case of an equal number of solitons and anti-solitons.

We do not consider the breather form factors since they can be obtained as residues of soliton ones. So, we have $2 n$ particles ( $n$ solitons and $n$ anti-solitons) with rapidities $\beta_{1}, \cdots \beta_{2 n}$. The form facotrs $f_{\mathcal{O}_{\alpha}}\left(\beta_{1}, \cdots, \beta_{2 n}\right)$ are meromorphic functions of these rapidities. They are vectors from the zero weight subspace of the space $\left(\mathbb{C}^{2}\right)^{\otimes 2 n}$. The standard basis in the $j$-th tensor component is denoted as $e_{j}^{ \pm}$.

The form factors are subject to three axioms. Formulating these axioms we follow the conventions of [7], namely, if two rapidities interchange we assume that the corresponding tensor components are permutated at the same time.

Symmetry axiom.

$$
\begin{aligned}
& S_{j, j+1}\left(\beta_{j}-\beta_{j+1}\right) f_{\mathcal{O}_{\alpha}}\left(\beta_{1}, \cdots, \beta_{j}, \beta_{j+1}, \cdots, \beta_{2 n}\right) \\
& =f_{\mathcal{O}_{\alpha}}\left(\beta_{1}, \cdots, \beta_{j+1}, \beta_{j}, \cdots, \beta_{2 n}\right)
\end{aligned}
$$

where $S_{i, j}$ is the soliton S-matrix [4]. Its explicit formula will be given later (10.3). Riemann-Hilbert problem axiom.

$$
f_{\mathcal{O}_{\alpha}}\left(\beta_{1}, \cdots, \beta_{2 n-1}, \beta_{2 n}+2 \pi i\right)=e^{-\frac{\pi i \nu}{1-\nu} \alpha \sigma_{2 n}^{3}} f_{\mathcal{O}_{\alpha}}\left(\beta_{2 n}, \beta_{1}, \cdots, \cdots, \beta_{2 n-1}\right) .
$$

Residue axiom.

$$
\begin{aligned}
& 2 \pi i \operatorname{res}_{\beta_{2 n}=\beta_{2 n-1}+\pi i} f_{\mathcal{O}_{\alpha}}\left(\beta_{1}, \cdots, \beta_{2 n-2}, \beta_{2 n-1}, \beta_{2 n}\right)= \\
& \left(1-e^{-\frac{\pi i \nu}{1-\nu} \alpha \sigma_{2 n}^{3}} S_{2 n-1,1}\left(\beta_{2 n-1}-\beta_{1}\right) \cdots S_{2 n-1,2 n-2}\left(\beta_{2 n-1}-\beta_{2 n-2}\right)\right) \\
& \times f_{\mathcal{O}_{\alpha}}\left(\beta_{1}, \cdots, \beta_{2 n-2}\right) \otimes s_{2 n-1,2 n}
\end{aligned}
$$

where $s_{i, j}=e_{i}^{+} \otimes e_{j}^{-}+e_{i}^{-} \otimes e_{j}^{+}$.

We change the standard basis of the tensor product to a new basis $w^{\epsilon_{1}, \cdots, \epsilon_{2 n}}\left(\beta_{1}, \cdots, \beta_{2 n}\right)$ described in [7], and express the form factors as

$$
f_{\mathcal{O}_{\alpha}}\left(\beta_{1}, \cdots, \beta_{2 n}\right)=Z\left(\beta_{1}, \cdots, \beta_{2 n}\right)
$$




$$
\times \sum_{\epsilon_{1}, \cdots, \epsilon_{2 n}= \pm} w^{\epsilon_{1}, \cdots, \epsilon_{2 n}}\left(\beta_{1}, \cdots, \beta_{2 n}\right) \frac{e^{\frac{\nu}{2(1-\nu)}\left(\sum_{p \in I^{-}} \beta_{p}-\sum_{p \in I^{+}} \beta_{p}+n \pi i\right)}}{\prod_{p \in I^{-}, q \in I^{+}} \sinh \frac{\nu}{1-\nu}\left(\beta_{p}-\beta_{q}\right)} \cdot \mathcal{F}_{\mathcal{O}_{\alpha}, n}\left(\beta_{I^{-}} \mid \beta_{I+}\right),
$$

where $I^{ \pm}=\left\{j \mid 1 \leq j \leq 2 n, \epsilon_{j}= \pm\right\}$, and the sum over $\epsilon_{j}$ 's is such that $\sharp\left(I^{+}\right)=$ $\sharp\left(I^{-}\right)$. We have introduced an overall multiplier

$$
Z\left(\beta_{1}, \cdots, \beta_{2 n}\right)=\frac{c^{n}}{n !} \prod_{i<j} \zeta\left(\beta_{i}-\beta_{j}\right) \cdot e^{\frac{1-2 \nu}{2(1-\nu)} n \sum_{j=1}^{2 n} \beta_{j}} .
$$

The formula for the function $\zeta(\beta)$ can be found in [7],

$$
c=\frac{\nu}{2(1-\nu) \pi^{2} \zeta(-\pi i)} .
$$

We have set also

$$
\beta_{I}=\left\{\beta_{i_{1}}, \cdots, \beta_{i_{n}}\right\}, \quad \text { if } \quad I=\left\{i_{1}, \cdots, i_{n}\right\} .
$$

We use this notation only for symmetric functions, so, the ordering of the indices $i_{1}, \cdots, i_{n}$ is irrelevant. The main property of the new basis and of the function $\zeta(\beta)$ is that

$$
\begin{aligned}
& \zeta\left(\beta_{i}-\beta_{i+1}\right) S_{i, i+1}\left(\beta_{i}-\beta_{i+1}\right) w^{\epsilon_{1}, \cdots, \epsilon_{i}, \epsilon_{i+1}, \cdots, \epsilon_{2 n}}\left(\beta_{1}, \cdots, \beta_{i}, \beta_{i+1}, \cdots, \beta_{2 n}\right) \\
& =\zeta\left(\beta_{i+1}-\beta_{i}\right) w^{\epsilon_{1}, \cdots, \epsilon_{i+1}, \epsilon_{i}, \cdots, \epsilon_{2 n}}\left(\beta_{1}, \cdots, \beta_{i+1}, \beta_{i}, \cdots, \beta_{2 n}\right) .
\end{aligned}
$$

Due to this property the first axiom is satisfied provided the essential part $\mathcal{F}_{\mathcal{O}_{\alpha}, n}\left(\beta_{I^{-}} \mid \beta_{I^{+}}\right)$ of the form factor is symmetric separately in $\beta_{I^{-}}$and $\beta_{I^{+}}$. It is well known that for this function one can write an integral representation. The integrals involved in this representation are quite remarkable, and we shall discuss them in detail in the next section.

Before closing this section a remark is in order concerning the phase in the right hand side of (2.2). With solitons are associated some quasi-local fields, which interpolate in- and out-states when time goes to $\mp \infty$. The phase in (2.2) specifies the locality property of a given field $\mathcal{O}_{\alpha}$ with respect to these interpolating fields. The BBS fermions which will be discussed in section 5 act on a primary field $\Phi_{\alpha}$ and create fields which share the same locality property (i.e. the same phase) as for $\Phi_{\alpha}$.

\section{INTEGRALS}

Consider the function

$$
\begin{aligned}
& \chi\left(\sigma \mid \beta_{1}, \cdots, \beta_{2 n}\right)=\prod_{j=1}^{2 n} \chi\left(\sigma, \beta_{j}\right), \\
& \chi(\sigma, \beta)=\frac{1}{2} e^{-\frac{1}{2(1-\nu)}\left(\sigma+\beta-\frac{\pi i}{2}\right)} \varphi\left(\sigma-\beta+\frac{\pi i}{2}\right),
\end{aligned}
$$

where $\varphi(\sigma)$ is defined in [7]. We shall not list explicitly the properties of $\varphi(\sigma)$, since they can be read from those of the function $\chi\left(\sigma \mid \beta_{1}, \cdots, \beta_{2 n}\right)$ which we are going to 
give. In what follows we use the symbols

$$
\begin{array}{r}
S=e^{\sigma}, \quad B_{j}=e^{\beta_{j}}, \quad Q=e^{\pi i \frac{1-\nu}{\nu}}, \quad A=e^{\pi i \alpha} \\
\mathfrak{s}=e^{\frac{2 \nu}{1-\nu} \sigma}, \quad \mathfrak{b}_{j}=e^{\frac{2 \nu}{1-\nu} \beta_{j}}, \quad \mathfrak{q}=e^{\pi i \frac{1}{1-\nu}}, \quad a=e^{\pi i \frac{\nu}{1-\nu} \alpha} .
\end{array}
$$

The function $\chi\left(\sigma \mid \beta_{1}, \cdots, \beta_{2 n}\right)$ is a meromorphic function of $\sigma$. For real $\beta_{j}$ 's it does not have singularities for $0>\operatorname{Im}(\sigma)>-\pi$. It has the following asymptotic behaviour for $\sigma \rightarrow \pm \infty$ :

$$
\begin{aligned}
& \chi\left(\sigma \mid \beta_{1}, \cdots, \beta_{2 n}\right) \simeq_{\sigma \rightarrow \infty} e^{-2 n \frac{1}{1-\nu} \sigma} x^{+}\left(\mathfrak{s} \mid \mathfrak{b}_{1}, \cdots, \mathfrak{b}_{2 n}\right) X^{+}\left(S \mid B_{1}, \cdots, B_{2 n}\right), \\
& \chi\left(\sigma \mid \beta_{1}, \cdots, \beta_{2 n}\right) \simeq \simeq_{\sigma \rightarrow-\infty} x^{-}\left(\mathfrak{s} \mid \mathfrak{b}_{1}, \cdots, \mathfrak{b}_{2 n}\right) X^{-}\left(S \mid B_{1}, \cdots, B_{2 n}\right),
\end{aligned}
$$

where

$$
\begin{aligned}
& x^{+}\left(\mathfrak{s} \mid \mathfrak{b}_{1}, \cdots, \mathfrak{b}_{2 n}\right)=1+\sum_{k=1}^{\infty} x_{k}^{+}\left(\mathfrak{b}_{1}, \cdots, \mathfrak{b}_{2 n}\right) \mathfrak{s}^{-k}, \\
& X^{+}\left(S \mid B_{1}, \cdots, B_{2 n}\right)=1+\sum_{k=1}^{\infty} X_{k}^{+}\left(B_{1}, \cdots, B_{2 n}\right) S^{-k}, \\
& x^{-}\left(\mathfrak{s} \mid \mathfrak{b}_{1}, \cdots, \mathfrak{b}_{2 n}\right)=\mathfrak{q}^{n} \prod_{j=1}^{2 n} \mathfrak{b}_{j}^{-\frac{1}{2}}\left(1+\sum_{k=1}^{\infty} x_{k}^{-}\left(\mathfrak{b}_{1}, \cdots, \mathfrak{b}_{2 n}\right) \mathfrak{s}^{k}\right), \\
& X^{-}\left(S \mid B_{1}, \cdots, B_{2 n}\right)=\prod_{j=1}^{2 n} B_{j}^{-1}\left(1+\sum_{k=1}^{\infty} X_{k}^{-}\left(B_{1}, \cdots, B_{2 n}\right) S^{k}\right) .
\end{aligned}
$$

The functions $x_{k}^{+}, X_{k}^{+}$(resp. $x_{k}^{-}, X_{k}^{-}$) are symmetric (resp. symmetric Laurent) polynomials of their arguments. They can be inductively computed from the functional equations

$$
\begin{aligned}
& \chi(\sigma+2 \pi i) p\left(\mathfrak{s q}^{4}\right)=\chi(\sigma) p\left(\mathfrak{s q}^{2}\right) \\
& \chi\left(\sigma+\frac{1-\nu}{\nu} \pi i\right) P(S Q)=\chi(\sigma) P(-S),
\end{aligned}
$$

where

$$
P(S)=\prod_{j=1}^{2 n}\left(S-B_{j}\right), \quad p(\mathfrak{s})=\prod_{j=1}^{2 n}\left(\mathfrak{s}-\mathfrak{b}_{j}\right) .
$$

The normalisation of $\chi(\sigma, \beta)$ is defined by the identity

$$
\chi(\sigma, \beta) \chi(\sigma, \beta+\pi i)=\frac{1}{(\mathfrak{s}-\mathfrak{b})\left(S^{2}-B^{2}\right)},
$$

which is convenient for computing the residues (2.3).

Consider now the integral

$$
I_{\alpha}\left(\beta_{1}, \cdots, \beta_{2 n}\right)=\int_{\mathbb{R}-i 0} \chi\left(\sigma \mid \beta_{1}, \cdots, \beta_{2 n}\right) e^{\frac{\nu \alpha}{1-\nu} \sigma} d \sigma .
$$


From the asymptotic behaviour of $\chi\left(\sigma \mid \beta_{1}, \cdots, \beta_{2 n}\right)$, it is clear that this integral converges for

$$
0<\operatorname{Re}(\alpha)<\frac{2 n}{\nu}
$$

In what follows we shall often omit the dependence of these functions on $\beta_{1}, \ldots, \beta_{2 n}$, abbreviating them to $I_{\alpha}, \chi(\sigma), x^{ \pm}(\mathfrak{s})$ and $X^{ \pm}(S)$.

We want to continue the integral $I_{\alpha}$ to the entire complex plane of $\alpha$. To this end for any $k \in \mathbb{Z}$ let us introduce Laurent polynomials of the form

$$
m^{(k)}(\mathfrak{s})=\sum_{j=-k+1}^{2 n-k} m_{j}^{(k)} \mathfrak{s}^{j}, \quad n^{(k)}(\mathfrak{s})= \begin{cases}\sum_{j=-k+1}^{0} n_{j}^{(k)} \mathfrak{s}^{j} & \text { if } k \geq 1 \\ \sum_{j=0}^{-k+1} n_{j}^{(k)} \mathfrak{s}^{j} & \text { if } k \leq 0\end{cases}
$$

which satisfy the identity

$$
p\left(\mathfrak{s q}^{-2}\right)=m^{(k)}(\mathfrak{s})+a^{-2} p(\mathfrak{s}) n^{(k)}\left(\mathfrak{s q}{ }^{-4}\right)-p\left(\mathfrak{s q}^{-2}\right) n^{(k)}(\mathfrak{s})
$$

where $p(\mathfrak{s})$ is defined in (3.3). For example

$$
\begin{aligned}
& n^{(1)}(\mathfrak{s})=\frac{1}{a^{-2} \mathfrak{q}^{4 n}-1} \\
& n^{(2)}(\mathfrak{s})=\frac{1}{a^{-2} \mathfrak{q}^{4 n}-1}\left(1+\frac{\mathfrak{q}^{4 n}\left(1-\mathfrak{q}^{2}\right)}{\left(a^{-2} \mathfrak{q}^{4 n+4}-1\right)} a^{-2} \sigma_{1}\left(\mathfrak{b}_{1}, \cdots, \mathfrak{b}_{2 n}\right) \mathfrak{s}^{-1}\right) .
\end{aligned}
$$

Here and after, $\sigma_{l}\left(\mathfrak{b}_{1}, \cdots \mathfrak{b}_{2 n}\right)$ stands for the $l$-th elementary symmetric polynomial in $\mathfrak{b}_{j}$ 's. Note that

$$
\begin{aligned}
\operatorname{res}_{a^{2}=q^{4 n}} n^{(1)}(\mathfrak{s}) \frac{d a^{2}}{a^{2}} & =-1, \\
\operatorname{res}_{a^{2}=q^{4 n+4}} n^{(2)}(\mathfrak{s}) \frac{d a^{2}}{a^{2}} & =-x_{1}^{+} \mathfrak{s}^{-1} .
\end{aligned}
$$

Using the functional equation (3.1) we can transform the integral defined in the original region (3.6) to

$$
I_{\alpha}=\int_{\mathbb{R}-i 0} \chi(\sigma) e^{\frac{\nu \alpha}{1-\nu} \sigma} \frac{m^{(1)}(\mathfrak{s})}{p\left(\mathfrak{s q} \mathfrak{q}^{-2}\right)} d \sigma+\int_{\Gamma} \chi(\sigma) e^{\frac{\nu \alpha}{1-\nu} \sigma} n^{(1)}(\mathfrak{s}) d \sigma,
$$

where the contour $\Gamma$ contains all the poles of the integrand in the strip $0>\operatorname{Im}(\sigma) \geq$ $-2 \pi$. It naturally splits into a sum of contours $\Gamma_{j}$ as is shown on fig. 1. 


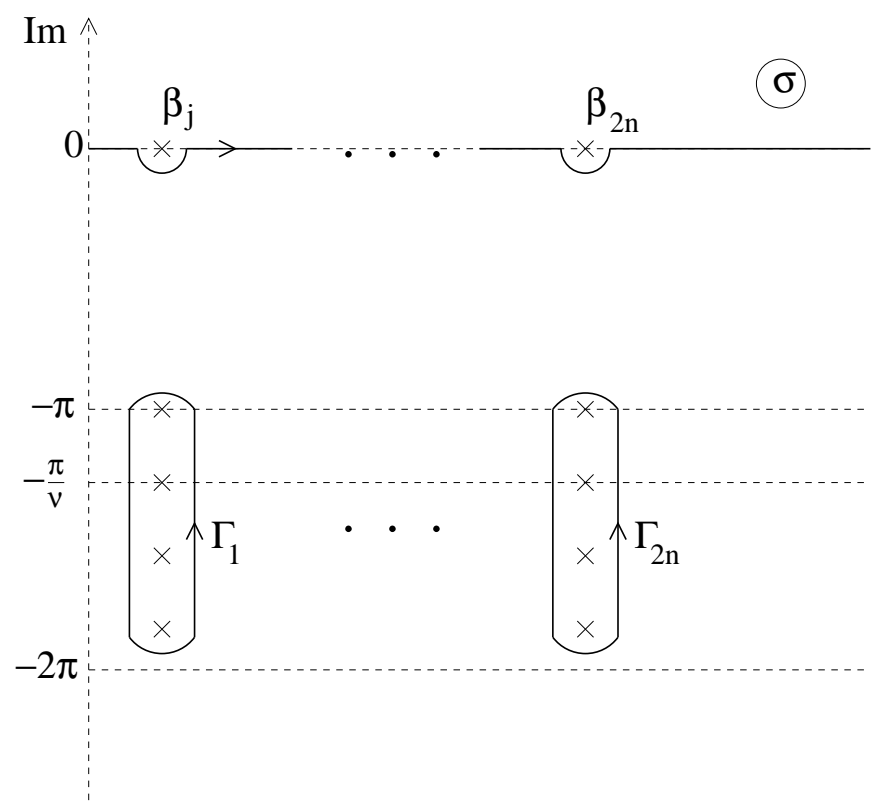

Fig.1: Contours for analytic continuation of $I_{\alpha}$.

The original contour $\Gamma$ is split into a sum of the contours $\Gamma_{j}$. Each $\Gamma_{j}$ surrounds a series of poles between the strip $-\pi \geq \operatorname{Im}(\sigma)>-2 \pi$ with the same real part as $\beta_{j}$.

The equivalence with the original definition is established by transforming $\Gamma$ to $(-\mathbb{R}-i 0) \cup(\mathbb{R}-2 \pi i-i 0)$. But the right hand side of (3.12) is analytical in $0<\operatorname{Re}(\alpha)<\frac{2 n}{\nu}+2$. So, we have managed to continue $I_{\alpha}$ analytically to the strip $\frac{2 n}{\nu} \leq \operatorname{Re}(\alpha)<\frac{2 n}{\nu}+2$. From the formula (3.8) we see that the result of the analytical continuation has simple poles at the points $2 n+(2 n+l) \frac{1-\nu}{\nu}$ with $l \geq 0$. We shall compute the residues at these poles later.

Let us proceed. Another representation for $I_{\alpha}$ is possible for $2<\operatorname{Re}(\alpha)<\frac{2 n}{\nu}+2$ :

$$
I_{\alpha}=\int_{\mathbb{R}-i 0} \chi(\sigma) e^{\frac{\nu \alpha}{1-\nu} \sigma} \frac{m^{(2)}(\mathfrak{s})}{p\left(\mathfrak{s q} \mathfrak{q}^{-2}\right)} d \sigma+\int_{\Gamma} \chi(\sigma) e^{\frac{\nu \alpha}{1-\nu} \sigma} n^{(2)}(\mathfrak{s}) d \sigma
$$

Indeed, the difference between (3.13) and (3.12) is

$$
\int_{\mathbb{R}-i 0} \chi(\sigma) e^{\frac{\nu \alpha}{1-\nu} \sigma} \frac{m^{(2)}(\mathfrak{s})-m^{(1)}(\mathfrak{s})}{p\left(\mathfrak{s q} \mathfrak{q}^{-2}\right)} d \sigma+\int_{\Gamma} \chi(\sigma) e^{\frac{\nu \alpha}{1-\nu} \sigma}\left(n^{(2)}(\mathfrak{s})-n^{(1)}(\mathfrak{s})\right) d \sigma .
$$

For $2<\operatorname{Re}(\alpha)<\frac{2 n}{\nu}+2$ the integrals are well defined. Moreover, by deforming the contour as before, it is easy to show that (3.14) is equal to zero. The right hand side of (3.13) is well defined in $2<\operatorname{Re}(\alpha)<\frac{2 n}{\nu}+4$. So, we have continued $I_{\alpha}$ into the strip $\frac{2 n}{\nu}+2 \leq \operatorname{Re}(\alpha)<\frac{2 n}{\nu}+4$. The series of poles at $2 n+(2 n+l) \frac{1-\nu}{\nu}$ continues, and new poles at $2(n+1)+2(n+l) \frac{1-\nu}{\nu}$ with $l \geq 0$ appear.

It is clear now how to continue $I_{\alpha}$ further. It is equally clear how to continue it to non-positive $\operatorname{Re}(\alpha)$. The final result is that $I_{\alpha}$ is a meromorphic function in $\mathbb{C}$ 
with simple poles at

$$
\alpha=2(n+m)+(2 n+l) \frac{1-\nu}{\nu}, \quad l, m \geq 0 ; \quad \alpha=-2 m-\frac{1-\nu}{\nu} l, \quad l, m \geq 0 .
$$

Let us compute the residues. Consider $I_{\alpha}$ in the strip $0<\operatorname{Re}(\alpha)<2+2 n \frac{1}{\nu}$. In this strip we have poles at the points $\alpha=2 n+(2 n+l) \frac{1-\nu}{\nu}, l \geq 0$, because at these points $m^{(1)}(\mathfrak{s})$ and $n^{(1)}(\mathfrak{s})$ have singularities. Obviously,

$$
\operatorname{res}_{\alpha=2 n+(2 n+l) \frac{1-\nu}{\nu}}\left(m^{(1)}(\mathfrak{s})+a^{-2} p(\mathfrak{s}) n^{(1)}\left(\mathfrak{s q}^{-4}\right)-p\left(\mathfrak{s q}{ }^{-2}\right) n^{(1)}(\mathfrak{s})\right)=0 .
$$

Then, using this equality with (3.10) in (3.12) and transforming the contour $\Gamma$ to $-(-(-\infty, \Lambda)) \cup(\Lambda-2 \pi i, \Lambda) \cup(-\infty-2 \pi i, \Lambda-2 \pi i)$ we find

$$
\operatorname{res}_{\alpha=2 n+(2 n+l) \frac{1-\nu}{\nu}} I_{\alpha} \frac{d a^{2}}{a^{2}}=-\lim _{\Lambda \rightarrow \infty} \int_{\Lambda-2 \pi i}^{\Lambda} \chi(\sigma) e^{2 n\left(1+\frac{\nu}{1-\nu}\right) \sigma} S^{l} d \sigma=-2 \pi i X_{l}^{+} .
$$

Next, consider $I_{\alpha}$ in the strip $2<\operatorname{Re}(\alpha)<4+2 n \frac{1}{\nu}$. Using (3.13), we compute the residues at the two series $\alpha=2 n+(2 n+l) \frac{1-\nu}{\nu}$ and $\alpha=2 n+2+(2 n+l) \frac{1-\nu}{\nu}$ of poles of $n^{(2)}(\mathfrak{s})$ given by (3.9) . For the former the result is the same because the term containing $\mathfrak{s}$ disappears in the limit $\Lambda \rightarrow \infty$. For the latter using (3.11) we obtain

$$
\operatorname{res}_{\alpha=2 n+2+(2 n+l) \frac{1-\nu}{\nu}} I_{\alpha} \frac{d a^{2}}{a^{2}}=-2 \pi i X_{l}^{+} x_{1}^{+} .
$$

Continuing along the same lines we come to the following result.

Proposition 3.1. The residues of $I_{\alpha}$ at $\alpha=2 n+2 m+(2 n+l) \frac{1-\nu}{\nu}$ for $l, m \geq 0$ are given by

$$
\underset{\alpha=2(n+m)+(2 n+l) \frac{1-\nu}{\nu}}{\operatorname{res}} I_{\alpha} d \alpha=-\frac{1-\nu}{\nu} \underset{S=\infty}{\operatorname{res}}\left(S^{l} X^{+}(S) \frac{d S}{S}\right) \underset{\mathfrak{s}=\infty}{\operatorname{res}}\left(\mathfrak{s}^{m} x^{+}(\mathfrak{s}) \frac{d \mathfrak{s}}{\mathfrak{s}}\right) .
$$

Similarly, the residues of $I_{\alpha}$ at $\alpha=-2 m-l \frac{1-\nu}{\nu}$ for $l, m \geq 0$ are given by

$$
\underset{\alpha=-2 m-l \frac{1-\nu}{\nu}}{\operatorname{res}} I_{\alpha} d \alpha=\frac{1-\nu}{\nu} \underset{S=0}{\operatorname{res}}\left(S^{-l} X^{-}(S) \frac{d S}{S}\right) \underset{\mathfrak{s}=0}{\operatorname{res}}\left(\mathfrak{s}^{-m} x^{-}(\mathfrak{s}) \frac{d \mathfrak{s}}{\mathfrak{s}}\right) .
$$

Now we give the main definition.

Definition 3.2. Consider two Laurent polynomials $\ell(\mathfrak{s})$ and $L(S)$. We define their pairing $(\ell, L)_{\alpha}$ by the following two requirements:

1. The pairing is bilinear.

2. If $\ell(\mathfrak{s})=\mathfrak{s}^{m}, L(S)=S^{l}$ then

$$
(\ell, L)_{\alpha}=I_{\alpha+2 m+\frac{1-\nu}{\nu} l} .
$$

The polynomials $\ell$ and $L$ are interpreted as cycles and forms in the classical limit. Actually there are two possibilities. In the limit $\nu \rightarrow 1$, $\ell$ describes cycles and $L$ describes one-forms. In the limit $\nu \rightarrow 0, \ell$ describes one-forms and $L$ describes cycles. The situation is very much dual, and there is no preferred choice to call one a cycle and the other a form. So we will call $\ell$ a $\mathfrak{q}$-deformed form and $L$ a $Q$-deformed form. The next two propositions describe exact forms. 
Proposition 3.3. For any Laurent polynomial $z(\mathfrak{s})$ we define

$$
\left.D_{a}[z](\mathfrak{s})=a^{-2} p(\mathfrak{s}) z(\mathfrak{s})-p(\mathfrak{s q})^{2}\right) z\left(\mathfrak{s q} \mathfrak{q}^{4}\right)
$$

and call it a $\mathfrak{q}$-exact form. For any Laurent polynomial $L(S)$ we have

$$
\left(D_{a}[z], L\right)_{\alpha}=0
$$

Proof. It is sufficient to consider the case $L(S)=S^{l}$. The procedure described above gives for any $\ell(\mathfrak{s})$

$$
(\ell, L)_{\alpha}=\int_{\mathbb{R}-i 0} \chi(\sigma) e^{\left(\frac{\alpha \nu}{1-\nu}+l\right) \sigma} \frac{m(\mathfrak{s})}{p\left(\mathfrak{s q}^{-2}\right)} d \sigma+\int_{\Gamma} \chi(\sigma) n(\mathfrak{s}) e^{\left(\frac{\alpha \nu}{1-\nu}+l\right) \sigma} d \sigma,
$$

where

$$
p\left(\mathfrak{s q} \mathfrak{q}^{-2}\right) \ell(\mathfrak{s})=m(\mathfrak{s})+a^{-2} p(\mathfrak{s}) n\left(\mathfrak{s q} \mathfrak{u}^{-4}\right)-p\left(\mathfrak{s q}^{-2}\right) n(\mathfrak{s})
$$

and $m(\mathfrak{s})$ is chosen in such a way that the integral in (3.20) converges. This is possible to do with a comfortable margin. If we take $\ell=D_{a}[z]$ it is easy to see that (3.21) is satisfied with

$$
m(\mathfrak{s})=0, \quad n(\mathfrak{s})=p\left(\mathfrak{s q ^ { 2 }}\right) z\left(\mathfrak{s q} \mathfrak{q}^{4}\right)
$$

Then the right hand side of (3.20) vanishes since $p\left(\mathfrak{s q}^{2}\right)$ cancels the singularities of $\chi(\sigma)$ inside $\Gamma$.

Proposition 3.4. For any Laurent polynomial $Z(S)$ we define

$$
D_{A}[Z](S)=Z(S) P(S)-A Z(S Q) P(-S)
$$

and call it a $Q$-exact form. For any Laurent polynomial $\ell(\mathfrak{s})$ we have

$$
\left(\ell, D_{A}[Z]\right)_{\alpha}=0
$$

Proof. It would be sufficient to consider a simple case when there is no need for regularisation of the integral, and then continue analytically. However, in what follows it is more instructive to have a direct proof for any $\alpha$. Figure 2 illustrates the proof. 


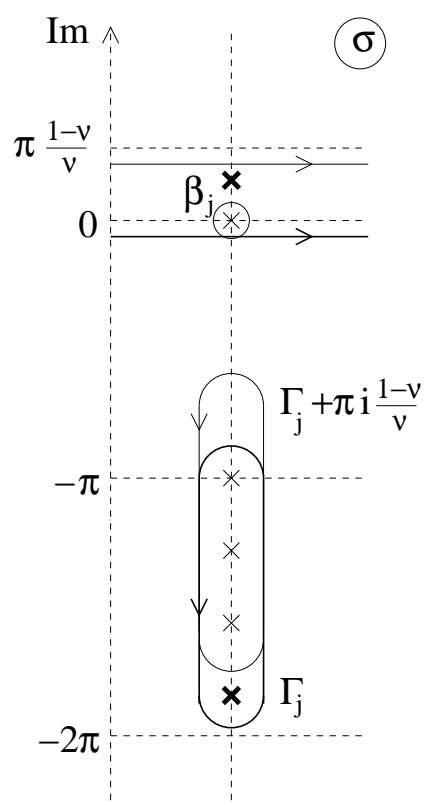

Fig.2: Possible poles arising in the proof of Proposition 3.4

Poles canceled by the factor $P(S)$ are represented by ordinary crosses inside circles. The residues at the remaining poles depicted by boldface crosses cancel each other.

From the definition we have

$$
\left(\ell, D_{A}[Z]\right)_{\alpha}=\int_{\mathbb{R}-i 0} \chi(\sigma) e^{\frac{\nu \alpha}{1-\nu} \sigma} D_{A}[Z](S) \frac{m(\mathfrak{s})}{\left.p(\mathfrak{s q})^{-2}\right)} d \sigma+\int_{\Gamma} \chi(\sigma) e^{\frac{\nu \alpha}{1-\nu} \sigma} D_{A}[Z](S) n(\mathfrak{s}) d \sigma,
$$

where $m(\mathfrak{s})$ and $n(\mathfrak{s})$ are polynomials satisfying (3.21) and are chosen by the requirement of convergence. Actually they may be different for different monomials in $Z(S)$, but this does not matter for the following computation.

Using the functional equation (3.2) one easily finds that

$$
\begin{aligned}
\left(\ell, D_{A}[Z]\right)_{\alpha} & =\left(\int_{\mathbb{R}-i 0}-\int_{\mathbb{R}+\pi i \frac{1-\nu}{\nu}-i 0}\right) \chi(\sigma) e^{\frac{\nu \alpha}{1-\nu} \sigma} P(S) Z(S) \frac{m(\mathfrak{s})}{p\left(\mathfrak{s q} \mathfrak{q}^{-2}\right)} d \sigma \\
& +\left(\int_{\Gamma}-\int_{\Gamma+\pi i \frac{1-\nu}{\nu}}\right) \chi(\sigma) e^{\frac{\nu \alpha}{1-\nu} \sigma} P(S) Z(S) n(\mathfrak{s}) d \sigma .
\end{aligned}
$$

Poles arise from $\chi(\sigma)$ and $1 / p\left(\mathfrak{s q}^{-2}\right)$. We ask which poles are inside the difference of contours $(\mathbb{R}-i 0)-\left(\mathbb{R}+\pi i \frac{1-\nu}{\nu}-i 0\right)$, or $\Gamma-\left(\Gamma+\pi i \frac{1-\nu}{\nu}\right)$. The poles of the first integrand at $\sigma=\beta_{j}$ are canceled by $P(S)$. So, the only remaining poles between $\mathbb{R}$ and $\mathbb{R}+\pi i \frac{1-\nu}{\nu}$ are at $\sigma=\beta_{j}+\pi i\left(1-\frac{1-\nu}{\nu}\left[\frac{\nu}{1-\nu}\right]\right)$. Similarly, the poles inside $\Gamma-\left(\Gamma+i \frac{1-\nu}{\nu}\right)$ are situated only at the points $\sigma=\beta_{j}-\pi i\left(1+\frac{1-\nu}{\nu}\left[\frac{\nu}{1-\nu}\right]\right)$. On fig. 2 the pole canceled by $P(S)$ is in the circle, and the remaining poles are fat. It 
follows from the functional equation (3.1) and the definition (3.21) that the residues at these remaining poles cancel each other.

The last definition which we would like to give here concerns the multiple integrals. Consider $k$ Laurent polynomials $\ell_{1}(\mathfrak{s}), \cdots \ell_{k}(\mathfrak{s})$ and $k$ Laurent polynomials $L_{1}(S), \cdots, L_{k}(S)$, and define the antisymmetric Laurent polynomials of $k$ variables:

$\ell^{(k)}\left(\mathfrak{s}_{1}, \cdots \mathfrak{s}_{k}\right)=\left(\ell_{1} \wedge \cdots \wedge \ell_{k}\right)\left(\mathfrak{s}_{1}, \cdots \mathfrak{s}_{k}\right), \quad L^{(k)}\left(S_{1}, \cdots, S_{k}\right)=\left(L_{1} \wedge \cdots \wedge L_{k}\right)\left(S_{1}, \cdots, S_{k}\right)$.

We define

$$
\left(\ell^{(k)}, L^{(k)}\right)_{\alpha}=\operatorname{det}\left(\left(\ell_{i}, L_{j}\right)_{\alpha}\right)_{i, j=1, \cdots, k}
$$

Then this pairing is generalised for all antisymmetric Laurent polynomials of $k$ variables $\ell^{(k)}, L^{(k)}$ by bilinearity.

\section{FormulaE FOR FORM FACTORS}

Now we are ready to write down the formulae for form factors (2.4). Let us first prepare a couple of symbols.

For a partition $I^{-} \sqcup I^{+}=\{1, \cdots, 2 n\}$ such that $\sharp\left(I^{-}\right)=\sharp\left(I^{+}\right)$, define the polynomials

$$
p_{I^{-}}(\mathfrak{s})=\prod_{j \in I^{-}}\left(\mathfrak{s}-\mathfrak{b}_{j}\right), \quad p_{I^{+}}(\mathfrak{s})=\prod_{j \in I^{+}}\left(\mathfrak{s}-\mathfrak{b}_{j}\right)
$$

so that we have $p(\mathfrak{s})=p_{I^{+}}(\mathfrak{s}) p_{I^{-}}(\mathfrak{s})$. We set also

$$
p_{I^{ \pm}, i}(\mathfrak{s})=\left[\mathfrak{s}^{i-n} p_{I^{ \pm}}(\mathfrak{s})\right]_{\geq},
$$

where []$_{\geq}$signifies the polynomial part. Essentially following [7] we define

$$
\begin{aligned}
& \ell_{I^{-} \sqcup I^{+}}^{(n)}\left(\mathfrak{s}_{1}, \cdots, \mathfrak{s}_{n}\right)=\left(\ell_{I^{-} \sqcup I^{+}, 0} \wedge \cdots \wedge \ell_{I^{-} \sqcup I^{+}, n-1}\right)\left(\mathfrak{s}_{1}, \cdots, \mathfrak{s}_{n}\right), \\
& \ell_{I^{-} \sqcup I^{+}, i}(\mathfrak{s})=a^{-1}\left\{p_{I^{-}}(\mathfrak{s})\left(p_{I^{+}, i}(\mathfrak{s})-p_{I^{+}, i}\left(\mathfrak{s q}^{2}\right)\right)\right. \\
& \left.\quad+\mathfrak{q}^{2(i-n)} p_{I^{+}}\left(\mathfrak{s q} \mathfrak{q}^{2}\right)\left(p_{I^{-}, i}(\mathfrak{s})-a^{2} p_{I^{-}, i}\left(\mathfrak{s q}^{2}\right)\right)\right\} .
\end{aligned}
$$

Formula (4.2) can also be rewritten as

$$
\begin{aligned}
& c_{I^{-} \sqcup I^{+}}(t, s):=\sum_{i=0}^{n-1}\left(\mathfrak{q}^{2} \mathfrak{t}\right)^{n-i} \ell_{I^{-} \sqcup I^{+}, i}(\mathfrak{s}) \\
& =\frac{a \mathfrak{t}}{\mathfrak{t}-\mathfrak{q}^{2} \mathfrak{s}} p\left(\mathfrak{q}^{2} \mathfrak{s}\right)-\frac{a^{-1} \mathfrak{q}^{2} \mathfrak{t}}{\mathfrak{q}^{2} \mathfrak{t}-\mathfrak{s}} p(\mathfrak{s}) \\
& +p_{I^{+}}\left(\mathfrak{q}^{2} \mathfrak{t}\right) p_{I^{-}}(\mathfrak{s})\left(\frac{a^{-1} \mathfrak{q}^{2} \mathfrak{t}}{\mathfrak{q}^{2} \mathfrak{t}-s}-\frac{a^{-1} \mathfrak{t}}{\mathfrak{t}-\mathfrak{s}}\right)+p_{I^{-}}(\mathfrak{t}) p_{I^{+}}\left(\mathfrak{q}^{2} \mathfrak{s}\right)\left(\frac{a^{-1} \mathfrak{t}}{\mathfrak{t}-\mathfrak{s}}-\frac{a \mathfrak{t}}{\mathfrak{t}-\mathfrak{q}^{2} \mathfrak{s}}\right)
\end{aligned}
$$

Now consider the ansatz (2.4). As it has already been mentioned, the symmetry axiom (2.1) is satisfied automatically if $\mathcal{F}_{\mathcal{O}_{\alpha}, n}\left(\beta_{I^{-}} \mid \beta_{I^{+}}\right)$is symmetric separately in 
$\beta_{I^{-}}$and in $\beta_{I^{+}}$. Furthermore, the Riemann-Hilbert problem axiom (2.2) is satisfied if we set

$$
\mathcal{F}_{\mathcal{O}_{\alpha}, n}\left(\beta_{I^{-}} \mid \beta_{I^{+}}\right)=e^{\frac{\nu}{2(1-\nu)}(1-\alpha)\left(\sum_{j=1}^{2 n} \beta_{j}-\pi i n\right)} \cdot\left(\ell_{I^{-} \sqcup I^{+}}^{(n)}, L^{(n)}\right)_{\alpha},
$$

where $L^{(n)}=L^{(n)}\left(S_{1}, \cdots, S_{n} \mid B_{1}, \cdots, B_{2 n}\right)$ is an arbitrary Laurent polynomial which is anti-symmetric in $S_{i}$ 's and symmetric in $B_{j}$ 's. This statement is proved by a direct computation similar to the one given in [7].

While these two axioms concern form factors with a fixed number of particles, the third, the residue axiom, relates the Laurent polynomials $L^{(n)}$ with different $n$. Using the procedure of computing the residues familiar from [7], one can reduce the residue axiom further to a simple system of recurrence relations given below (see (4.5)). For later use let us formulate it in a slightly more general setting.

Let $c$ be an integer, and consider a sequence of Laurent polynomials

$$
L^{(\star)}=\left\{L^{(l, n)}\left(S_{1}, \cdots, S_{l} \mid B_{1}, \cdots, B_{2 n}\right)\right\}_{\substack{l, n \geq 0 \\ l-n=c}}^{\substack{l \\ l-n=0}}
$$

which are anti-symmetric in $S_{i}$ 's and symmetric in $B_{j}$ 's. We refer to $L^{(l, n)}$ as the $2 n$-particle component of $L^{(\star)}$, and $c$ as the charge.

Definition 4.1. We say that $L^{(\star)}$ is a tower of charge $c$ if

$$
\begin{aligned}
& L^{(l, n)}\left(S_{1}, \cdots, S_{l-1}, B \mid B_{1}, \cdots, B_{2 n-2}, B,-B\right) \\
& =(-1)^{c} B \prod_{p=1}^{l-1}\left(B^{2}-S_{p}^{2}\right) \cdot L^{(l-1, n-1)}\left(S_{1}, \cdots, S_{l-1} \mid B_{1}, \cdots, B_{2 n-2}\right)
\end{aligned}
$$

holds for all $l, n \geq 1$ with $l-n=c$.

In the case $c=0$, we also write $L^{(n, n)}$ as $L^{(n)}$.

The statement is, the residue axiom is satisfied if the sequence $\left\{L^{(n)}\right\}_{n=0}^{\infty}$ in (4.4) is a tower of charge 0 . In other words, a tower of charge 0 , or more precisely its residue class modulo $Q$-exact forms, represents a descendant field $\mathcal{O}_{\alpha}$.

The most basic example of a tower of charge 0 is given by the polynomials which do not depend on the parameters $B_{1}, \cdots, B_{2 n}$ :

$$
M_{0}^{(n)}\left(S_{1}, \cdots, S_{n}\right)=\left\langle\Phi_{\alpha}\right\rangle \cdot S \wedge S^{3} \wedge \cdots \wedge S^{2 n-1} .
$$

This tower, denoted by $M_{0}^{(\star)}$, is identified with the primary field $\Phi_{\alpha}$, whose vacuum expectation value $\left\langle\Phi_{\alpha}\right\rangle$ has been found in [13].

\section{BBS FERMIONS.}

We are now in a position to introduce fermions which create towers out of the tower $M_{0}^{(\star)}$ for the primary field. In all formulas discussed below, we shall suppress the dependence on the parameters $B_{j}$. Since we deal with towers, however, their number $2 n$ can vary from place to place. For that reason, in this section alone, we indicate the $n$-dependence by a suffix, e.g., $P_{n}(S)=\prod_{j=1}^{2 n}\left(S-B_{j}\right)$. 
First we note that on the space of all towers there is an obvious action of the local integrals of motion. This is because the recursion relation (4.5) is unaffected by the multiplication $L^{(l, n)} \mapsto f(I, \bar{I}) L^{(l, n)}$, where $f(I, \bar{I})$ is an arbitrary polynomial in

$$
I_{2 j-1, n}=\sum_{k=1}^{2 n} B_{k}^{2 j-1}, \quad \bar{I}_{2 j-1, n}=\sum_{k=1}^{2 n} B_{k}^{-(2 j-1)} .
$$

In the construction of towers we shall fully make use of the freedom (5.1). We remark that the eigenvalues (5.1) arise in the expansion

$$
\sqrt{\frac{P_{n}(-Z)}{P_{n}(Z)}}= \begin{cases}e^{X_{n}(Z)} & (Z \rightarrow \infty) \\ e^{\bar{X}_{n}(Z)} & (Z \rightarrow 0)\end{cases}
$$

where

$$
X_{n}(Z)=\sum_{j=1}^{\infty} \frac{1}{2 j-1} Z^{-2 j+1} I_{2 j-1, n}, \quad \bar{X}_{n}(Z)=\sum_{j=1}^{\infty} \frac{1}{2 j-1} Z^{2 j-1} \bar{I}_{2 j-1, n}
$$

Now, following [17], let us introduce the polynomial

$$
C_{n}\left(S_{1}, S_{2}\right)=\frac{1}{4 \nu} S_{1} \sum_{\epsilon_{1}, \epsilon_{2}= \pm} \frac{P_{n}\left(\epsilon_{1} S_{1}\right) P_{n}\left(\epsilon_{2} S_{2}\right)}{\epsilon_{1} S_{1}+\epsilon_{2} S_{2}}
$$

where the overall coefficient is introduced for future convenience. This polynomial is characterised by the following requirements:

$$
\begin{aligned}
& C_{n}\left(S_{1}, S_{2}\right) \text { is odd in } S_{1} \text { and even in } S_{2}, \\
& \operatorname{deg}_{S_{1}} C_{n}\left(S_{1}, S_{2}\right)=2 n-1, \quad \operatorname{deg}_{S_{2}} C_{n}\left(S_{1}, S_{2}\right)=2 n-2, \\
& \left.C_{n}\left(S_{1}, S_{2}\right)\right|_{B_{2 n-1}=B, B_{2 n}=-B}=\left(S_{1}^{2}-B^{2}\right)\left(S_{2}^{2}-B^{2}\right) C_{n-1}\left(S_{1}, S_{2}\right),
\end{aligned}
$$

with $C_{1}\left(S_{1}, S_{2}\right)=-\frac{1}{\nu}\left(B_{1}+B_{2}\right) B_{1} B_{2} S_{1}$. We note also that the total homogeneous degree of $C_{n}\left(S_{1}, S_{2}\right)$ in $S_{i}$ 's and $B_{j}$ 's is $4 n$.

Due to the definition through the pairing (4.4), adding $Q$-exact forms to components of a tower $L^{(l, n)}$ does not change the form factors. A simple way of fixing this freedom is to restrict their degrees by

$$
0 \leq \operatorname{deg}_{S_{i}} L^{(l, n)}\left(S_{1}, \cdots, S_{l}\right) \leq 2 n-1 .
$$

If $\alpha$ is generic, any polynomial can be brought to this form by adding $Q$-exact forms.

Let $\mathcal{T}_{0, c}$ denote the space of all towers of charge $c$ satisfying (5.8). We introduce the action of fermions

$$
\psi_{0}^{*}(Z): \mathcal{T}_{0, c} \longrightarrow \mathcal{T}_{0, c+1}, \quad \chi_{0}^{*}(Z): \mathcal{T}_{0, c} \longrightarrow \mathcal{T}_{0, c-1}
$$


by defining

$$
\begin{aligned}
& \left(\psi_{0}^{*}(Z) L^{(\star)}\right)^{(l+1, n)}\left(S_{0}, \cdots, S_{l}\right) \\
& \quad=\frac{1}{P_{n}(-Z)} \frac{1}{l !} \operatorname{Skew}_{S_{0}, \cdots, S_{l}} C_{n}\left(Z, S_{0}\right) L^{(l, n)}\left(S_{1}, \cdots, S_{l}\right) \\
& \left(\chi_{0}^{*}(Z) L^{(\star)}\right)^{(l-1, n)}\left(S_{1}, \cdots, S_{l-1}\right) \\
& \quad=\frac{1}{P_{n}(-Z)} \frac{1}{2}\left(L^{(l, n)}\left(Z, S_{1}, \cdots, S_{l-1}\right)-L^{(l, n)}\left(-Z, S_{1}, \cdots, S_{l-1}\right)\right) .
\end{aligned}
$$

Here $L^{(\star)} \in \mathcal{T}_{0, c}$, and

$$
\text { Skew } f\left(x_{1}, \cdots, x_{l}\right)=\sum_{\sigma \in \mathfrak{S}_{l}} \operatorname{sgn} \sigma f\left(x_{\sigma(1)}, \cdots, x_{\sigma(l)}\right)
$$

stands for skew symmetrisation. Because of (5.6) and (5.7), $\psi_{0}^{*}(Z), \chi_{0}^{*}(Z)$ preserve the degree condition (5.8) and the recurrence relation (4.5). It is also easy to see that these operators mutually anticommute.

Choosing representatives of restricted degrees (5.8) is convenient to prove the completeness. Namely, following [18] it should be possible to prove that $\psi_{0}^{*}(Z)$, $\chi_{0}^{*}(Z)$ create the complete set of solutions to the recurrence relations over the ring of $I_{2 j-1}, \bar{I}_{2 j-1}$.

Now we are confronted with the task of identifying these towers with local operators. Notice that the coefficients entering the form factor axioms (2.1) $-(2.3)$ are periodic in $\alpha$ of period $2(1-\nu) / \nu$. Hence for all integers $m$ the axioms are the same for the fields $\Phi_{\alpha+2 m \frac{1-\nu}{\nu}}$ and their descendants. One has to be able to identify the towers corresponding to all these fields. Consider the simplest case: the tower of

$$
M_{m}^{(n)}=\left\langle\Phi_{\alpha+2 m \frac{1-\nu}{\nu}}\right\rangle \cdot S^{2 m+1} \wedge S^{2 m+2} \wedge \cdots \wedge S^{2 n+2 m-1} \prod_{j=1}^{2 n} B_{j}^{-m}
$$

obviously corresponds to the form factors of the primary field $\Phi_{\alpha+2 m \frac{1-\nu}{\nu}}(0)$. How does this fit into our description in terms of polynomials satisfying the restriction (5.8)? Obviously, for $m>0$ (resp. $m<0$ ) the Laurent polynomial $M_{m}^{(n)}$ contains degrees higher than $2 n-1$ (resp. lower than 0 ). They have to be reduced using $Q$-exact forms. We need some general description of this procedure which looks completely random for the moment.

The solution to the above problem was found in [17] by considering the classical limit. In the classical case the variables $S_{1}, \cdots, S_{n}$ turn into the separated variables. With our conventions the local classical observables are represented by polynomials $L_{\mathcal{O}_{\alpha}}^{(n)}$ of odd degrees in all $S_{j}$. So, the idea is to bring the towers created by $\psi_{0}^{*}(Z)$, $\chi_{0}^{*}(Z)$ to this form using $Q$-exact forms. We shall see that a very transparent structure will emerge.

In order to make the fermions odd in $Z$, it suffices to multiply them by (5.2) (that is, by making use of the integrals of motion). To make their action odd also in $S$, we modify $C_{n}\left(S_{1}, S_{2}\right)$ by adding $Q$-exact forms in $S_{2}$. Namely, we introduce formal 
series $C_{ \pm, n}\left(S_{1}, S_{2}\right)$ such that

$$
C_{ \pm, n}\left(S_{1}, S_{2}\right) \equiv C_{n}\left(S_{1}, S_{2}\right) \quad\left(S_{1}^{ \pm 1} \rightarrow \infty, \bmod Q \text {-exact forms in } S_{2}\right),
$$

and which are odd in both variables. This can be achieved by setting

$$
C_{ \pm, n}\left(S_{1}, S_{2}\right)=\frac{1}{2} \sum_{\epsilon_{1}, \epsilon_{2}= \pm} \epsilon_{1} \epsilon_{2} P_{n}\left(\epsilon_{1} S_{1}\right) P_{n}\left(\epsilon_{2} S_{2}\right) \tau_{ \pm}\left(\epsilon_{2} S_{2} / \epsilon_{1} S_{1}\right)
$$

where

$$
\begin{aligned}
& \tau_{+}(x)=-\sum_{l=0}^{\infty}(-x)^{l} \frac{i}{2 \nu} \cot \frac{\pi}{2}\left(\alpha+\frac{l}{\nu}\right), \\
& \tau_{-}(x)=\sum_{l=-\infty}^{-1}(-x)^{l} \frac{i}{2 \nu} \cot \frac{\pi}{2}\left(\alpha+\frac{l}{\nu}\right) .
\end{aligned}
$$

Expand $C_{ \pm, n}(Z, S)$ in $Z^{\mp 1}$. Their coefficients of $Z^{2 j-1}$ where $j<0$ or $j>n$ are $Q$-exact forms in $S$, and the remaining terms read as

$$
\begin{aligned}
& C_{+, n}(Z, S) \equiv \sum_{j=1}^{n} Z^{2 n-2 j+1} p_{2 j-1}(S) \quad(\bmod Q \text {-exact forms in } S), \\
& p_{2 j-1}(S)=\sum_{k=1}^{n+j} S^{2 k-1} p_{2 j-1,2 k-1}, \quad p_{2 j-1,2 n+2 j-1}=\frac{i}{\nu} \cdot \cot \frac{\pi}{2}\left(\alpha+\frac{2 j-1}{\nu}\right),
\end{aligned}
$$

$$
C_{-, n}(Z, S) \equiv \sum_{j=1}^{n} Z^{2 j-1} \bar{p}_{2 j-1}(S) \quad(\bmod Q \text {-exact forms in } S)
$$

$$
\bar{p}_{2 j-1}(S)=\sum_{k=1}^{n+j} S^{2 n-2 k+1} \bar{p}_{2 j-1,2 k-1} \quad \bar{p}_{2 j-1,2 n+2 j-1}=\sigma_{2 n}(B)^{2} \cdot \frac{i}{\nu} \cot \frac{\pi}{2}\left(\alpha-\frac{2 j-1}{\nu}\right) .
$$

After these modifications we define new fermions as formal series at $Z^{ \pm 1}=\infty$ :

$$
\begin{aligned}
& \left(\psi^{\prime *}(Z) L^{(\star)}\right)^{(l+1, n)}\left(S_{0}, \cdots, S_{l}\right) \\
& \quad=\frac{1}{\sqrt{P_{n}(Z) P_{n}(-Z)}} \frac{1}{l !} \operatorname{Skew}_{S_{0}, \cdots, S_{l}} C_{+, n}\left(Z, S_{0}\right) L^{(l, n)}\left(S_{1}, \cdots, S_{l}\right), \\
& \left(\bar{\psi}^{\prime} *(Z) L^{(\star)}\right)^{(l+1, n)}\left(S_{0}, \cdots, S_{l}\right) \\
& \quad=\frac{1}{\sqrt{P_{n}(Z) P_{n}(-Z)}} \frac{1}{l !} \operatorname{Skew}_{S_{0}, \cdots, S_{l}} C_{-, n}\left(Z, S_{0}\right) L^{(l, n)}\left(S_{1}, \cdots, S_{l}\right),
\end{aligned}
$$

where the right hand sides are understood as power series expansions in $Z^{\mp 1}$. Define also $\chi^{*}(Z), \bar{\chi}^{*}(Z)$ by the same formula (5.10) as for $\chi_{0}^{*}(Z)$, replacing

$$
\frac{1}{P_{n}(-Z)} \longrightarrow \frac{1}{\sqrt{P_{n}(Z) P_{n}(-Z)}}
$$

and Taylor expanding in $Z^{\mp 1}$. 
Finally we define $\psi^{*}(Z), \bar{\psi}^{*}(Z), \chi^{*}(Z), \bar{\chi}^{*}(Z)$ by a Bogolubov transform

$$
\begin{array}{ll}
\psi^{*}(Z)=e^{\Xi} \psi^{\prime *}(Z) e^{-\Xi}, & \bar{\psi}^{*}(Z)=e^{\Xi} \bar{\psi}^{\prime *}(Z) e^{-\Xi}, \\
\chi^{*}(Z)=e^{\Xi} \chi^{\prime *}(Z) e^{-\Xi}, & \bar{\chi}^{*}(Z)=e^{\Xi} \bar{\chi}^{*}(Z) e^{-\Xi},
\end{array}
$$

where $\Xi$ acting on the $2 n$-particle component is

$$
\begin{aligned}
& \Xi_{n}=\oint \oint \frac{d Z}{2 \pi i Z} \frac{d X}{2 \pi i X}\left(\widehat{C}_{+, n}(Z, X) \psi(Z) \bar{\chi}(X)+\widehat{C}_{-, n}(Z, X) \bar{\psi}(Z) \chi(X)\right), \\
& \widehat{C}_{ \pm, n}(Z, X)=\frac{1}{\sqrt{P_{n}(Z) P_{n}(-Z)} \sqrt{P_{n}(X) P_{n}(-X)}} C_{ \pm, n}(Z, X) .
\end{aligned}
$$

In the above we have introduced formally the annihilation operators $\psi(Z), \bar{\psi}(Z)$, $\chi(Z), \bar{\chi}(Z)$, which are canonically conjugate to $\psi^{\prime *}(Z), \bar{\psi}^{*}(Z), \chi^{*}(Z), \bar{\chi}^{*}(Z)$, respectively and annihilate $M_{0}^{(\star)}$. Obviously, the same operators are canonically conjugated to $\psi^{*}(Z), \bar{\psi}^{*}(Z), \chi^{*}(Z), \bar{\chi}^{*}(Z)$. That is why we did not put prime in the notation. Note, for example, that

$$
\psi^{*}(Z)=\psi^{*}(Z)-\oint \frac{d X}{2 \pi i X} \widehat{C}_{+, n}(Z, X) \bar{\chi}(X)
$$

The modification (5.19), (5.20) is useful for separating the role of the two chiralities, as it will be explained shortly.

With the definition of $\psi^{*}(Z), \bar{\psi}^{*}(Z), \chi^{*}(Z), \bar{\chi}^{*}(Z)$ given above, let us write down their action on the tower $M_{0}^{(\star)}$. Suppose $k-k^{\prime}=l-n$.

$$
\begin{aligned}
& \left(\psi^{*}\left(Z_{1}\right) \cdots \psi^{*}\left(Z_{p}\right) \bar{\psi}^{*}\left(Z_{p+1}\right) \cdots \bar{\psi}^{*}\left(Z_{k}\right)\right. \\
& \left.\quad \times \bar{\chi}^{*}\left(X_{k^{\prime}}\right) \cdots \bar{\chi}^{*}\left(X_{q+1}\right) \chi^{*}\left(X_{q}\right) \cdots \chi^{*}\left(X_{1}\right) M_{0}^{(\star)}\right)^{(l, n)}\left(S_{1}, \cdots, S_{l}\right) \\
& =\left\langle\Phi_{\alpha}\right\rangle(-)^{k k^{\prime}} \frac{1}{\prod_{j=1}^{k} \sqrt{P_{n}\left(Z_{j}\right) P_{n}\left(-Z_{j}\right)} \prod_{j=1}^{k^{\prime}} \sqrt{P_{n}\left(X_{j}\right) P_{n}\left(-X_{j}\right)}} \cdot\left|\begin{array}{cc}
\mathcal{A} & \mathcal{B} \\
\mathcal{C} & \mathcal{D}
\end{array}\right|,
\end{aligned}
$$

where $\mathcal{A}, \mathcal{B}, \mathcal{C}$ and $\mathcal{D}$ are respectively $k \times k^{\prime}, k \times l, n \times k^{\prime}$ and $n \times l$ matrices:

$$
\mathcal{A}=\left(\begin{array}{cccccc}
0 & \cdots & 0 & C_{+}\left(Z_{1}, X_{q+1}\right) & \cdots & C_{+}\left(Z_{1}, X_{k^{\prime}}\right) \\
\vdots & & \vdots & \vdots & & \vdots \\
0 & \cdots & 0 & C_{+}\left(Z_{p}, X_{q+1}\right) & \cdots & C_{+}\left(Z_{p}, X_{k^{\prime}}\right) \\
C_{-}\left(Z_{p+1}, X_{1}\right) & \cdots & C_{-}\left(Z_{p+1}, X_{q}\right) & 0 & \cdots & 0 \\
\vdots & & \vdots & \vdots & & \vdots \\
C_{-}\left(Z_{k}, X_{1}\right) & \cdots & C_{-}\left(Z_{k}, X_{q}\right) & 0 & \cdots & 0
\end{array}\right) .
$$




$$
\begin{gathered}
\mathcal{B}=\left(\begin{array}{ccc}
C_{+}\left(Z_{1}, S_{1}\right) & \cdots & C_{+}\left(Z_{1}, S_{l}\right) \\
\vdots & & \vdots \\
C_{+}\left(Z_{p}, S_{1}\right) & \cdots & C_{+}\left(Z_{p}, S_{l}\right) \\
C_{-}\left(Z_{p+1}, S_{1}\right) & \cdots & C_{-}\left(Z_{p+1}, S_{l}\right) \\
\vdots & & \vdots \\
C_{-}\left(Z_{k}, S_{1}\right) & \cdots & C_{-}\left(Z_{k}, S_{l}\right)
\end{array}\right) \\
\mathcal{C}=\left(\begin{array}{ccc}
X_{1} & \cdots & X_{k^{\prime}} \\
\vdots & & \vdots \\
X_{1}^{2 n-1} & \cdots & X_{k^{\prime}}^{2 n-1}
\end{array}\right), \quad \mathcal{D}=\left(\begin{array}{ccc}
S_{1} & \ldots & S_{l} \\
\vdots & & \vdots \\
S_{1}^{2 n-1} & \ldots & S_{l}^{2 n-1}
\end{array}\right) .
\end{gathered}
$$

The Fourier modes are introduced by

$$
\begin{array}{ll}
\psi^{*}(Z)=\sum_{j=1}^{\infty} Z^{-2 j+1} \psi_{2 j-1}^{*}, & \chi^{*}(X)=\sum_{j=1}^{\infty} X^{-2 j+1} \chi_{2 j-1}^{*}, \\
\bar{\psi}^{*}(Z)=\sum_{j=1}^{\infty} Z^{2 j-1} \bar{\psi}_{2 j-1}^{*}, & \bar{\chi}^{*}(X)=\sum_{j=1}^{\infty} X^{2 j-1} \bar{\chi}_{2 j-1}^{*} .
\end{array}
$$

A similar determinant formula is obtained for $\psi_{0}^{*}\left(Z_{1}\right) \cdots \psi_{0}^{*}\left(Z_{k}\right) \chi_{0}^{*}\left(X_{k^{\prime}}\right) \cdots \chi_{0}^{*}\left(X_{1}\right) M_{0}^{(\star)}$, for which the $\mathcal{A}$-part is 0 . For completeness we give the Fourier decomposition for the annihilation operators

$$
\begin{array}{lll}
\psi(Z) & =\sum_{j=1}^{\infty} Z^{2 j-1} \psi_{2 j-1}, & \bar{\psi}(Z)=\sum_{j=1}^{\infty} Z^{-2 j+1} \bar{\psi}_{2 j-1}, \\
\chi(Z)=\sum_{j=1}^{\infty} Z^{2 j-1} \chi_{2 j-1}, & \bar{\chi}(Z)=\sum_{j=1}^{\infty} Z^{-2 j+1} \bar{\chi}_{2 j-1},
\end{array}
$$

and the non-vanishing commutation relations

$$
\left[\psi_{2 j-1}, \psi_{2 k-1}^{*}\right]_{+}=\left[\chi_{2 j-1}, \chi_{2 k-1}^{*}\right]_{+}=\left[\bar{\psi}_{2 j-1}, \bar{\psi}_{2 k-1}^{*}\right]_{+}=\left[\bar{\chi}_{2 j-1}, \bar{\chi}_{2 k-1}^{*}\right]_{+}=\delta_{j, k} .
$$

Let us explain the formula (5.21) using physicist's terminology, choosing $k=k^{\prime}$ for simplicity. Suppose we create a new tower starting from the primary one by application of several operators $\psi_{2 j-1}^{*}, \chi_{2 j-1}^{*}, \bar{\psi}_{2 j-1}^{*}, \bar{\chi}_{2 j-1}^{*}$. Let us take $n \gg j$ for all indices $j$ of the operators involved. Then the polynomial $M_{0}^{(n)}\left(S_{1}, \cdots, S_{n}\right)=$ $\left\langle\Phi_{\alpha}\right\rangle S \wedge S^{3} \wedge \cdots \wedge S^{2 n-1}$ can be considered as Fermi zone with two ends situated at $S$ and $S^{2 n-1}$. Roughly speaking the operator $\chi_{2 j-1}^{*}$ creates a hole at $S^{2 n-2 j+1}, \bar{\chi}_{2 j-1}^{*}$ a hole at $S^{2 j-1}, \psi_{2 j-1}^{*}$ a particle at $S^{2 n+2 j-1}$, and $\bar{\psi}_{2 j-1}^{*}$ a particle at $S^{-2 j+1}$.

The tower for the primary field is represented by the pure wedge product (4.6). Those for the descendants are linear combinations of pure wedge products with symmetric Laurent polynomials in $B_{j}$ as coefficients. We discuss which pure wedge products appear in the linear combination. Suppose $a_{j}, b_{j} \in 2 \mathbb{Z}_{>0}-1$, and we pick up

$$
\left(\psi_{a_{1}}^{*} \cdots \psi_{a_{p}}^{*} \bar{\psi}_{a_{p+1}}^{*} \cdots \bar{\psi}_{a_{k}}^{*} \bar{\chi}_{b_{k}}^{*} \cdots \bar{\chi}_{b_{q+1}}^{*} \chi_{b_{q}}^{*} \cdots \chi_{b_{1}}^{*} M_{0}^{(\star)}\right)^{(n, n)}\left(S_{1}, \cdots, S_{n}\right)
$$


from (5.21) taking the coefficient of $\prod_{j=1}^{p} Z_{j}^{-a_{j}} \prod_{j=p+1}^{k} Z_{j}^{a_{j}} \prod_{j=1}^{q} X_{j}^{-b_{j}} \prod_{j=q+1}^{k} X_{j}^{b_{j}}$. We assume that $a_{1}>\ldots>a_{p}, a_{p+1}<\ldots<a_{k}, b_{1}<\ldots<b_{q}$ and $b_{q+1}>\ldots>b_{k}$. Moreover we assume that $n$ is large enough so that $2 n-b_{q}>b_{q+1}$, too. Note that $m=p-q$ is the excess of particles at the right end of the Fermi zone, and $-m$ is the excess of particles at the left end. We call $m$ a weight. A basic example of a tower of weight $m$ is (5.11).

The procedure of taking the coefficient is as follows. First we take the coefficient of $Z_{j}^{-a_{j}}(1 \leq j \leq p)$ and $Z_{j}^{a_{j}}(p+1 \leq j \leq k)$ in the first $k$ rows. Second we take the coefficient of $X_{j}^{-b_{j}}(1 \leq j \leq q)$ and $X_{j}^{b_{j}}(q+1 \leq j \leq k)$ in the first $k$ columns. For example, take $1 \leq j \leq p$ and consider the coefficient of $Z_{j}^{-a_{j}}$ in $C_{+, n}\left(Z_{j}, X\right) / \sqrt{P_{n}\left(Z_{j}\right) P_{n}\left(-Z_{j}\right)}$. We expand this for $Z_{j} \rightarrow \infty$. The leading term is obtained from the approximation

$$
\sqrt{P_{n}\left(Z_{j}\right) P_{n}\left(-Z_{j}\right)} \sim Z_{j}^{2 n}
$$

the coefficient being $p_{a_{j}}(X)$. The sub-leading terms are multiples of $p_{2 l-1}(X)$ where $2 l-1<a_{j}$. Therefore, if we consider a partial-ordering of pure wedge products by the natural ordering of $a_{j}$, the sub-leading terms has coefficients of lower order. It is the same for $p+1 \leq j \leq k$ and the column expansions. Collecting the coefficients in the leading order we obtain a matrix, whose $(\mathcal{A}, \mathcal{B})$ part is given by

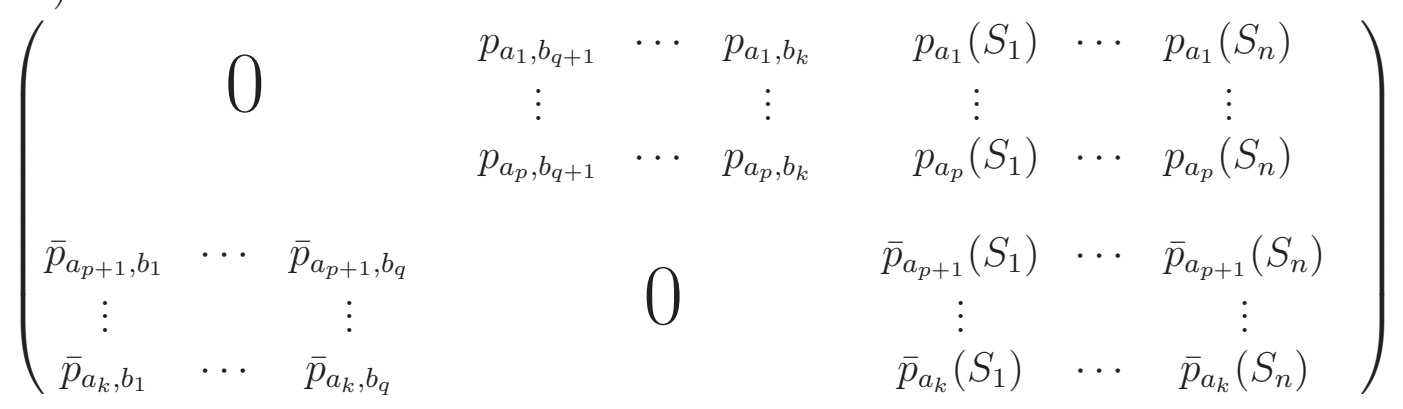

Consider $q+1 \leq j \leq k$ and set $b_{j}=2 l-1$. The $l$-th row in the $(\mathcal{C}, \mathcal{D})$ part gives rise to

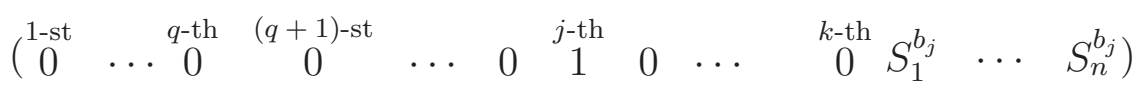

Similarly, for $1 \leq j \leq q$, set $2 n-b_{j}=2 l-1$. The $l$-th row in the $(\mathcal{C}, \mathcal{D})$ part gives rise to

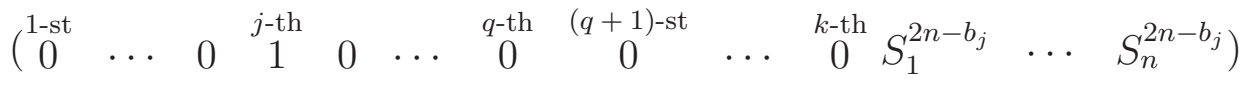

If $2 l-1 \notin\left\{b_{1}, \ldots, b_{q}, 2 n-b_{q+1}, \ldots, 2 n-b_{k}\right\}$ the $l$-th row in the $(\mathcal{C}, \mathcal{D})$ part gives rise to

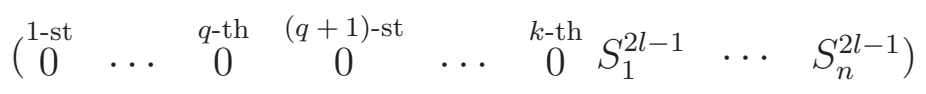


Subtracting certain multiples of rows (5.26), (5.27) from the $(\mathcal{A}, \mathcal{B})$ part (5.25), we change the latter to

$$
\left(\begin{array}{lll}
0 & 0 & \left(p_{a_{j}}\left(S_{k}\right)-\sum_{l=q+1}^{k} p_{a_{j}, b_{l}} S_{k}^{b_{l}}\right)_{\substack{j=1, \ldots, p \\
k=1, \ldots, n}} \\
0 & 0 & \left(\bar{p}_{a_{j}}\left(S_{k}\right)-\sum_{l=1}^{q} \bar{p}_{a_{j}, b_{l}} S_{k}^{2 n-b_{l}}\right)_{\substack{j=1, \ldots, p \\
k=1, \ldots, n}}
\end{array}\right)
$$

In physicist's terminology (5.26) implies the existence of a hole at $S^{b_{j}}$, and (5.27) hole at $S^{2 n-b_{j}}$. On the other hand we obtain a row from (5.28) by expanding it in the same power in $S_{k}$ :

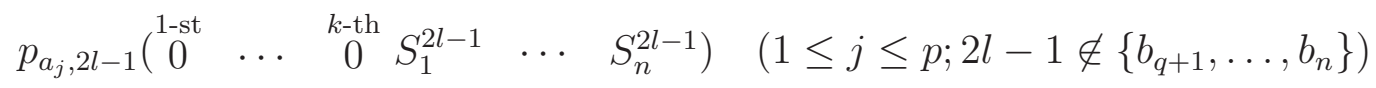

or

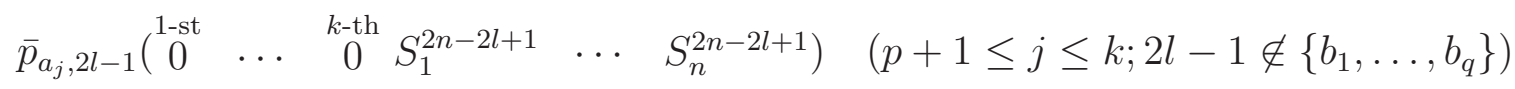

The former creates a particle at $S^{2 l-1}$ if $2 l-1>2 n$, and fills the hole at $S^{2 n-b_{j}}$ if $2 l-1=2 n-b_{j}$ for some $1 \leq j \leq q$. The latter creates a particle at $S^{2 n-2 l+1}$ if $2 l-1>2 n$, and fills the hole at $S^{b_{j}}$ if $2 n-2 l+1=b_{j}$ for some $q+1 \leq j \leq k$. It is important that the change caused by filling a hole by a particle occurs only if both particle and hole are near the right end, or both near the left end. Therefore, the weight $m$ is invariant by this change. In other words, one can define the weight $m$ of a tower without ambiguity. This is the effect of the Bogolubov transform (5.19), (5.20) which introduces the non-trivial block $\mathcal{A}$.

From (5.15), (5.16) it is easy to see that, up to a sign, the leading term in (5.24) is given by

$$
\begin{aligned}
\left\langle\Phi_{\alpha}\right\rangle\left(\frac{i}{\nu}\right)^{k} & \prod_{j=1}^{2 n} B_{j}^{q-p} \prod_{j=1}^{p} \cot \frac{\pi}{2}\left(\alpha+\frac{a_{j}}{\nu}\right) \prod_{j=p+1}^{k} \cot \frac{\pi}{2}\left(\alpha-\frac{a_{j}}{\nu}\right) \cdot S^{-a_{k}} \wedge \cdots \wedge S^{-a_{p+1}} \\
\wedge & \wedge \cdots\left(S^{b_{k}}\right)^{\vee} \cdots\left(S^{b_{q+1}}\right)^{\vee} \cdots\left(S^{2 n-b_{q}}\right)^{\vee} \cdots\left(S^{2 n-b_{1}}\right)^{\vee} \cdots \wedge S^{2 n-1} \\
& \wedge S^{2 n+a_{p}} \wedge \cdots \wedge S^{2 n+a_{1}} .
\end{aligned}
$$

Here $\left(S^{b}\right)^{\vee}$ represents a hole.

Our goal is to identify this fermionic picture with the content of local fields in the sG model. Here we do it qualitatively, leaving the quantitative identification to later sections. 
Returning to the primary fields we find

$$
\begin{aligned}
& \psi_{1}^{*} \cdots \psi_{2 m-1}^{*} \bar{\chi}_{2 m-1}^{*} \cdots \bar{\chi}_{1}^{*} M_{0}^{(\star)} \\
& =\frac{\left\langle\Phi_{\alpha}\right\rangle}{\left\langle\Phi_{\alpha+2 m \frac{1-\nu}{\nu}}\right\rangle} \cdot\left(\frac{i}{\nu}\right)^{m} \prod_{j=1}^{m} \cot \frac{\pi}{2 \nu}(\alpha \nu+(2 j-1)) M_{m}^{(\star)} \\
& \bar{\psi}_{1}^{*} \cdots \bar{\psi}_{2 m-1}^{*} \chi_{2 m-1}^{*} \cdots \chi_{1}^{*} M_{0}^{(\star)} \\
& =\frac{\left\langle\Phi_{\alpha}\right\rangle}{\left\langle\Phi_{\alpha-2 m \frac{1-\nu}{\nu}}\right\rangle} \cdot\left(\frac{i}{\nu}\right)^{m} \prod_{j=1}^{m} \cot \frac{\pi}{2 \nu}(\alpha \nu-(2 j-1)) M_{-m}^{(\star)}
\end{aligned}
$$

In general, it is now clear that the space of descendants of $M_{0}^{(\star)}$ by fermions, together with the action of the local integrals of motion, has the same character as that of the space of fields in CFT:

$$
\bigoplus_{m=-\infty}^{\infty} \nu_{\alpha+2 \frac{1-\nu}{\nu} m} \otimes \bar{\nu}_{\alpha+2 \frac{1-\nu}{\nu} m}
$$

where $\mathcal{V}_{\alpha}$ denotes the Virasoro Verma module with central charge $c=1-\frac{6 \nu^{2}}{1-\nu}$ and highest weight $\Delta_{\alpha}=\frac{\nu^{2}}{4(1-\nu)} \alpha(\alpha-2)$.

We remark that often it is convenient to change the definition slightly and work with fermions given in (5.21) wherein the block $\mathcal{B}$ is replaced by

$$
\mathcal{B} \rightarrow\left(\begin{array}{ccc}
C\left(Z_{1}, S_{1}\right) & \cdots & C\left(Z_{1}, S_{l}\right) \\
\vdots & & \vdots \\
C\left(Z_{k}, S_{1}\right) & \cdots & C\left(Z_{k}, S_{l}\right)
\end{array}\right) .
$$

The resulting fermions have mode expansions (5.22), (5.23) in odd degrees. Acting on the primary field, the Fourier components generate towers which coincide with (5.21) modulo $Q$-exact forms, but satisfying the restricted degree condition (5.8). It should be noted, however, that we cannot replace $C_{ \pm}$to $C$ in the block $\mathcal{A}$ because their second arguments are not the integration variables.

\section{BJMS FERMIONIC DESCRIPTION OF SG MODEL}

In the paper [1] we gave a fermionic description of CFT. This description has an advantage of being compatible with the integrable perturbation. As we explained in [2, 3] the logic of Perturbed Conformal Field Theory (PCFT) [8, 29] implies that in a generic situation ( $\nu$ and $\alpha$ irrational) there is a one-to-one correspondence between the local fields before and after perturbation, i.e. they consist of primary exponential fields $\Phi_{\alpha}$ and their descendants created by two copies of the Virasoro algebra with generators $\mathbf{l}_{k}, \overline{\mathbf{l}}_{k}$. Following [1] we consider the case

$$
0<\alpha<2 \text {. }
$$

Equivalence to the fermionic description implies that the local fields are created from the primary field by the local integrals of motion $\mathbf{i}_{2 k-1}, \overline{\mathbf{i}}_{2 k-1}$ and the fermions $\boldsymbol{\beta}_{2 k-1}^{*}, \boldsymbol{\gamma}_{2 k-1}^{*}, \overline{\boldsymbol{\beta}}_{2 k-1}^{*}, \overline{\boldsymbol{\gamma}}_{2 k-1}^{*}$. It is explained in [1, 28] how to recalculate the result of 
acting with the fermions in terms of the usual Virasoro descendants. Unfortunately, the techniques of [1, 28] allow us to do that only modulo the action of the local integrals of motion. At the end of the next section we give some explanation to this point. This was not a problem for the papers [2, 1] where the one-point functions in the sG model were calculated, because they vanish on the descendants created by the local integrals of motion. But in the context of the present paper this is an unpleasant restriction. We hope that the technical difficulties behind this problem will be resolved in the future. The origin of these difficulties will be explained in the next section. So, for the moment we work with the space

$$
\mathcal{V}_{\alpha}^{\text {quo }} \otimes \overline{\mathcal{V}}_{\alpha}^{\text {quo }}
$$

where

$$
\mathcal{V}_{\alpha}^{\text {quo }}=\mathcal{V}_{\alpha} / \sum_{k=1}^{\infty} \mathbf{i}_{2 k-1} \mathcal{V}_{\alpha}, \quad \overline{\mathcal{V}}_{\alpha}^{\text {quo }}=\overline{\mathcal{V}}_{\alpha} / \sum_{k=1}^{\infty} \overline{\mathbf{i}}_{2 k-1} \overline{\mathcal{V}}_{\alpha}
$$

Let us emphasise one more time that the operators $\mathbf{i}_{2 k-1}, \overline{\mathbf{i}}_{2 k-1}, \boldsymbol{\beta}_{2 k-1}^{*}, \boldsymbol{\gamma}_{2 k-1}^{*}, \overline{\boldsymbol{\beta}}_{2 k-1}^{*}$, $\bar{\gamma}_{2 k-1}^{*}$ are defined on $\mathcal{V}_{\alpha} \otimes \overline{\mathcal{V}}_{\alpha}$ and the restriction to $\mathcal{V}_{\alpha}^{\text {quo }} \otimes \overline{\mathcal{V}}_{\alpha}^{\text {quo }}$ is due to purely technical reasons.

To be precise the space $\mathcal{V}_{\alpha}^{\text {quo }} \otimes \overline{\mathcal{V}}_{\alpha}^{\text {quo }}$ allows a fermionic basis:

$$
\boldsymbol{\beta}_{I^{+}}^{*} \overline{\boldsymbol{\beta}}_{\bar{I}^{+}}^{*} \overline{\boldsymbol{\gamma}}_{\bar{I}^{-}}^{*} \boldsymbol{\gamma}_{I^{-}}^{*} \Phi_{\alpha}(0)
$$

where $\#\left(I^{+}\right)=\#\left(I^{-}\right), \#\left(\bar{I}^{+}\right)=\#\left(\bar{I}^{-}\right)$. We set generally

$$
\begin{aligned}
& \boldsymbol{\beta}_{I}^{*}=\boldsymbol{\beta}_{a_{1}}^{*} \cdots \boldsymbol{\beta}_{a_{p}}^{*}, \quad \boldsymbol{\gamma}_{I}^{*}=\boldsymbol{\gamma}_{a_{p}}^{*} \cdots \boldsymbol{\gamma}_{a_{1}}^{*}, \quad \overline{\boldsymbol{\beta}}_{I}^{*}=\overline{\boldsymbol{\beta}}_{a_{1}}^{*} \cdots \overline{\boldsymbol{\beta}}_{a_{p}}^{*}, \quad \overline{\boldsymbol{\gamma}}_{I}^{*}=\overline{\boldsymbol{\gamma}}_{a_{p}}^{*} \cdots \overline{\boldsymbol{\gamma}}_{a_{1}}^{*}, \\
& \text { for } I=\left\{a_{1}, \cdots, a_{p}\right\}, \quad a_{1}<a_{2}<\cdots<a_{p} .
\end{aligned}
$$

On the other hand the space $\mathcal{V}_{\alpha}^{\text {quo }} \otimes \overline{\mathcal{V}}_{\alpha}^{\text {quo }}$ can be realised as result of acting on the primary field $\Phi_{\alpha}$ by even generators of two chiral Virasoro algebras $\mathbf{l}_{-2 k}, \overline{\mathbf{l}}_{-2 k}$. The formulae relating the fermionic basis with the Virasoro basis can be found up to level 8 in [1, 28. The fermionic basis was used in [2, 3] because it allows one to compute the one-point functions.

We use the same convention for multi-indices as in [1, 3]:

$$
\begin{aligned}
\text { if } J & =\left\{j_{1}, \cdots, j_{p}\right\} \quad \text { then } \quad a J+b=\left\{a j_{1}+b, \cdots, a j_{p}+b\right\}, \\
I(m) & =\{1,2, \cdots, m\}, \quad I_{\text {odd }}(m)=2 I(m)-1 .
\end{aligned}
$$

The operators $\boldsymbol{\beta}_{2 k-1}^{*}, \boldsymbol{\gamma}_{2 k-1}^{*}$ and $\overline{\boldsymbol{\beta}}_{2 k-1}^{*}, \overline{\boldsymbol{\gamma}}_{2 k-1}^{*}$ are combined into the generating functions

$$
\begin{array}{ll}
\boldsymbol{\beta}^{*}(\lambda)=\sum_{k=1}^{\infty} \lambda^{-\frac{2 k-1}{\nu}} \boldsymbol{\beta}_{2 k-1}^{*}, & \boldsymbol{\gamma}^{*}(\lambda)=\sum_{k=1}^{\infty} \lambda^{-\frac{2 k-1}{\nu}} \boldsymbol{\gamma}_{2 k-1}^{*}, \\
\overline{\boldsymbol{\beta}}^{*}(\lambda)=\sum_{k=1}^{\infty} \lambda^{\frac{2 k-1}{\nu}} \overline{\boldsymbol{\beta}}_{2 k-1}^{*}, & \overline{\boldsymbol{\gamma}}^{*}(\lambda)=\sum_{k=1}^{\infty} \lambda^{\frac{2 k-1}{\nu}} \overline{\boldsymbol{\gamma}}_{2 k-1}^{*} .
\end{array}
$$

In a weak sense these series describe the asymptotics of holomorphic functions at $\lambda \rightarrow \infty$ and $\lambda \rightarrow 0$ for right and left chiralities respectively. When expanded at the opposite points, the same holomorphic functions create another interesting set of 
operators (actually a slight modification is needed [1, 3]). This allows us to define the operators

$$
\boldsymbol{\gamma}_{\text {screen }}^{*}(\lambda)=\sum_{k=1}^{\infty} \lambda^{2 k-\alpha} \boldsymbol{\gamma}_{\text {screen }, k}^{*}, \quad \overline{\boldsymbol{\beta}}_{\text {screen }}^{*}(\lambda)=\sum_{k=1}^{\infty} \lambda^{\alpha-2 k} \overline{\boldsymbol{\beta}}_{\text {screen }, k}^{*} .
$$

We define as usual

$$
\boldsymbol{\gamma}_{\text {screen }, I(m)}^{*}=\gamma_{\text {screen }, m}^{*} \cdots \gamma_{\text {screen }, 1}^{*}, \quad \overline{\boldsymbol{\beta}}_{\text {screen }, I(m)}^{*}=\overline{\boldsymbol{\beta}}_{\text {screen }, 1}^{*} \cdots \overline{\boldsymbol{\beta}}_{\text {screen }, m}^{*} .
$$

Then the $m$-fold screened primary field is by definition

$$
\Phi_{\alpha}^{(m)}(0)=i^{m} \boldsymbol{\mu}^{2 m} \prod_{j=1}^{m} \cot \frac{\pi \nu}{2}(2 j-\alpha) \times \overline{\boldsymbol{\beta}}_{\text {screen }, I(m)}^{*} \boldsymbol{\gamma}_{\text {screen }, I(m)}^{*} \Phi_{\alpha}(0),
$$

where the multiplier in the right hand side is introduced for convenience.

It is explained in [3] that $\mathcal{V}_{\alpha+2 m \frac{1-\nu}{\nu}}^{\text {quo }} \otimes \overline{\mathcal{V}}_{\alpha+2 m \frac{1-\nu}{\nu}}^{\text {quo }}$ for $m>0$ can be constructed as

$$
\begin{aligned}
& \boldsymbol{\beta}_{I^{+}}^{*} \overline{\boldsymbol{\beta}}_{I^{+}}^{*} \overline{\boldsymbol{\gamma}}_{\bar{I}^{-}}^{*} \boldsymbol{\gamma}_{I^{-}}^{*} \Phi_{\alpha+2 m \frac{1-\nu}{\nu}}(0) \\
& \cong C_{m}(\alpha) \boldsymbol{\beta}_{I^{+}+2 m}^{*} \overline{\boldsymbol{\beta}}_{\bar{I}^{+-2 m}}^{*} \overline{\boldsymbol{\gamma}}_{\bar{I}^{-}+2 m}^{*} \boldsymbol{\gamma}_{I^{-}-2 m}^{*} \boldsymbol{\beta}_{I_{\mathrm{odd}}(m)}^{*} \overline{\boldsymbol{\gamma}}_{I_{\mathrm{odd}}(m)}^{*} \Phi_{\alpha}^{(m)}(0),
\end{aligned}
$$

where for negative indices we set

$$
\boldsymbol{\gamma}_{-a}^{*}=-t_{a}(\alpha) \boldsymbol{\beta}_{a}, \quad \overline{\boldsymbol{\beta}}_{-a}^{*}=-t_{a}(2-\alpha) \overline{\boldsymbol{\gamma}}_{a}, \quad t_{a}(\alpha)=\frac{i}{\nu} \cot \frac{\pi}{2 \nu}(\alpha \nu+a),
$$

the only non-trivial anticommutation relations are

$$
\left[\boldsymbol{\beta}_{a}, \boldsymbol{\beta}_{b}^{*}\right]_{+}=\delta_{a, b}, \quad\left[\overline{\boldsymbol{\gamma}}_{a}, \overline{\boldsymbol{\gamma}}_{b}^{*}\right]_{+}=\delta_{a, b},
$$

and

$$
\boldsymbol{\beta}_{a} \Phi_{\alpha}^{(m)}(0)=0, \quad \bar{\gamma}_{a} \Phi_{\alpha}^{(m)}(0)=0
$$

In particular,

$$
\Phi_{\alpha+2 m \frac{1-\nu}{\nu}}(0) \cong C_{m}(\alpha) \boldsymbol{\beta}_{I_{\text {odd }}(m)}^{*} \bar{\gamma}_{I_{\text {odd }}(m)}^{*} \Phi_{\alpha}^{(m)}(0)
$$

where (see [3])

$$
C_{m}(\alpha)=\frac{\left\langle\Phi_{\alpha+m \frac{1-\nu}{\nu}}\right\rangle}{\left\langle\Phi_{\alpha}\right\rangle}(-i \nu)^{m} \prod_{j=1}^{m} \tan \frac{\pi}{2 \nu}(\alpha \nu+2 j-1) .
$$

The fermionic screening operators $\overline{\boldsymbol{\beta}}_{\text {screen }, 2 j-1}^{*}, \boldsymbol{\gamma}_{\mathrm{screen}, 2 j-1}^{*}$ play an important role in the CFT computations. However, in the $\mathrm{sG}$ case when we consider the one-point function on the cylinder [3] their contribution completely factors out. This results in the possibility of the identification in the weak sense:

$$
\Phi_{\alpha}^{(m)}(0) \stackrel{\mathrm{w}}{=} \Phi_{\alpha}(0) .
$$

This is a general fact for the sG model. We shall comment on it later.

Considering the formulae (6.8), (6.9), (6.10) we find an amazing similarity to the formula (5.11). This similarity was one of starting points for our present research. 
Recall that up to now we considered only the case $0<\alpha<2$. According to (6.6) it is actually sufficient to consider the fundamental domain

$$
0<\alpha<2 \frac{1-\nu}{\nu} \text {. }
$$

We excluded the point $\alpha=2 \frac{1-\nu}{\nu}$ from the fundamental domain for the following reason. An important role in the definition of the BJMS fermions is played by the function $\Delta_{\zeta}^{-1} \psi_{0}(\zeta, \alpha)$ discussed in the next section and its asymptotics (17.18). For $\alpha=2 \frac{1-\nu}{\nu}$ the asymptotic formula at $\zeta \rightarrow 0$ breaks down, and a logarithmic term appears from the $j=2$ term in the first sum and the $j=1$ term in the second sum. So, the case $\alpha=2 \frac{1-\nu}{\nu}$ requires a special treatment.

\section{SiX-VERTEX MODEL AS ORIGIN OF BJMS FERMIONIC DESCRIPTION}

We introduce fermions for $c<1 \mathrm{CFT}$ and for the $\mathrm{sG}$ model taking the scaling limit of the homogeneous and inhomogeneous six-vertex model on the cylinder. This is explained in details in the paper [3], so, we shall be very brief here. But we would like to repeat some facts from the paper [25] which is the cornerstone for all of our recent researches.

Let us consider the inhomogeneous six-vertex model on the cylinder. We count the sites in the space direction by indices $j$, and in the Matsubara direction by $\mathbf{m}$. The model can be inhomogeneous in both directions with the parameters of inhomogeneity $\zeta_{j}$ and $\tau_{\mathbf{m}}$. The Boltzmann weights are combined into the $L$-operator $L_{j, \mathbf{m}}\left(\zeta_{j} / \tau_{\mathbf{m}}\right)$. Using the equivalent XXZ spin chain language we introduce the operator $q^{2 \alpha S(0)} \mathcal{O}$ with $\mathcal{O}$ being local and $S(k)=\frac{1}{2} \sum_{j=-\infty}^{k} \sigma_{j}^{3}$. Then we consider the partition function as in the following figure:

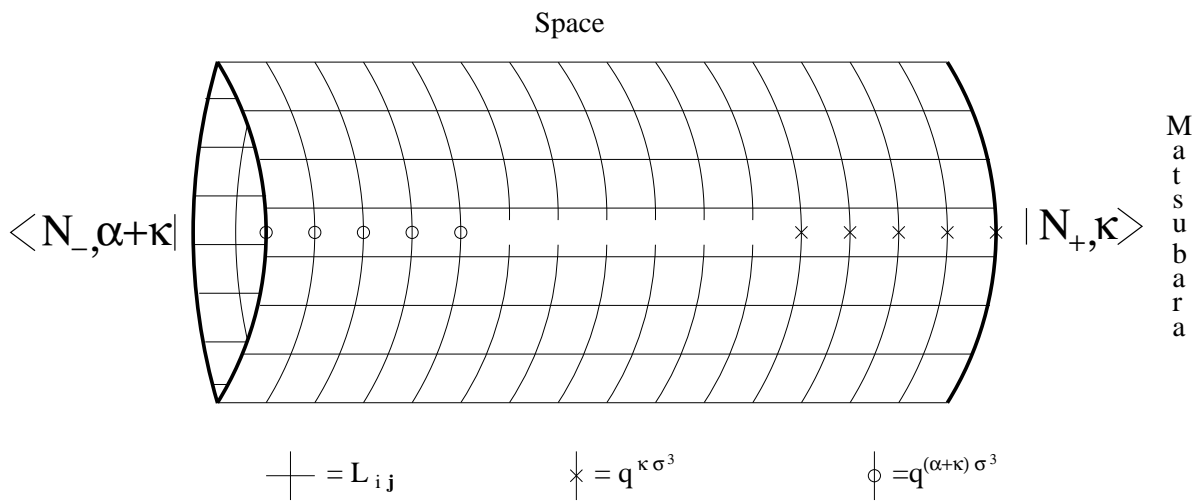

Fig.3: Partition function of the six-vertex model on a cylinder.

To each site of the lattice is attached a local Boltzmann weight $L_{j, \mathbf{m}}$. On one horizontal line a background field $q^{\kappa \sigma^{3}}$ is applied. On the same line, one allows in addition a local dislocation in the middle accompanied by an extra field $q^{\alpha \sigma^{3}}$ extending to the left, representing the insertion of a quasi-local operator $q^{2 \alpha S(0)} \mathcal{O}$.

The number of sites in the Matsubara direction is denoted by $\mathbf{n}$. The boundary conditions in the space direction are given by the eigenvectors $\left\langle N_{-}, \kappa+\alpha\right|$ and $\left|N_{+}, \kappa\right\rangle$ of the Matsubara transfer-matrices with twists $\kappa+\alpha$ and $\kappa$, and $N_{ \pm}$are 
the labels for different eigenvectors. In this formulation the number of sites in the space direction is not important as far as the operator $\mathcal{O}$ fits into the picture fig.3. The same computation can be used in a different situation. Put arbitrary boundary conditions on the right end of the cylinder, and allow it to grow to the right infinitely. Then the partition function will look for the eigenvector $|0, \kappa\rangle$ with the largest eigenvalue of the Matsubara transfer-matrix (ground state). Certainly one should not be extremely unlucky which means that the vector representing the boundary conditions should not be orthogonal to the ground state.

The partition function fig. 3 is written as

$$
\left\langle N_{-}, \kappa+\alpha\left|\operatorname{Tr}_{\mathrm{S}}\left(T_{\mathrm{S}, \mathbf{M}} q^{2 \kappa S+2 \alpha S(0)} \mathcal{O}\right)\right| N_{+}, \kappa\right\rangle,
$$

where $\mathbf{S}$ stands for space, $\mathbf{M}$ stands for Matsubara, $S$ is the total spin in the space direction, $T_{\mathrm{S}, \mathrm{M}}$ is the rectangular monodromy matrix in the tensor product of the space and the Matsubara Hilbert spaces.

Let us make a digression. We discuss a connection to form factors. Consider two quasi-local fields with opposite twists, $q^{-2 \alpha S(0)} \mathcal{O}_{1}$ and $q^{2 \alpha S(n)} \mathcal{O}_{2}$. For simplicity we take $\kappa=0$ and denote corresponding ground state by $\mid$ vac $\rangle$. In Section 8 we will see that in the infinite volume limit in the Matsubara direction, the $\kappa$-dependence is dropped. In order to compute the correlation function

$$
\left\langle\operatorname{vac}\left|\operatorname{Tr}_{\mathrm{S}}\left(T_{\mathrm{S}, \mathbf{M}} q^{-2 \alpha S(0)} \mathcal{O}_{1} q^{2 \alpha S(n)} \mathcal{O}_{2}\right)\right| \operatorname{vac}\right\rangle
$$

we have to glue two partition functions of this kind and sum over the intermediate states as in fig.4:

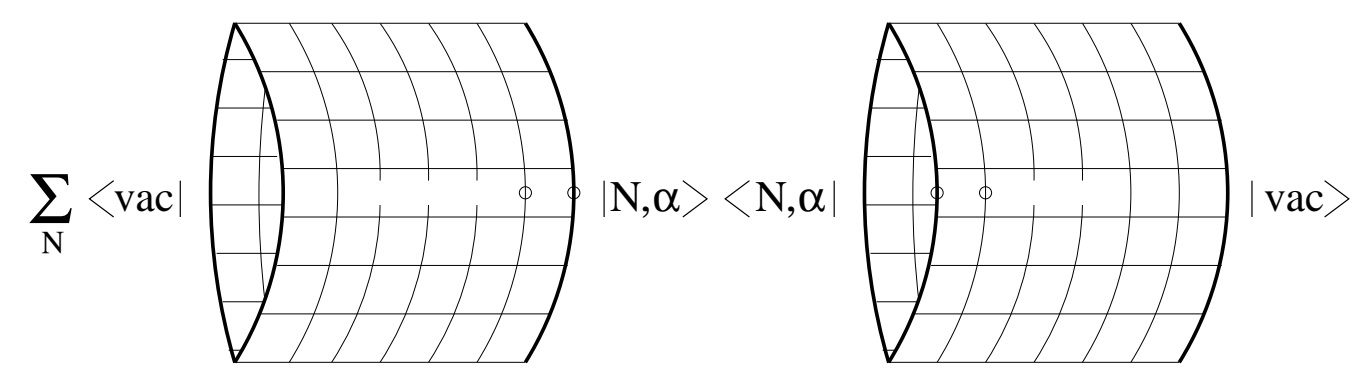

Fig.4: Form factor decomposition of the two-point function on the lattice.

This is nothing but the form factor decomposition in the Matsubara direction. We will come back to this subject in Section 9.

In the paper [25] we computed the functional

$$
Z_{\mathbf{n}}^{\kappa}\left\{q^{2 \kappa S+2 \alpha S(0)} \mathcal{O}\right\}=\frac{\left\langle N_{-}, \kappa+\alpha\left|\operatorname{Tr}_{S}\left(T_{S, \mathbf{M}} q^{2 \kappa S+2 \alpha S(0)} \mathcal{O}\right)\right| N_{+}, \kappa\right\rangle}{\left\langle N_{-}, \kappa+\alpha\left|\operatorname{Tr}_{S}\left(T_{S, \mathbf{M}} q^{2 \kappa S+2 \alpha S(0)}\right)\right| N_{+}, \kappa\right\rangle} .
$$

The dependence of $Z_{\mathbf{n}}^{\kappa}$ on the particular eigenvectors of the Matsubara transfermatrices is not explicitly exhibited. Obviously (7.1) is not a form factor but rather a ratio of the form factor of a descendant field to the form factor of the primary field. This should be remembered. 
The key objects to the computation of $Z_{\mathbf{n}}^{\kappa}$ were introduced in the paper [21]. In this paper it was shown that the quasi-local operators $q^{2 \alpha S(0)} \mathcal{O}$ can be created from the primary field by action of one bosonic and two fermionic operators: $\mathbf{t}^{*}(\zeta), \mathbf{b}^{*}(\zeta)$, $\mathbf{c}^{*}(\zeta)$. More precisely in the homogeneous case the quasi-local operators $q^{2 \alpha S(0)} \mathcal{O}$ are created by $\mathbf{t}_{p}^{*}, \mathbf{b}_{p}^{*}, \mathbf{c}_{p}^{*}$ defined through

$$
\mathbf{t}^{*}(\zeta)=\sum_{p=1}^{\infty}\left(\zeta^{2}-1\right)^{p-1} \mathbf{t}_{p}^{*}, \quad \mathbf{b}^{*}(\zeta)=\sum_{p=1}^{\infty}\left(\zeta^{2}-1\right)^{p-1} \mathbf{b}_{p}^{*}, \quad \mathbf{c}^{*}(\zeta)=\sum_{p=1}^{\infty}\left(\zeta^{2}-1\right)^{p-1} \mathbf{c}_{p}^{*}
$$

In particular $\mathbf{t}_{1}^{*} / 2$ is the right shift by one site along the lattice:

$$
\frac{1}{2} \mathbf{t}_{1}^{*}\left(q^{2 \alpha S(0)}\right)=q^{2 \alpha S(1)} .
$$

The completeness of this basis is proved in [30].

The main theorem [25] states that

$$
\begin{aligned}
& Z_{\mathbf{n}}^{\kappa}\left\{\mathbf{t}^{*}\left(\eta_{1}\right) \cdots \mathbf{t}^{*}\left(\eta_{m}\right) \mathbf{b}^{*}\left(\zeta_{1}\right) \cdots \mathbf{b}^{*}\left(\zeta_{k}\right) \mathbf{c}^{*}\left(\xi_{k}\right) \cdots \mathbf{c}^{*}\left(\xi_{1}\right)\left(q^{2 \alpha S(0)}\right)\right\} \\
& =\prod_{j=1}^{m} 2 \rho\left(\eta_{j}\right) \cdot \operatorname{det}\left(\omega\left(\zeta_{i}, \xi_{j}\right)\right)_{i, j=1, \cdots k}
\end{aligned}
$$

where

$$
\rho(\zeta)=\frac{T_{\mathrm{L}}(\zeta)}{T_{\mathrm{R}}(\zeta)}
$$

and $T_{\mathrm{L}}(\zeta)$ and $T_{\mathrm{R}}(\zeta)$ are respectively the eigenvalues of the left (with twist $\kappa+\alpha$ ) and the right (with twist $\kappa$ ) Matsubara transfer-matrices on the eigenvectors $\left\langle N_{-}, \kappa+\alpha\right|$ and $\left|N_{+}, \kappa\right\rangle$. The function $\omega(\zeta, \xi)$ was defined in [25] through a number of conditions as a quantum deformation of the normalised second kind differential on a hyperelliptic Riemann surface. For computational purposes it is useful to consider an equivalent, alternative formula [26] which is explained below.

Consider the eigenvector $\left|N_{+}, \kappa\right\rangle$. The corresponding eigenvalue is defined by the function $\mathfrak{a}(\zeta)$ which satisfies the DDV equation

$$
\log \mathfrak{a}(\zeta)=-2 \pi i \nu \kappa+\log \left(\frac{d(\zeta)}{a(\zeta)}\right)-\int_{\gamma} K(\zeta / \xi) \log (1+\mathfrak{a}(\xi)) \frac{d \xi^{2}}{\xi^{2}}
$$

where the cycle $\gamma$ goes around the zeros of the eigenvalue of the Baxter operator $Q_{\mathrm{R}}(\zeta)$ (Bethe roots) in the clockwise direction, as opposed to all other contours. The functions $a(\zeta)$ and $d(\zeta)$ are defined by using inhomogeneous parameters in the Matsubara direction. The formulae for them can be found in [25. We would like to emphasise that $\mathfrak{a}(\xi)$ depends only on the eigenvalue of the right transfer-matrix.

For completeness let us write the Baxter equation, and the definition of $\mathfrak{a}(\zeta)$

$$
T_{\mathrm{R}}(\zeta) Q_{\mathrm{R}}(\zeta)=d(\zeta) Q_{\mathrm{R}}(\zeta q)+a(\zeta) Q_{\mathrm{R}}\left(\zeta q^{-1}\right), \quad \mathfrak{a}(\zeta)=\frac{d(\zeta) Q_{\mathrm{R}}(\zeta q)}{a(\zeta) Q_{\mathrm{R}}\left(\zeta q^{-1}\right)} .
$$


Recall also that $\zeta^{\kappa} Q_{\mathrm{R}}(\zeta)$ is a polynomial in $\zeta^{2}$. The kernel in the integral equation is defined through a more general one

$$
K(\zeta)=K(\zeta, 0), \quad K(\zeta, \alpha)=\frac{1}{2 \pi i} \Delta_{\zeta} \psi_{0}(\zeta, \alpha)
$$

where we use the symbols

$$
\Delta_{\zeta} f(\zeta)=f(\zeta q)-f\left(\zeta q^{-1}\right), \quad \psi_{0}(\zeta, \alpha)=\zeta^{\alpha} \frac{1}{\zeta^{2}-1} .
$$

In the papers [25, 1] we used instead of $\psi_{0}(\zeta, \alpha)$ the function $\psi(\zeta, \alpha)=\psi_{0}(\zeta, \alpha)-\frac{1}{2} \zeta^{\alpha}$. As it has been shown in [26] on the finite lattice, either of these two functions can be used in the equations defining $\omega(\zeta, \xi)$ without changing the final result. So, we can choose the one which is more appropriate for the scaling limit.

In order to define $\omega(\zeta, \xi)$ we have to introduce the operation $\delta_{\zeta}^{-}$

$$
\delta_{\zeta}^{-} f(\zeta)=f(\zeta q)-\rho(\zeta) f(\zeta)
$$

and the convolution

$$
A \star B(\zeta, \xi)=\int_{\gamma} A(\zeta, \eta) B(\eta, \xi) d m(\eta)
$$

with measure

$$
d m(\eta)=\frac{d \eta^{2}}{\eta^{2} \rho(\eta)(1+\mathfrak{a}(\eta))} .
$$

The fundamental role is played by the dressed resolvent

$$
R_{\text {dress }}-R_{\text {dress }} \star K_{\alpha}=K_{\alpha}
$$

where $K_{\alpha}$ stands for the integral operator with the kernel $K(\zeta / \xi, \alpha)$. Introducing two more kernels

$$
f_{\text {left }}(\zeta, \xi)=\frac{1}{2 \pi i} \delta_{\zeta}^{-} \psi_{0}(\zeta / \xi, \alpha), \quad f_{\text {right }}(\zeta, \xi)=\delta_{\xi}^{-} \psi_{0}(\zeta / \xi, \alpha)
$$

we define $\omega(\zeta, \xi)$ by

$$
\frac{1}{4} \omega(\zeta, \xi)=\left(f_{\text {left }} \star f_{\text {right }}+f_{\text {left }} \star R_{\text {dress }} \star f_{\text {right }}\right)(\zeta, \xi)-\omega_{0}(\zeta, \xi)
$$

where

$$
\omega_{0}(\zeta, \xi)=-\delta_{\zeta}^{-} \delta_{\xi}^{-} \Delta_{\zeta}^{-1} \psi_{0}(\zeta / \xi, \alpha)
$$

The definition of the "primitive function" $\Delta^{-1}$ is ambiguous. Using $Z=\zeta^{1 / \nu}, H=$ $\eta^{1 / \nu}, X=\xi^{1 / \nu}$, we shall define once and forever [1]

$$
\Delta_{\zeta}^{-1} \psi_{0}(\zeta / \xi, \alpha)=\frac{1}{2 \nu} V P \int_{0}^{\infty} \psi_{0}(\zeta / \eta, \alpha) \frac{H}{H+X} \frac{d \eta^{2}}{2 \pi i \eta^{2}}
$$


where the principal value is taken with regards to the pole at $\eta^{2}=\zeta^{2}$. By this definition, we have

$$
\Delta_{\zeta}^{-1} \psi_{0}\left(q^{ \pm 1} \zeta / \xi, \alpha\right)= \pm \frac{1}{4 \nu} \frac{Z}{Z-X}+\frac{1}{2 \nu} \int_{0}^{\infty} \psi_{0}\left(\zeta e^{ \pm i 0} / \eta, \alpha\right) \frac{H}{H-X e^{ \pm i 0}} \frac{d \eta^{2}}{2 \pi i \eta^{2}}
$$

Here the left hand side is the analytic continuation of $\Delta_{\zeta}^{-1} \psi_{0}(\zeta, \alpha)$ in the variable $\frac{1}{\nu} \log \zeta$ from $\operatorname{Im} \frac{1}{\nu} \log \zeta=0$ to $\operatorname{Im} \frac{1}{\nu} \log \left(q^{ \pm 1} \zeta\right)= \pm \pi i$.

A physically interesting situation occurs when we take the scaling limit which implies in particular $\mathbf{n} \rightarrow \infty$. Here we consider two cases. We shall be very brief because detailed explanations are given in [3].

\section{Chiral CFT.}

In the homogeneous case $\zeta_{j}=1, \tau_{\mathbf{m}}=1$, the scaling limit

$$
\mathbf{n} \rightarrow \infty, \quad a \rightarrow 0, \quad \mathbf{n} a=2 \pi R, \quad \zeta=\lambda(C a)^{\nu},
$$

( $R$ and $\lambda$ are finite) describes the chiral conformal field theory [1]. The important constant $C$ is given by

$$
C=\frac{\Gamma\left(\frac{1-\nu}{2 \nu}\right)}{2 \sqrt{\pi} \Gamma\left(\frac{1}{2 \nu}\right)} \Gamma(\nu)^{\frac{1}{\nu}}
$$

2. Sine-Gordon model.

In the inhomogeneous case

$$
\zeta_{j}=\zeta_{0}^{(-1)^{j}}, \quad \tau_{\mathbf{m}}=\zeta_{0}^{(-1)^{\mathbf{m}}}
$$

the scaling limit

$$
\mathbf{n} \rightarrow \infty, \quad a \rightarrow 0, \quad \mathbf{n} a=2 \pi R, \quad \zeta_{0} \rightarrow \infty, \quad \boldsymbol{\mu}=\zeta_{0}^{-1}(C a)^{-\nu},
$$

( $R$ and $\boldsymbol{\mu}$ are finite) we describes the ${ }_{\mathrm{s}} \mathrm{G}$ model on a cylinder of radius $R$ and the coupling constant $\boldsymbol{\mu}$ (see (1.1) ).

Let us briefly recall the scaling limit of the operators $\mathbf{t}^{*}(\zeta), \mathbf{b}^{*}(\zeta)$ and $\mathbf{c}^{*}(\zeta)$ in the inhomogeneous case. The construction is not trivial, so the interested reader is referred to the papers [2, 3] for details. The local operators in the inhomogeneous case are created by coefficients of $\mathbf{b}^{*}(\zeta), \mathbf{c}^{*}(\zeta)$ developed into seria around $\zeta^{2}=$ $\zeta_{0}^{2}$ and $\zeta^{2}=\zeta_{0}^{-2}$. From these seria, after certain Bogolubov transformation, we obtain the operators $\mathbf{b}^{+*}(\zeta), \mathbf{c}^{+*}(\zeta), \mathbf{b}^{-*}(\zeta), \mathbf{c}^{-*}(\zeta)$. Their expectation values are the determinants (7.4) wherein the "two-point correlators" given by

$$
\begin{aligned}
& Z_{\mathbf{n}}\left\{\mathbf{b}^{+*}(\zeta) \mathbf{c}^{+*}(\xi)\left(q^{2 \alpha S(0)}\right)\right\}=\omega(\zeta, \xi), \\
& Z_{\mathbf{n}}\left\{\mathbf{b}^{+*}(\zeta) \mathbf{c}^{-*}(\xi)\left(q^{2 \alpha S(0)}\right)\right\}=\omega(\zeta, \xi)+\omega_{0}(\zeta, \xi), \\
& Z_{\mathbf{n}}\left\{\mathbf{b}^{-*}(\zeta) \mathbf{c}^{+*}(\xi)\left(q^{2 \alpha S(0)}\right)\right\}=\omega(\zeta, \xi)+\omega_{0}(\zeta, \xi), \\
& Z_{\mathbf{n}}\left\{\mathbf{b}^{-*}(\zeta) \mathbf{c}^{-*}(\xi)\left(q^{2 \alpha S(0)}\right)\right\}=\omega(\zeta, \xi) .
\end{aligned}
$$


The scaling limit is dictated by the behaviour of the functions $\rho, \omega$ and $\omega_{0}(\zeta, \xi)$. It can be shown (see Appendix A of [1] for very similar conclusions) that in a very general setting

$$
\begin{aligned}
& \lim _{\text {scaling }} \log \rho(\zeta) \underset{\log \zeta \rightarrow \epsilon \infty}{\simeq} \sum_{j=1}^{\infty} \rho_{\epsilon(2 j-1)} \zeta^{-\epsilon \frac{2 j-1}{\nu}}, \\
& \lim _{\text {scaling }} \frac{1}{\sqrt{\rho(\zeta)} \sqrt{\rho(\xi)}} \omega(\zeta, \xi) \underset{\substack{\log \zeta \rightarrow \epsilon \infty \\
\log \xi \rightarrow \epsilon^{\prime} \infty}}{\simeq} \sum_{j, k=1}^{\infty} \omega_{\epsilon(2 j-1), \epsilon^{\prime}(2 k-1)} \zeta^{-\epsilon \frac{2 j-1}{\nu}} \xi^{-\epsilon^{\prime 2 \frac{2 k-1}{\nu}}},
\end{aligned}
$$

where $\epsilon, \epsilon^{\prime}= \pm$. It remains to consider $\omega_{0}(\zeta, \xi)$. Recall the definition (17.10). Since we know the asymptotics of $\rho(\zeta)$ the only non-trivial thing is the asymptotics of $\Delta_{\zeta}^{-1} \psi_{0}(\zeta, \alpha)$. For studying the asymptotics it is convenient to rewrite this function in the form of a Mellin transform:

$$
\begin{aligned}
& \Delta_{\zeta}^{-1} \psi_{0}(\zeta, \alpha)=\frac{i}{4} \int_{-\infty}^{\infty} \zeta^{2 i k} \operatorname{coth} \pi\left(k+\frac{i \alpha}{2}\right) \frac{1}{\sinh 2 \pi \nu(k-i 0)} d k \\
& \underset{\zeta \rightarrow \infty}{\simeq}-\frac{i}{4 \nu} \sum_{j=0}^{\infty}(-1)^{j} \zeta^{-\frac{j}{\nu}} \cot \frac{\pi}{2 \nu}(\alpha \nu+j)-\frac{i}{2} \sum_{j=1}^{\infty} \zeta^{\alpha-2 j} \frac{1}{\sin \pi \nu(\alpha-2 j)}, \\
& \underset{\zeta \rightarrow 0}{\simeq} \frac{i}{4 \nu} \sum_{j=1}^{\infty}(-1)^{j} \zeta^{\frac{j}{\nu}} \cot \frac{\pi}{2 \nu}(\alpha \nu-j)+\frac{i}{2} \sum_{j=0}^{\infty} \zeta^{\alpha+2 j} \frac{1}{\sin \pi \nu(\alpha+2 j)} .
\end{aligned}
$$

Motivated by the above formulae we have conjectured [2, 3] that the following scaling limit exists for the operators $\mathbf{b}^{ \pm *}(\zeta), \mathbf{c}^{ \pm *}(\zeta)$ :

$$
\begin{aligned}
& \frac{1}{2} \mathbf{b}^{+*}(\zeta) \underset{\text { scaling }}{\longrightarrow} \boldsymbol{\beta}^{+*}(\zeta) \underset{\zeta \rightarrow \infty}{\simeq} \boldsymbol{\beta}^{*}(\boldsymbol{\mu} \zeta)+\left(1+O\left(\left\{\mathbf{i}_{*}\right\}\right)\right) \overline{\boldsymbol{\beta}}_{\text {screen }}^{*}(\zeta / \boldsymbol{\mu}), \\
& \frac{1}{2} \mathbf{c}^{+*}(\zeta) \underset{\text { scaling }}{\longrightarrow} \boldsymbol{\gamma}^{+*}(\zeta) \underset{\zeta \rightarrow \infty}{\simeq} \boldsymbol{\gamma}^{*}(\boldsymbol{\mu} \zeta)+\left(1+O\left(\left\{\mathbf{i}_{*}\right\}\right)\right) \overline{\boldsymbol{\gamma}}_{\text {screen }}^{*}(\zeta / \boldsymbol{\mu}), \\
& \frac{1}{2} \mathbf{b}^{-*}(\zeta) \underset{\text { scaling }}{\longrightarrow} \boldsymbol{\beta}^{-*}(\zeta) \underset{\zeta \rightarrow 0}{\simeq} \overline{\boldsymbol{\beta}}^{*}(\zeta / \boldsymbol{\mu})+\left(1+O\left(\left\{\overline{\mathbf{i}}_{*}\right\}\right)\right) \boldsymbol{\beta}_{\text {screen }}^{*}(\boldsymbol{\mu} \zeta), \\
& \frac{1}{2} \mathbf{c}^{-*}(\zeta) \underset{\text { scaling }}{\longrightarrow} \boldsymbol{\gamma}^{-*}(\zeta) \underset{\zeta \rightarrow 0}{\simeq} \overline{\boldsymbol{\gamma}}^{*}(\zeta / \boldsymbol{\mu})+\left(1+O\left(\left\{\overline{\mathbf{i}}_{*}\right\}\right)\right) \boldsymbol{\gamma}_{\text {screen }}^{*}(\boldsymbol{\mu} \zeta),
\end{aligned}
$$

where $O\left(\left\{\mathbf{i}_{*}\right\}\right), O\left(\left\{\overline{\mathbf{i}}_{*}\right\}\right)$ stand for descendants created by the local integrals of motion. We have to apologise for forgetting to write these terms in [3]. There are two explanations for that. First, in [3] we considered the one-point functions for which the descendants by the local integrals of motion are irrelevant. Second, most important, these terms are absent in the asymptotics for the chiral CFT which serves to normalise the fermionic operators. Their appearance is an artefact of mixing two chiralities, and we never use them.

The operators in the right hand side are the unique [2, 3] operators in the sG model which provide deformation of the CFT operators (6.2), 6.3). The prescription for powers of the spectral parameter are the same: they are dictated by the scaling dimensions. Looking at the asymptotics (7.17), (7.18) one concludes that the pairings of the screening operators come only from $\omega_{0}(\zeta, \xi)$. Moreover, these 
pairings are diagonal: $\boldsymbol{\beta}_{\text {screen }, j}^{*}$ couples only with $\overline{\boldsymbol{\gamma}}_{\text {screen }, j}^{*}$ and $\boldsymbol{\gamma}_{\text {screen }, j}^{*}$ couples only with $\overline{\boldsymbol{\beta}}_{\text {screen }, j}^{*}$. The corresponding pairings are easy to compute, and as a result we come to the conclusion that the screening operators can be ignored provided we postulate the formula (6.10).

An important generalisation of the above construction was proposed in [1, 3]. We have three parameters: $\alpha$, the twist $\kappa+\alpha$ for the left Matsubara transfer-matrix, and $\kappa$ for the right Matsubara transfer-matrix. By introducing the screening operators in the lattice construction it was shown that in the scaling limit we can achieve the emancipation of $\kappa+\alpha$, namely, it can be replaced by an arbitrary parameter $\kappa^{\prime}$. The basic formula (7.4) and the definitions of $\rho(\zeta)$ and $\omega(\zeta, \xi)$ remain valid in this case. A particularly nice situation occurs for $\kappa^{\prime}=\kappa$ which implies $\rho(\zeta)=1$. This case is physically relevant because it describes the one-point functions on the cylinder for the sG model [3]. But also this case is simpler from the technical point of view, and it allowed the quantitative investigation of the equation for $\omega(\zeta, \xi)$ in the CFT case [1, 28]. The price to pay for this simplification is that we can identify the action of descendants created by fermions with those created by the Virasoro generators only modulo the action of the local integrals of motion. It would be important to remove this technical obstacle. In Section 9 we shall see the first example of an exact solution for $\omega(\zeta, \xi)$ at $\rho(\zeta) \neq 1$.

\section{Infinite VOLUMe in Matsubara DiRECTION.}

Consider the limit $R \rightarrow \infty$ which corresponds to the sG model on the plane. In this case the scaling limit and the limit of the infinite volume for the lattice model commute. In other words the correct result for the functions $\rho$ and $\omega$ will be achieved if we consider the limit $\mathbf{n} \rightarrow \infty$ directly in the six-vertex case without the rescaling (7.13) or (7.15). This is so well known that we have a problem with making proper references. However, there is one less known point which we would like to underline in this section.

The results of this section is used for both left and right transfer-matrices, so, we shall denote the twist by $\theta$, assuming that it is real. In the limit $\mathbf{n} \rightarrow \infty$, the zeros of $Q(\zeta)$ densely fill $\mathbb{R}_{+}$in the plane of $\zeta^{2}$. Obviously the solutions to the equation

$$
\mathfrak{a}(\zeta)+1=0
$$

are the zeros either of $Q(\zeta)$ or of $T(\zeta)$. For the ground state all the real positive solutions to the equation (8.1) are the zeros of $Q(\zeta)$. For the excited state a finite number of real positive solutions are the zeros of $T(\zeta)$. They are conventionally called holes, and correspond to solitons in the sG language. In the sector of zero spin the number of holes is even (this is our $2 n$ ). It is necessary to introduce the same number of complex zeros of $Q(\zeta)$ in order to compensate the spin. The positions of these complex zeros are defined by the positions of the holes through the Higher Level Bethe Ansatz (HLBA) equations [31. The HLBA equations have $\left(\begin{array}{c}2 n \\ n\end{array}\right)$ solutions which count different isotopic structures for solitons-antisolitons. One more property of these complex solutions is that they do not contribute to the local integrals of motion taking care only of the isotopic structure. We shall discuss this 
in more details in Section 10. There may be an additional number of complex roots organised into strings. These strings describe breathers in the sG language, and we shall not consider them.

Changing the cycle, the DDV equation (7.5) reads as follows

$$
\begin{array}{r}
\log \mathfrak{a}(\zeta)=\log \left(\frac{d(\zeta)}{a(\zeta)}\right)-2 \pi i \nu \theta-\sum_{h} \Phi\left(\zeta / \xi_{h}\right)+\sum_{c} \Phi\left(\zeta / \xi_{c}\right) \\
-\int_{\gamma_{0}} K(\zeta / \xi) \log (1+\mathfrak{a}(\xi)) \frac{d \xi^{2}}{\xi^{2}}
\end{array}
$$

where

$$
\Phi(\zeta)=\log \left(\frac{1-q^{2} \zeta^{2}}{1-q^{-2} \zeta^{2}}\right)
$$

and $\gamma_{0}$ goes clockwise around $\mathbb{R}_{+}$. The first term in the right hand side is of order $\mathbf{n}$. This implies that $\log \mathfrak{a}(\zeta)$ is of order $\mathbf{n}$. Standard analysis shows that

$$
\begin{array}{ll}
\operatorname{Re}(\log \mathfrak{a}(\zeta))>0, & \operatorname{Im} \zeta^{2}<0, \\
\operatorname{Re}(\log \mathfrak{a}(\zeta))<0, & \operatorname{Im} \zeta^{2}>0 .
\end{array}
$$

Hence for large $\mathbf{n}$ with exponential precision in $\mathbf{n}$, only the lower part of the contour $\gamma_{0}$ contributes and we come to the linear equation:

$$
\begin{aligned}
\log \mathfrak{a}(\zeta) & -\int_{0}^{\infty} K(\zeta / \xi) \log \mathfrak{a}(\xi) \frac{d \xi^{2}}{\xi^{2}} \\
& =\log \left(\frac{d(\zeta)}{a(\zeta)}\right)-2 \pi i \nu \theta-\sum_{h} \Phi\left(\zeta / \xi_{h}\right)+\sum_{c} \Phi\left(\zeta / \xi_{c}\right)
\end{aligned}
$$

We split $\log \mathfrak{a}(\zeta)$ according to four terms in the right hand side:

$$
\log \mathfrak{a}(\zeta)=\mathbf{n} F_{\text {vac }}(\zeta)+F_{\theta}(\zeta)+F_{h}(\zeta)+F_{c}(\zeta)
$$

The first term is model dependent which means that it depends on the inhomogeneous parameters. But it does not contribute to physically relevant quantities. For example the $S$-matrices for the XXZ model and for the sG model coincide. The contributions $F_{h}(\zeta)$ and $F_{c}(\zeta)$ are well known, so we shall not write the corresponding formulae. For $F_{\theta}(\zeta)$ one finds immediately a $\zeta$-independent answer:

$$
F_{\theta}=-\pi i \theta \frac{\nu}{1-\nu}
$$


Let us consider the eigenvalue $T(\zeta)$. Due to (8.3) with exponential precision in $\mathbf{n}$, for $0<\arg \left(\zeta^{2}\right)<\pi$ we have

$$
\begin{aligned}
& \log T(\zeta)=\log (a(\zeta))+\pi i \nu \theta-\frac{1}{2 \pi i} \int_{\gamma} \Psi(\zeta / \xi) d \log (1+\mathfrak{a}(\xi)) \\
& =\log (a(\zeta))+\pi i \nu \theta-\sum_{h} \Psi\left(\zeta / \xi_{h}\right)+\sum_{c} \Psi\left(\zeta / \xi_{c}\right) \\
& -\frac{1}{2 \pi i} \int_{0}^{\infty} \frac{d}{d \xi^{2}} \Psi(\zeta / \xi) \log (\mathfrak{a}(\xi)) d \xi^{2}+O\left(e^{-c \mathbf{n}}\right),
\end{aligned}
$$

where

$$
\Psi(\zeta)=\log \left(\frac{\zeta^{2} q^{-2}-1}{\zeta^{2}-1}\right)
$$

It is easy to see that

$$
\pi i \nu \theta-F_{\theta} \cdot \frac{1}{2 \pi i} \int_{0}^{\infty} \frac{d}{d \xi^{2}} \Psi(\zeta / \xi) d \xi^{2}=0
$$

so we come to an important conclusion: in the limit $\mathbf{n} \rightarrow \infty$ with exponential precision in $\mathbf{n}, \log T(\zeta)$ is independent of the twist $\theta$. Finally a standard computation yields

$$
T(\zeta)=T_{\mathrm{vac}}(\zeta) \prod_{h} \frac{\zeta^{\frac{1}{\nu}}-\xi_{h}^{\frac{1}{\nu}}}{\zeta^{\frac{1}{\nu}}+\xi_{h}^{\frac{1}{\nu}}},
$$

where $T_{\mathrm{vac}}(\zeta)$ is independent of $\theta$. The complex zeros $\xi_{c}$ do not contribute. Returning to the picture fig. 4 we see that the dependence on $\alpha$ in the intermediate states is dropped in the limit $\mathbf{n} \rightarrow \infty$. The $\alpha$-dependence remains in the quasi-local operators $q^{-2 \alpha S(0)} \mathcal{O}_{1}$ and $q^{2 \alpha S(n)} \mathcal{O}_{2}$.

On the other hand it is clear that in this limit fig. 4 represents just the form factor decomposition on the plane. Further, in the scaling limit the rotational symmetry occurs and we obtain the usual form factor decomposition for the sG model.

\section{Evaluation of $\omega(\zeta, \xi)$ In The PRESEnCE OF SOlitons}

We want to evaluate the function $\omega(\zeta, \xi)$ which corresponds to the right part of fig.4. In that case the $T_{\mathrm{L}}(\zeta)$ is given by 8.5 and $T_{\mathrm{R}}(\zeta)=T_{\mathrm{vac}}(\zeta)$. Hence

$$
\rho(\zeta)=\prod_{j=1}^{2 n} \frac{\zeta^{\frac{1}{\nu}}-\xi_{j}^{\frac{1}{\nu}}}{\zeta^{\frac{1}{\nu}}+\xi_{j}^{\frac{1}{\nu}}},
$$

where the number of holes is $2 n$. We shall use other variables

$$
\zeta^{\frac{1}{\nu}}=Z, \quad \xi_{j}^{\frac{1}{\nu}}=B_{j}=e^{\beta_{j}} .
$$

So, in our usual notation

$$
\rho(\zeta)=\frac{P(Z)}{P(-Z)} .
$$

Later we shall use also $\eta^{\frac{1}{\nu}}=H, \xi^{\frac{1}{\nu}}=X$. 
Usually the function $\omega(\zeta, \xi)$ is found in two steps. First, we solve the equation (7.7) for $R_{\text {dress }}$ and then substitute the result into (77.9) to find $\omega(\zeta, \xi)$. However, this procedure relies heavily on the assumption of simplicity of the spectrum of $T_{\mathrm{R}}(\zeta)$. In the case $\mathbf{n} \rightarrow \infty$ this assumption is not true, the eigenvalue (8.5) corresponds to $\left(\begin{array}{c}2 n \\ n\end{array}\right)$ vectors in the sector of total spin 0 . That is why the integral operator in (7.7) is degenerate and $R_{\text {dress }}$ is not well-defined. To avoid this problem we proceed in the same way as [26] introducing the function $G(\zeta, \xi)$ which satisfies the equation

$$
G(\zeta, \xi)=\delta_{\xi}^{-} \psi_{0}(\zeta / \xi, \alpha)+\frac{1}{2 \pi i} \int_{\mathbb{R}_{+} e^{+i 0}}\left(\psi_{0}(q \zeta / \eta, \alpha)-\psi_{0}\left(q^{-1} \zeta / \eta, \alpha\right)\right) G(\eta, \xi) \frac{d \eta^{2}}{\eta^{2} \rho(\eta)}
$$

The shift $e^{+i 0}$ in (9.2) refers to the poles of $1 / \rho(\eta)$. The term $\delta_{\xi}^{-} \psi_{0}(\zeta / \xi, \alpha)$ as function of $\zeta^{2}$ has poles at $\zeta^{2}=\xi^{2}$ and $\zeta^{2}=\xi^{2} q^{2}$. Let us choose such $\xi$ that these poles do not lie on $\mathbb{R}_{+}$. Then the singular integral equation (9.2) is well-defined being supplemented with the requirement that $G(\zeta, \xi)$ is regular for $\zeta \in \mathbb{R}_{+}$.

Once $G(\zeta, \xi)$ is found the function $\omega(\zeta, \xi)$ is defined by

$$
\omega(\zeta, \xi)=\delta_{\zeta}^{-} \delta_{\xi}^{-} \Delta_{\zeta}^{-1} \psi_{0}(\zeta / \xi, \alpha)+\frac{1}{2 \pi i} \int_{\mathbb{R}_{+} e^{+i 0}} \delta_{\zeta}^{-} \psi_{0}(\zeta / \eta, \alpha) G(\eta, \xi) \frac{d \eta^{2}}{\eta^{2} \rho(\eta)} .
$$

Writing down these equations we used (8.3).

The form factors were described in terms of pairings $\left(\ell^{(n)}, L^{(n)}\right)_{\alpha}$. Now we want to solve similarly the equation (9.2). We formulate the result as Proposition, giving a necessary explanation for the precise definition of $G(\zeta, \xi)$ in the proof.

Proposition 9.1. Recall that

$$
M_{0}^{(n)}\left(S_{1}, \cdots, S_{n}\right)=\left\langle\Phi_{\alpha}\right\rangle \prod_{p=1}^{n} S_{p} \prod_{p>r}\left(S_{p}^{2}-S_{r}^{2}\right) .
$$

Consider the rational function

$$
R_{Z, X}^{(n)}\left(S_{1}, \cdots, S_{n}\right)=-\frac{1}{\nu} \frac{Z X}{Z^{2}-X^{2}} \frac{P(Z)}{P(-X)} \prod_{p=1}^{n} \frac{X^{2}-S_{p}^{2}}{Z^{2}-S_{p}^{2}} M_{0}^{(n)}\left(S_{1}, \ldots, S_{n}\right) .
$$

Then for any $\ell^{(n)}$ the function

$$
G\left(\zeta, \xi ; \ell^{(n)}\right)=\frac{\left(\ell^{(n)}, R_{Z, X}^{(n)}\right)_{\alpha}}{\left(\ell^{(n)}, M_{0}^{(n)}\right)_{\alpha}}
$$

solves the equation (9.2).

Proof. First of all we have to explain how the pairing with the rational function $R_{Z, X}(S)^{(n)}$ is understood. For polynomials $\ell(\mathfrak{s}), L(S)$ we defined the pairing as

$$
(\ell, L)_{\alpha}=\int_{\mathbb{R}-i 0} \chi(\sigma) e^{\frac{\alpha \nu}{1-\nu} \sigma} \frac{m(\mathfrak{s})}{p\left(\mathfrak{s q} \mathfrak{q}^{-2}\right)} L(S) d \sigma+\int_{\Gamma} \chi(\sigma) e^{\frac{\alpha \nu}{1-\nu} \sigma} n(\mathfrak{s}) L(S) d \sigma,
$$


where

$$
p\left(\mathfrak{s q}^{-2}\right) \ell(\mathfrak{s})=m(\mathfrak{s})+a^{-2} p(\mathfrak{s}) n\left(\mathfrak{s q}^{-4}\right)-p\left(\mathfrak{s q}^{-2}\right) n(\mathfrak{s})
$$

and $m(\mathfrak{s}), n(\mathfrak{s})$ are chosen from the requirement of convergence. Now for every $S_{p}$ the rational function $R_{Z, X}^{(n)}$ has poles at $S_{p}^{2}=Z^{2}$. Admitting the existence of these poles we define the pairing $\left(\ell^{(n)}, R_{Z, X}^{(n)}\right)_{\alpha}$ by drawing the contours of integration as follows.

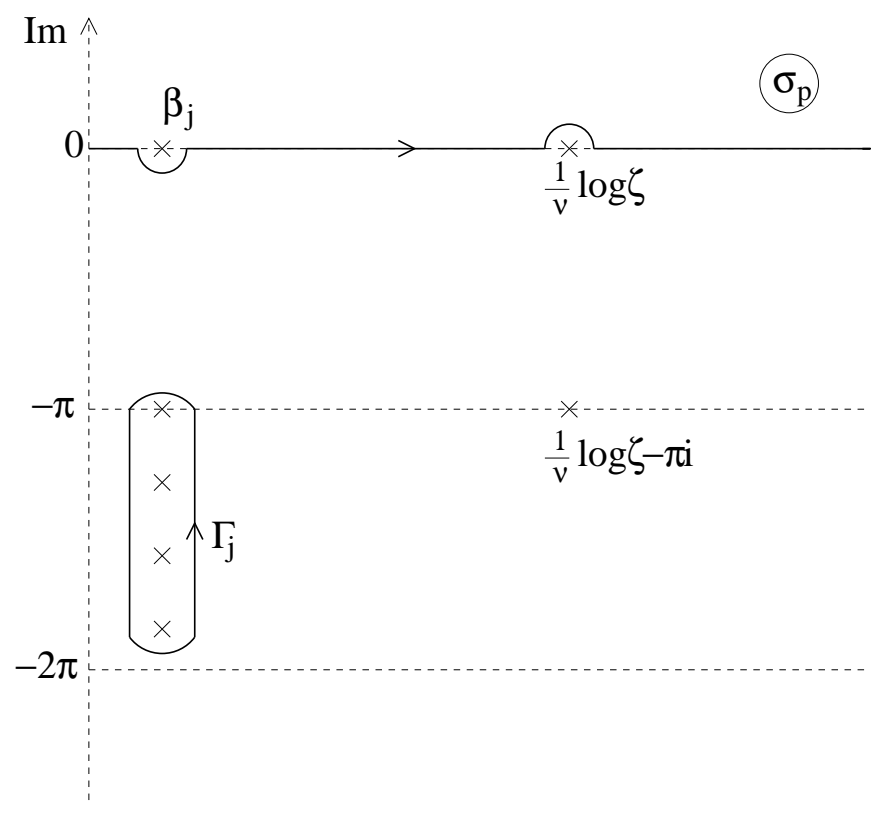

Fig.6: Contour of integration for the pairing.

The presence of the poles at $S^{2}=Z^{2}$ makes the pairing dependent on the choice of the functions $m(\mathfrak{s}), n(\mathfrak{s})$.

We immediately see that there is a trouble with this definition. The point is that the polynomials $m(\mathfrak{s})$ and $n(\mathfrak{s})$ are defined from (9.6) not uniquely. In spite of this arbitrariness the pairing was defined uniquely for polynomials $L(S)$ because we could consider the difference between two solutions to (9.6) and cancel the integrals moving the contours. For rational functions with poles at $S^{2}=Z^{2}$ this is not true because moving the contours we pick up contributions from the poles (fig. 6). Thus the pairing depends on the choice of $m(\mathfrak{s})$ and $n(\mathfrak{s})$. Still we shall show that the equation (9.2) is satisfied for any choice of $m(\mathfrak{s})$ and $n(\mathfrak{s})$ and, hence, the difference solves the homogeneous equation.

Before doing the computation of the integral in (9.2), we give some remarks. First, it is very helpful for understanding of what is going on to check the last statement in the previous paragraph directly for $n=1$ when we have only one integral over $\sigma$. Second, we shall see that the arbitrariness in question does not concern the function $\omega\left(\zeta, \xi ; \ell^{(n)}\right)$ which is the goal of our computation. The function $G\left(\zeta, \xi ; \ell^{(n)}\right)$ is an auxiliary object. It may depend on the choice of regularisation of the integrals, but the main object $\omega\left(\zeta, \xi ; \ell^{(n)}\right)$ does not. This will be proved at the end of the section. 
The last remark is regularity of $G\left(\zeta, \xi ; \ell^{(n)}\right)$ at $\zeta^{2}=\xi_{j}^{2}$. It clearly follows from fig. 6 that the integral has singularities at $\zeta=\xi_{j}$. They are simple poles, and $P(Z)$ cancels them, so, altogether $G\left(\zeta, \xi ; \ell^{(n)}\right)$ is regular at these points.

We compute

$$
\begin{aligned}
& \frac{1}{2 \pi i} \int_{\mathbb{R}_{+}}\left(\psi_{0}(q \zeta / \eta, \alpha)-\psi_{0}\left(q^{-1} \zeta / \eta, \alpha\right)\right) R_{H, X}^{(n)}\left(S_{1}, \cdots, S_{n}\right) \frac{d \eta^{2}}{\eta^{2} \rho(\eta)} \\
& =\frac{1}{2 \pi i}\left(\int_{\mathbb{R}_{+} q^{-2}}-\int_{\mathbb{R}_{+} q^{2}}\right) \psi_{0}(\zeta / \eta, \alpha) R_{-H, X}^{(n)}\left(S_{1}, \cdots, S_{n}\right) \frac{P(H)}{P(-H)} \frac{d \eta^{2}}{\eta^{2}} \\
& =-\frac{1}{2 \pi i}\left(\int_{\mathbb{R}_{+} q^{-2}}-\int_{\mathbb{R}_{+} q^{2}}\right) \psi_{0}(\zeta / \eta, \alpha) R_{H, X}^{(n)}\left(S_{1}, \cdots, S_{n}\right) \frac{d \eta^{2}}{\eta^{2}} \\
& =R_{Z, X}^{(n)}\left(S_{1}, \cdots, S_{n}\right)-\delta_{\xi}^{-} \psi_{0}(\zeta / \xi, \alpha) M_{0}^{(n)}\left(S_{1}, \cdots, S_{n}\right) \\
& -\frac{X}{P(-X)} M_{0}^{(n)}\left(S_{1}, \cdots, S_{n}\right) \sum_{p=1}^{n} S_{p}^{-1} \prod_{r \neq p} \frac{X^{2}-S_{r}^{2}}{S_{p}^{2}-S_{r}^{2}} \\
& \times\left\{\begin{array}{l}
\psi_{0}\left(\zeta S_{p}^{-\nu}, \alpha\right) P\left(S_{p}\right)-A \psi_{0}\left(\zeta\left(S_{p} Q\right)^{-\nu}, \alpha\right) P\left(-S_{p}\right), \\
\psi_{0}\left(\zeta S_{p}^{-\nu} e^{-2 \pi i \nu}, \alpha\right) P\left(S_{p}\right)-A \psi_{0}\left(\zeta\left(S_{p} Q\right)^{-\nu} e^{-2 \pi i \nu}, \alpha\right) P\left(-S_{p}\right), \quad \sigma_{p} \in \Gamma,
\end{array}\right.
\end{aligned}
$$

where we used a trivial identity:

$$
\psi_{0}\left(\zeta e^{\pi i}, \alpha\right)=A \psi_{0}(\zeta, \alpha)
$$

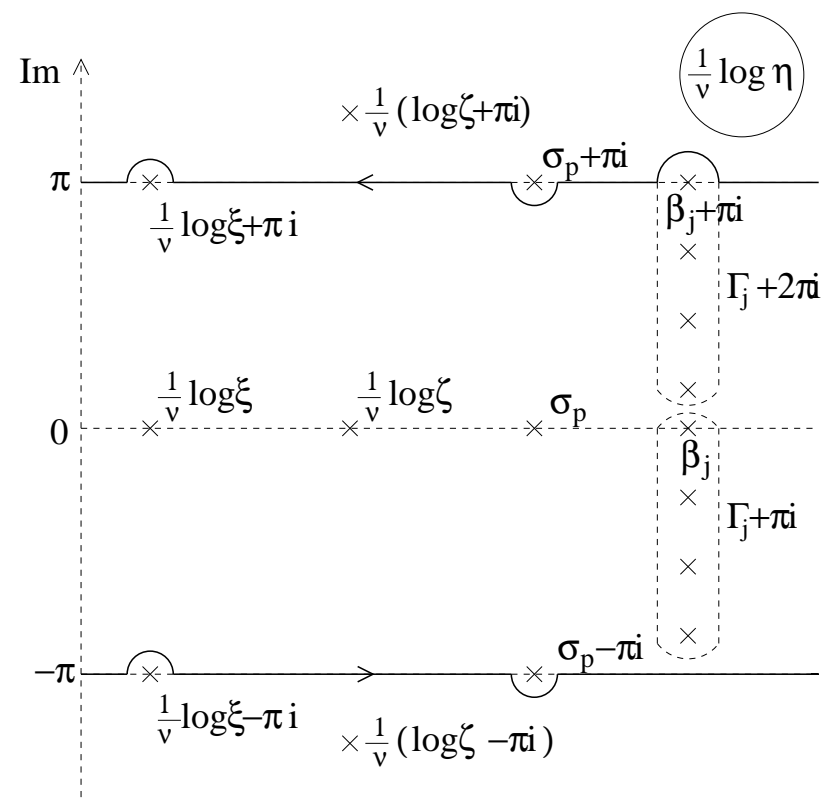


Fig.7: Poles of the integrand in (9.7).

The position of the poles of the integrand is depicted in the complex $(1 / \nu) \log \eta$ plane.

The figure fig. 7 illustrates the position of poles. The poles in sequences headed by $\beta_{j}$ and $\beta_{j}+\pi i$ effectively occur when the integration in $\sigma_{p} \in \Gamma_{j}$ is performed.

We are happy with the first two terms in the right hand side of (9.7). As for the last, containing the sum over $p$ terms, we want to show that it vanishes when the integrals over $\sigma_{p}$ are taken. Observing that in the summand for $p$ the dependence on $S_{p}$ before the curly bracket cancels out by $M_{0}^{(n)}$, one clearly sees the situation is similar to that considered in Proposition 3.4 for the $Q$-exact forms: both integrals over $\mathbb{R}$ and $\Gamma$ reduce to residue at one pole like on fig. 2. The difference with the $Q$-exact forms is that $\psi_{0}\left(\zeta S_{p}^{-\nu}, \alpha\right)$ is not a $2 \pi i$-periodic function of $\sigma_{p}$. But on the other hand there is a difference between the integrands over $\mathbb{R}$ and $\Gamma$. It is easy to see that this difference is exactly such that the residues at the fat points on fig. 2 cancel. This finishes the proof.

The difference of two solutions is proportional to

$$
\operatorname{Diff}(\zeta, \xi)=X^{-1} P(-X)\left(\left(\ell_{1}^{(n)}, R_{Z, X}^{(n)}\right)_{\alpha}\left(\ell_{2}^{(n)}, M_{0}^{(n)}\right)_{\alpha}-\left(\ell_{2}^{(n)}, R_{Z, X}^{(n)}\right)_{\alpha}\left(\ell_{1}^{(n)}, M_{0}^{(n)}\right)_{\alpha}\right) .
$$

This gives a huge but finite number of linearly independent solutions to the homogeneous equation. Indeed, each $\ell_{i}^{(n)}(i=1,2)$ is a linear combination of $\ell_{i, 1} \wedge \cdots \wedge \ell_{i, n}$. Every $\ell_{i, j}(\mathfrak{s})$ should be split into $m_{i, j}(\mathfrak{s})$ and $n_{i, j}(\mathfrak{s})$. The degree of $m_{i, j}(\mathfrak{s})$ is restricted by the requirement of convergence, and $n_{i, j}(\mathfrak{s})$ is defined modulo $p\left(\mathfrak{s q}{ }^{2}\right.$ ) (see (3.22) ). Therefore, its degree is essentially bounded by $2 n-1$. In addition $\operatorname{Diff}(\zeta, \xi)$ is a polynomial of degree $n-1$ in $X^{2}$.

Now we proceed to the computation of $\omega(\zeta, \xi)$. Using the function $G\left(\eta, \xi ; \ell^{(n)}\right)$ we compute the integral in (9.3). Since the dependence on $\eta$ and $\xi$ is solely contained in $R_{H, X}^{(n)}$, we concentrate on the computation of the integral

$$
\frac{1}{2 \pi i} \int_{\mathbb{R}_{+} e^{+i 0}} \delta_{\zeta}^{-} \psi_{0}(\zeta / \eta, \alpha) R_{H, X}^{(n)}\left(S_{1}, \ldots, S_{n}\right) \frac{d \eta^{2}}{\eta^{2} \rho(\eta)},
$$

keeping in mind that the integration with respect to the variables $S_{1}, \ldots, S_{n}$ is implied.

To this end we want first of all to transform somewhat $R_{H, X}^{(n)} / \rho(\eta)$. Do the partial fractions

$$
\begin{aligned}
& R_{H, X}^{(n)}\left(S_{1}, \cdots, S_{n}\right) / \rho(\eta) \\
& =\frac{1}{2 \nu} M_{0}^{(n)}\left(S_{1}, \ldots, S_{n}\right)\left\{-\frac{H}{H-X}+\frac{H}{H+X} \frac{P(X)}{P(-X)}\right. \\
& \left.+\frac{X}{P(-X)} \sum_{p=1}^{n} S_{p}^{-1} \frac{X^{2}-S_{r}^{2}}{S_{p}^{2}-S_{r}^{2}}\left(P\left(-S_{p}\right) \frac{H}{H-S_{p}}-P\left(S_{p}\right) \frac{H}{H+S_{p}}\right)\right\} .
\end{aligned}
$$


We call the last term in the bracket including the prefactor $\frac{1}{2 \nu} M_{0}^{(n)}\left(S_{1}, \ldots, S_{n}\right)$ the sum term. Let us transform it by using the Q-exact forms:

$$
P\left(S_{p}\right) \frac{H}{H+S_{p}} \rightarrow A P\left(-S_{p}\right) \frac{H}{H+S_{p} Q} .
$$

This is possible unless the point $\frac{1}{\nu} \log \eta-\pi i$ coincides with one of the poles inside $\Gamma$. This does not happen if $\frac{1}{\nu} \log \eta$ is slightly above all $\beta_{j}$ which can be harmlessly implied in the integral (9.3). So, our first goal is to compute the integral

$$
\begin{aligned}
& \frac{1}{2 \pi i} \int_{\mathbb{R}_{+}} \delta_{\zeta}^{-} \psi_{0}(\zeta / \eta)\left(\frac{H}{H-S_{p}}-A \frac{H}{H+S_{p} Q}\right) \frac{d \eta^{2}}{\eta^{2}} \\
& =\frac{1}{2 \pi i}\left(\int_{\mathbb{R}_{+}}-\int_{\mathbb{R}_{+} e^{-2 \pi i}}\right) \delta_{\zeta}^{-} \psi_{0}(\zeta / \eta) \frac{H}{H-S_{p}} \frac{d \eta^{2}}{\eta^{2}}=A \frac{Z}{Z-S_{p} Q}-\frac{P(Z)}{P(-Z)} \frac{Z}{Z-S_{p}} .
\end{aligned}
$$

This computation is illustrated on the figure fig. 8 where the position of the poles and the effective poles is shown. The pole in the circle does not count because of the multiplier $P\left(-S_{p}\right)$. We used $A=e^{\pi i \alpha}$ twice.

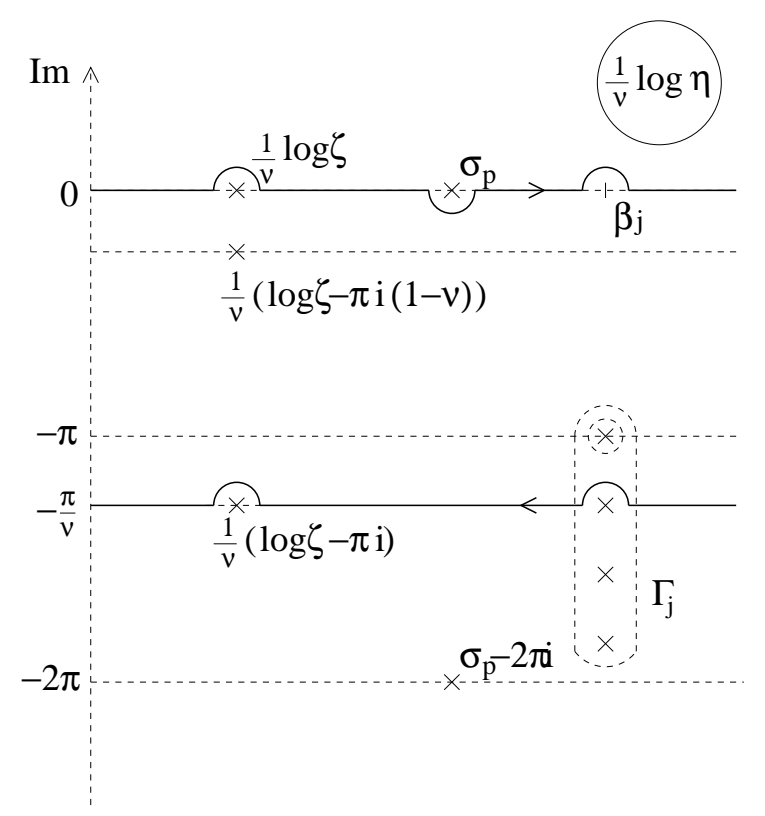

Fig.8: Contour of integration for (9.10).

We use once again the $Q$-exact forms in order to transform

$$
A \frac{Z}{Z-S_{p} Q} P\left(-S_{p}\right) \rightarrow \frac{Z}{Z-S_{p}} P\left(S_{p}\right) .
$$


Thus, the sum term without the prefactor gives rise to

$$
\frac{1}{P(-Z) P(-X)} \sum_{p=1}^{n} \frac{X}{S_{p}} \prod_{r \neq p} \frac{X^{2}-S_{r}^{2}}{S_{p}^{2}-S_{r}^{2}} \cdot \frac{Z}{Z-S_{p}}\left(P\left(S_{p}\right) P(-Z)-P\left(-S_{p}\right) P(Z)\right) .
$$

Notice that the last part is a polynomial of $Z$ and $S_{p}$. We divide it into the even and odd parts (see (5.4) for the definition of $C(Z, S)$ ):

$$
\begin{aligned}
& \frac{Z}{Z-S_{p}}\left(P\left(S_{p}\right) P(-Z)-P\left(-S_{p}\right) P(Z)\right)=-2 \nu C\left(Z, S_{p}\right) \\
& +\frac{Z}{2}\left(\frac{P\left(S_{p}\right) P(-Z)-P\left(-S_{p}\right) P(Z)}{Z-S_{p}}-\frac{P\left(-S_{p}\right) P(-Z)-P\left(S_{p}\right) P(Z)}{Z+S_{p}}\right) .
\end{aligned}
$$

For the second term the summation can be performed by the interpolation formula. We combine the result of this summation with the contribution to $\omega(\zeta, \xi)$ coming from the first term in the right hand side of (9.9):

$$
\begin{aligned}
& -\frac{1}{2 \nu} \int_{\mathbb{R}_{+} e^{+i 0}} \delta_{\zeta}^{-} \psi_{0}(\zeta / \eta)\left[\frac{H}{H-X}-\frac{H}{H+X} \frac{P(X)}{P(-X)}\right] \frac{d \eta^{2}}{2 \pi i \eta^{2}} \\
& +\frac{Z}{4 \nu P(-Z) P(-X)}\left(\frac{P(X) P(-Z)-P(-X) P(Z)}{Z-X}-\frac{P(-X) P(-Z)-P(X) P(Z)}{Z+X}\right) \\
& =-\delta_{\zeta}^{-} \delta_{\xi}^{-} \Delta_{z}^{-1} \psi_{0}(\zeta / \xi, \alpha) .
\end{aligned}
$$

We use the analytical continuation formula (7.12). Altogether we come to following nice expression

$$
\omega\left(\zeta, \xi ; \ell^{(n)}\right)=\frac{\left(\ell^{(n)}, L_{Z, X}^{(n)}\right)_{\alpha}}{\left(\ell^{(n)}, M_{0}^{(n)}\right)_{\alpha}}
$$

where the polynomial $L_{Z, X}^{(n)}$ is given by

$$
L_{Z, X}^{(n)}\left(S_{1}, \cdots, S_{n}\right)=\frac{\left\langle\Phi_{\alpha}\right\rangle}{P(-Z) P(-X)}\left|\begin{array}{cccc}
0 & C\left(Z, S_{1}\right) & \cdots & C\left(Z, S_{n}\right) \\
X & S_{1} & \cdots & S_{n} \\
X^{3} & S_{1}^{3} & \cdots & S_{n}^{3} \\
\vdots & \vdots & \vdots & \\
X^{2 n-1} & S_{1}^{2 n-1} & \cdots & S_{n}^{2 n-1}
\end{array}\right|
$$

This final result does not depend on the arbitrariness of $m(\mathfrak{s}), n(\mathfrak{s})$ because $L_{Z, X}^{(n)}\left(S_{1}, \cdots, S_{n}\right)$ is a polynomial.

\section{Equivalence of BBS and BJMS fermions.}

Let us examine the result of the previous section. For finite $\mathbf{n}$, the function $\omega(\zeta, \xi)$ is associated with each eigenstate of the left Matsubara transfer matrix. In the limit $\mathbf{n} \rightarrow \infty$, the eigenvalues are parametrized by a set of real numbers $\left\{\beta_{1}, \cdots, \beta_{2 n}\right\}$ and are $\left(\begin{array}{c}2 n \\ n\end{array}\right)$-fold degenerate. Correspondingly, for each fixed $\beta_{j}$ 's, one has to have the same number of the functions $\omega\left(\zeta, \xi ; \ell^{(n)}\right)$. 
In the process of solving the integral equation, however, we did not use any other condition on $\ell^{(n)}$ than that it is a skew-symmetric polynomial of degree at most $2 n-1$ in each variable. It enters the solution as a ratio, so the solutions of the integral equation are parametrized by points in the projective space $\mathbb{P}\left(\bigwedge^{n} V\right)$ where $V=\oplus_{j=0}^{2 n-1} \mathbb{C s}^{j}$. Let us call $\left\langle\beta_{1}, \cdots, \beta_{2 n} ; \ell^{(n)}\right|$ the actual eigenvectors of the Matsubara transfer matrix for $\mathbf{n} \rightarrow \infty$. Then the corresponding $\ell^{(n)}$ 's should be some special set of $\left(\begin{array}{c}2 n \\ n\end{array}\right)$ points of the projective space. Let us see if one can further narrow down the possibilities.

The main determinant formula (7.4) tells that

$$
\begin{aligned}
& \operatorname{det}\left(\omega\left(\zeta_{i}, \xi_{j} ; \ell^{(n)}\right)\right)_{i, j=1, \cdots k} \\
& =\frac{\left\langle\beta_{1}, \cdots, \beta_{2 n} ; \ell^{(n)}\left|\mathbf{b}^{*}\left(\zeta_{1}\right) \cdots \mathbf{b}^{*}\left(\zeta_{k}\right) \mathbf{c}^{*}\left(\xi_{k}\right) \cdots \mathbf{c}^{*}\left(\xi_{1}\right) q^{2 \alpha S(0)}\right| \mathrm{vac}\right\rangle}{\left\langle\beta_{1}, \cdots, \beta_{2 n} ; \ell^{(n)}\left|q^{2 \alpha S(0)}\right| \mathrm{vac}\right\rangle} .
\end{aligned}
$$

On the other hand, if $\ell^{(n)}$ is a pure wedge product $\ell_{0} \wedge \ell_{1} \wedge \cdots \wedge \ell_{n-1}$ of linear factors $\ell_{i}$, then from the determinant formula (9.11)-9.12) one deduces by a simple linear algebra that

$$
\operatorname{det}\left(\omega\left(\zeta_{i}, \xi_{j} ; \ell^{(n)}\right)\right)_{i, j=1, \cdots k}=\frac{\left(\ell^{(n)}, \psi_{0}^{*}\left(Z_{1}\right) \cdots \psi_{0}^{*}\left(Z_{k}\right) \chi_{0}^{*}\left(X_{k}\right) \cdots \chi_{0}^{*}\left(X_{1}\right) M_{0}^{(n)}\right)_{\alpha}}{\left(\ell^{(n)}, M_{0}^{(n)}\right)_{\alpha}}
$$

Here, as usual $Z_{j}=\zeta_{j}^{\frac{1}{\nu}}, X_{j}=\xi_{j}^{\frac{1}{\nu}}$, and we used the definitions (5.9), (5.10). This hints at the following postulate.

Postulate. The polynomials $\ell^{(n)}$ corresponding to the eigenvectors of the Matsubara transfer matrix for $\mathbf{n} \rightarrow \infty$ belong to the Grassmannian $\operatorname{Gr}(n, V) \subset \mathbb{P}\left(\bigwedge^{n} V\right)$.

We present below an argument in favour of this postulate, by invoking HLBA.

For fixed $\mathbf{n}$, the Bethe roots are either real or complex. According to [31, in the limit $\mathbf{n} \rightarrow \infty$ the real roots fill densely $\mathbb{R}_{+}$with holes which correspond to solitons with rapidities $\beta_{j}$, and the positions of the complex roots are defined by the Bethe roots of the transfer-matrix constructed from the physical S-matrix. Namely, to the vector $\left\langle\beta_{1}, \cdots, \beta_{2 n} ; \ell^{(n)}\right|$ there corresponds a Bethe vector in $\left(\mathbb{C}^{2}\right)^{\otimes 2 n}$. Let us be more precise. The two soliton S-matrix is given by

$$
\begin{gathered}
S_{i, j}\left(\beta_{i}-\beta_{j}\right)=S_{0}\left(\beta_{i}-\beta_{j}\right) \widetilde{S}_{i, j}\left(\mathfrak{b}_{i} / \mathfrak{b}_{j}\right), \\
S_{0}(\beta)=\exp \left(-i \int_{0}^{\infty} \frac{\sin (2 k \nu \beta) \sinh ((2 \nu-1) \pi k)}{k \cosh (\pi \nu k) \sinh (\pi(1-\nu) k)} d k\right),
\end{gathered}
$$

and

$$
\begin{aligned}
& \widetilde{S}_{i, j}\left(\mathfrak{b}_{i} / \mathfrak{b}_{j}\right)=\frac{1}{2}\left(I_{i} \otimes I_{j}+\sigma_{i}^{3} \otimes \sigma_{j}^{3}\right)+\frac{\mathfrak{b}_{i}-\mathfrak{b}_{j}}{\mathfrak{b}_{i} \mathfrak{q}^{-1}-\mathfrak{b}_{j} \mathfrak{q}} \cdot \frac{1}{2}\left(I_{i} \otimes I_{j}-\sigma_{i}^{3} \otimes \sigma_{j}^{3}\right) \\
& +\sqrt{\mathfrak{b}_{i} \mathfrak{b}_{j}} \frac{\mathfrak{q}^{-1}-\mathfrak{q}}{\mathfrak{b}_{i} \mathfrak{q}^{-1}-\mathfrak{b}_{j} \mathfrak{q}} \cdot\left(\sigma_{i}^{+} \otimes \sigma_{j}^{-}+\sigma_{i}^{-} \otimes \sigma_{j}^{+}\right)
\end{aligned}
$$


We define

$$
\left(\begin{array}{ll}
A(\mathfrak{t}) & B(\mathfrak{t}) \\
C(\mathfrak{t}) & D(\mathfrak{t})
\end{array}\right)_{a}=\widetilde{S}_{a, 2 n}\left(\mathfrak{t} / \mathfrak{b}_{2 n}\right) \cdots \widetilde{S}_{a, 1}\left(\mathfrak{t} / \mathfrak{b}_{1}\right)
$$

Then we are interested in constructing the eigen-covectors of the HLBA transfermatrix

$$
T^{\mathrm{HLBA}}(\mathfrak{t})=x A(\mathfrak{t})+x^{-1} D(\mathfrak{t})
$$

where $x$ is such that $|x|=1$, it is defined by $\theta$ from Section 8 . The Bethe vectors in the weight zero sector are given by the algebraic Bethe Ansatz [19]. So, the correspondence between the eigen-covector in the $\mathbf{n} \rightarrow \infty$ limit and the Bethe vector in HLBA reads as

$$
\left\langle\beta_{1}, \cdots, \beta_{2 n} ; \ell^{(n)}\right| \leftrightarrow\langle\uparrow| \prod_{j=1}^{n} C\left(\mathfrak{u}_{j}\right)
$$

where $\langle\uparrow|$ is the covector with all spins up, and $\mathfrak{u}=\left(u_{1}, \ldots,, u_{n}\right)$ satisfy the Bethe Ansatz equations. In this correspondence the eigen-covectors are parametrised by $\mathfrak{u}$. Suppose that $f_{\mathcal{O}_{\alpha}}\left(\beta_{1}, \cdots, \beta_{2 n}\right)$ is the form factor of $\mathcal{O}_{\alpha}$. Then, we have the equality

$$
\left\langle\beta_{1}, \cdots, \beta_{2 n} ; \ell^{(n)}\left|\mathcal{O}_{\alpha}\right| \mathrm{vac}\right\rangle=\langle\uparrow| \prod_{j=1}^{n} C\left(\mathfrak{u}_{j}\right) \cdot f\left(\beta_{1}, \cdots, \beta_{2 n}\right)
$$

Let us compute the right hand side by using (2.4). The definition of $w^{\epsilon_{1}, \cdots, \epsilon_{2 n}}\left(\beta_{1}, \cdots, \beta_{2 n}\right)$ is basically the same as in [7], but it has to be transposed since we consider the matrix elements between the excited state and the vacuum and not vice versa:

$$
w^{\epsilon_{1}, \cdots, \epsilon_{2 n}}\left(\beta_{1}, \cdots, \beta_{2 n}\right)=\prod_{j: \epsilon_{j}=+} C\left(\mathfrak{b}_{j}\right)|\downarrow\rangle
$$

The overall multiplier and the integral transformation involved in the formulae for the form factors are independent of the partitions. So, it is easy to see that we have to compute

$$
\begin{aligned}
& \ell_{\{u\}}^{(n)}\left(\mathfrak{s}_{1}, \cdots \mathfrak{s}_{n}\right) \\
& =\sum_{\substack{\{1, \cdots, 2 n\} \\
=I^{-} \cup I^{+}}} \ell_{I^{-} \sqcup I^{+}}^{(n)}\left(\mathfrak{s}_{1}, \cdots \mathfrak{s}_{n}\right) \frac{1}{\prod_{\substack{i \in I^{-} \\
j \in I^{+}}}\left(\mathfrak{b}_{i}-\mathfrak{b}_{j}\right)} \prod_{i \in I^{-}} \sqrt{\mathfrak{b}_{i}}\left\langle\uparrow\left|\prod_{j=1}^{n} C\left(\mathfrak{u}_{j}\right) \prod_{j: \epsilon_{j}=+} C\left(\mathfrak{b}_{j}\right)\right| \downarrow\right\rangle .
\end{aligned}
$$

The scalar product in the right hand side is the domain wall partition function given by the Izergin determinant [32], so, we face a difficult but clearly stated combinatorial problem. Surprisingly, for any set $\mathfrak{u}_{1}, \cdots, \mathfrak{u}_{n}$ (not necessarily satisfying the 
Bethe equations) we find that $\ell_{\{u\}}^{(n)}\left(\mathfrak{s}_{1}, \cdots \mathfrak{s}_{n}\right)$ belongs to the Grassmanian, namely,

$$
\begin{aligned}
& \ell_{\{u\}}^{(n)}\left(\mathfrak{s}_{1}, \cdots \mathfrak{s}_{n}\right) \\
& =c(\mathfrak{q}) \frac{\prod_{i, j=1}^{n}\left(\mathfrak{u}_{i}-\mathfrak{u}_{j} \mathfrak{q}^{2}\right)}{\prod_{i=1}^{n} \prod_{j=1}^{2 n}\left(\mathfrak{u}_{i}-\mathfrak{b}_{j} \mathfrak{q}^{2}\right)} \ell_{\{u\}, 0} \wedge \ell_{\{u\}, 1} \wedge \cdots \wedge \ell_{\{u\}, n-1}\left(\mathfrak{s}_{1}, \cdots \mathfrak{s}_{n}\right),
\end{aligned}
$$

where $c(\mathfrak{q})$ is an irrelevant constant depending only on $\mathfrak{q}$, and

$$
\begin{aligned}
& \ell_{\{u\}, j}(\mathfrak{s})=c(\mathfrak{q})^{-1} \frac{\prod_{i=1}^{n} \prod_{j=1}^{2 n}\left(\mathfrak{u}_{i}-\mathfrak{b}_{j} \mathfrak{q}^{2}\right)}{\prod_{i, j=1}^{n}\left(\mathfrak{u}_{i}-\mathfrak{u}_{j} \mathfrak{q}^{2}\right)} \\
& \times \sum_{\substack{\{1, \cdots, 2 n\} \\
=I^{-} \cup I^{+}}} \ell_{I^{-} \sqcup I^{+}, j}(\mathfrak{s}) \frac{1}{\prod_{\substack{i \in I^{-} \\
j \in I^{+}}}\left(\mathfrak{b}_{i}-\mathfrak{b}_{j}\right)} \prod_{i \in I^{-}} \sqrt{\mathfrak{b}_{i}}\left\langle\uparrow\left|\prod_{j=1}^{n} C\left(\mathfrak{u}_{j}\right) \prod_{j: \epsilon_{j}=+} C\left(\mathfrak{b}_{j}\right)\right| \downarrow\right\rangle .
\end{aligned}
$$

Notice that

$$
\ell_{I^{-} \sqcup I^{+}, j}=c_{j, 0} \mathbf{s}^{n+j}+\cdots c_{j, j} \mathbf{s}^{n}+d_{j, 1} \mathbf{s}^{n-1}+\cdots+d_{j, n},
$$

where $d_{j, k}$ depend on the partition $I^{+} \cup I^{-}$and $c_{j, k}$ do not. From this fact it is easy to see that the identity (10.7) is a necessary condition for (10.6) to hold being a part of it. The rest of equations in the identity (10.6) can be viewed as Plücker relations.

Now we see that our assumption about $\ell^{(n)}$ being in the Grassmannian fits completely with the fact that the function $\omega\left(\zeta, \xi ; \ell^{(n)}\right)$ must describe the ratio of the component of the form factor for the descendant to the one for the primary field in the basis of Bethe vectors. The formulae (10.1) and (10.2) allow us to compute the form factors of any operator for homogeneous or inhomogeneous XXZ chain. Here we shall not consider the form factors for the lattice model and proceed directly to the $\mathrm{sG}$ case.

As we have seen, for the description of the $\mathrm{sG}$ form factors, it is natural to slightly modify the operators $\psi_{0}^{*}(\zeta), \chi_{0}^{*}(\xi)$ by introducing a nontrivial block $\mathcal{A}$ in (5.21). On the other hand, when we take the scaling limit from the inhomogeneous spin chain, the lattice fermions $\mathbf{b}^{*}(\zeta), \mathbf{c}^{*}(\zeta)$ are also modified by a Bogolubov transformation involving the function

$$
\omega_{0}(\zeta, \xi)=-\delta_{\zeta}^{-} \delta_{\xi}^{-} \Delta_{\zeta}^{-1} \psi_{0}(\zeta / \xi, \alpha)
$$

If we are working with the asymptotic operators $\boldsymbol{\beta}^{*}(\zeta), \boldsymbol{\gamma}^{*}(\xi), \overline{\boldsymbol{\beta}}^{*}(\zeta), \overline{\boldsymbol{\gamma}}^{*}(\xi)$ this function contributes only to the pairings of $\boldsymbol{\beta}^{*}(\zeta)$ with $\overline{\boldsymbol{\gamma}}^{*}(\xi)$, and of $\overline{\boldsymbol{\beta}}^{*}(\zeta)$ with $\gamma^{*}(\xi)$. In these two cases we consider $\zeta \rightarrow \infty, \xi \rightarrow 0$ and $\zeta \rightarrow 0, \xi \rightarrow \infty$ respectively, and keep in the asymptotics (7.18) of $\psi_{0}(\zeta / \xi, \alpha)$ only the part going in $(\xi / \zeta)^{\frac{j}{\nu}}$ or 
$(\zeta / \xi)^{\frac{j}{\nu}}$. So, effectively $\omega_{0}(\zeta, \xi)$ is replaced either by

$$
\omega_{0,+}(\zeta, \xi)=-\delta_{\zeta}^{-} \delta_{\xi}^{-} \frac{i}{4 \nu} \sum_{j=0}^{\infty}(-1)^{j}(\xi / \zeta)^{\frac{j}{\nu}} \cot \frac{\pi}{2 \nu}(\alpha \nu+j),
$$

or by

$$
\omega_{0,-}(\zeta, \xi)=\delta_{\zeta}^{-} \delta_{\xi}^{-} \frac{i}{4 \nu} \sum_{j=1}^{\infty}(-1)^{j}(\zeta / \xi)^{\frac{j}{\nu}} \cot \frac{\pi}{2 \nu}(\alpha \nu-j) .
$$

Now it is easy to see that

$$
\omega_{0, \pm}(\zeta, \xi)=\frac{C_{ \pm}(Z, X)}{P(-Z) P(-X)}
$$

Due to this identity the effect of modifying $\mathbf{b}^{*}(\zeta)$ and $\mathbf{c}^{*}(\zeta)$ is exactly the same as the effect of modifying $\psi_{0}^{*}(\zeta), \chi_{0}^{*}(\xi)$.

Putting together all pieces of the puzzle we come to the main conclusion of this work:

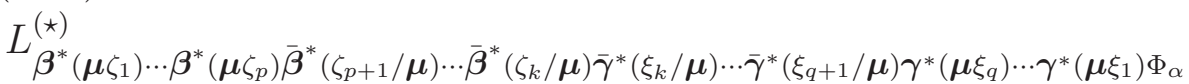

$$
\begin{aligned}
& =\psi^{*}\left(Z_{1}\right) \cdots \psi^{*}\left(Z_{p}\right) \bar{\psi}^{*}\left(Z_{p+1}\right) \cdots \bar{\psi}^{*}\left(Z_{k}\right) \bar{\chi}^{*}\left(X_{k}\right) \cdots \bar{\chi}^{*}\left(X_{q+1}\right) \chi^{*}\left(X_{q}\right) \cdots \chi^{*}\left(X_{1}\right) M_{0}^{(\star)} .
\end{aligned}
$$

So, the action of the BJMS fermions on local operators coincides with the action of the BBS fermions on towers. This exact identification of two things introduced originally for completely different reasons is one more evidence of deep self-consistency of integrable two-dimensional quantum field theory.

\section{BBS CONSTRUCTION OF NULL VECTORS}

The main achievement of the paper 17 consists in the fermionic description of null vectors. In this section we shall discuss this issue. Throughout this section we fix the number of the parameters $\beta_{j}$ to be $2 n$.

Up to now we were interested in generic $\alpha$. However, as it is clear from the discussion of the regularised integrals, something special happens at the points of resonance, i.e., at the points where the pairing (3.17) has poles. In this paper we shall consider only the resonances occurring at $\alpha=\frac{1-\nu}{\nu} m$ with $m \in \mathbb{Z}_{\geq 0}$. In the CFT language this corresponds to considering the degenerate fields which in the standard notation are

$$
\Phi_{1, m+1}=\Phi_{\frac{1-\nu}{\nu} m}
$$

We shall use both of these symbols. The polynomials $a^{n} \ell_{I^{-} \sqcup I^{+}}^{(n)}$ depend on $\alpha$ only through $a^{2}$, i.e., $\frac{1-\nu}{\nu}$-periodically. So, the form factors of the descendants $\mathcal{O}_{\alpha+m \frac{1-\nu}{\nu}}$ are expressible via the pairings $(,)_{\alpha}$ :

$$
\left(\ell_{I^{-} \sqcup I^{+}}^{(n)}(\mathfrak{s}), L^{(n)}(S)\right)_{\alpha+m \frac{1-\nu}{\nu}}=(-)^{m n}\left(\ell_{I^{-} \sqcup I^{+}}^{(n)}(\mathfrak{s}), L^{(n)}(S) \prod_{j=1}^{n} S_{j}^{m}\right)_{\alpha} .
$$

Having this in mind we shall consider all of them together. 
In the present paper we shall consider only the case of the fields $\Phi_{1,2 m}$ which we call even. According to our logic all these primary fields and their Virasoro descendants should be considered as the fermionic descendants of

$$
\Phi_{1,2}=\Phi_{\frac{1-\nu}{\nu}} .
$$

We denote this space by $\mathcal{H}_{\frac{1-\nu}{\nu}}=\oplus_{c \in \mathbb{Z}} \mathcal{H}_{\frac{1-\nu}{\nu}, c}$ where $\mathcal{H}_{\frac{1-\nu}{\nu}, c}$ denotes the sector of charge $c$.

Similarly, the case of the fields $\Phi_{1,2 m+1}$ which we call odd is reduced to the case $\alpha=0$. While the point $\alpha=\frac{1-\nu}{\nu}$ belongs to the fundamental domain (6.11), $\alpha=0$ is on its boundary, and this is a source of many complications. For that reason we decided to postpone the consideration of $\alpha=0$ till a future work. Still some technical points explained in the next subsection will be common to both even and odd cases.

11.1. Peculiar properties of form factors at $a^{2}=1$. Due to (11.1), in order to treat the case $a^{2}=1$ it is sufficient to study

$$
\left.\left(\ell_{I^{-} \sqcup I^{+}}^{(n)}, N^{(n)}(S)\right)_{\alpha}\right|_{\alpha=0}
$$

for all Laurent polynomials $N^{(n)}(S)$. Let us do that assuming for the moment that the coefficients of $N^{(n)}(S)$ are independent of $\alpha$.

Motivated by the formulae (3.15), (3.16), (3.17), let us introduce the following definition of residues for any Laurent polynomials $N(S), \ell(\mathfrak{s})$ :

$$
\begin{aligned}
& \operatorname{Res}_{+}[N]=\operatorname{res}_{S=\infty}\left(X^{+}(S) S^{-2 n} N(S) \frac{d S}{S}\right), \mathfrak{r e s}_{+}[\ell]=\operatorname{res}_{\mathfrak{s}=\infty}\left(x^{+}(\mathfrak{s}) \mathfrak{s}^{-n} \ell(\mathfrak{s}) \frac{d \mathfrak{s}}{\mathfrak{s}}\right), \\
& \operatorname{Res}_{-}[N]=\operatorname{res}_{S=0}\left(X^{-}(S) N(S) \frac{d S}{S}\right), \quad \mathfrak{r e s}_{-}[\ell]=\operatorname{res}_{\mathfrak{s}=0}\left(x^{-}(\mathfrak{s}) \ell(\mathfrak{s}) \frac{d \mathfrak{s}}{\mathfrak{s}}\right) .
\end{aligned}
$$

Extending the definition of $\operatorname{Res}_{ \pm}$as

$$
\begin{aligned}
& \operatorname{Res}_{ \pm}\left[N_{1} \wedge \cdots \wedge N_{k}\right]\left(S_{1}, \cdots, S_{k-1}\right) \\
& =\sum_{j=1}^{k}(-1)^{j-1} \operatorname{Res}_{ \pm}\left[N_{j}\right]\left(N_{1} \wedge \cdots \wedge \widehat{N}_{j} \wedge \cdots \wedge N_{k}\right)\left(S_{1}, \cdots, S_{k-1}\right),
\end{aligned}
$$

we have

$$
\begin{aligned}
& \operatorname{Res}_{ \pm}^{2}=0, \\
& \operatorname{Res}_{ \pm}\left[D_{1}[Z]\right]=0,
\end{aligned}
$$

where $D_{1}[Z]=\left.D_{A}[Z]\right|_{A=1}$. Obviously the same relations hold for $\mathfrak{r e s}_{ \pm}$defined similarly as above. Note that in the definition above, no multiple poles occur by antisymmetry.

Then using (3.15), (3.16) and (3.17), we can write the residue of the pairing as

$$
\operatorname{res}_{\alpha=0}\left(\ell^{(k)}, N^{(k)}\right)_{\alpha} d \alpha=-\frac{1-\nu}{\nu}\left(\left(\mathfrak{r e s}+\left[\ell^{(k)}\right], \operatorname{Res}_{+}\left[N^{(k)}\right]\right)_{0}-\left(\mathfrak{r e s} \mathfrak{s}_{-}\left[\ell^{(k)}\right], \operatorname{Res}_{-}\left[N^{(k)}\right]\right)_{0}\right),
$$

for any $\ell^{(k)}$ and $N^{(k)}$ whose coefficients are independent of $\alpha$. 
Consider the polynomials $\ell_{I^{-} \sqcup I^{+}, i}(i=0,1, \cdots, n-1)$. We have

$$
\ell_{I^{-} \sqcup I^{+}, 0}(\mathfrak{s})=\left(a^{-1}-a\right) \mathfrak{q}^{-2 n} p_{I^{+}}\left(\mathfrak{s q} \mathfrak{q}^{2}\right) .
$$

The rest of them have the properties $(1 \leq i \leq n-1)$

$$
\begin{aligned}
& \ell_{I^{-} \sqcup I^{+}, i}(\mathfrak{s})=a D_{a}\left[\mathfrak{s}^{i-n}\right]+O\left(\mathfrak{s}^{n-1}\right), \quad \mathfrak{s} \rightarrow \infty, \\
& \ell_{I^{-} \sqcup I^{+}, i}(\mathfrak{s})=\left(a^{-1}-a\right) C_{i}(\mathfrak{b})+O(\mathfrak{s}), \quad \mathfrak{s} \rightarrow 0,
\end{aligned}
$$

where $C_{i}(\mathfrak{b})$ are irrelevant constants depending only on $\mathfrak{b}_{j}$ 's. These follow easily from (4.3). The formulae (11.5) clearly imply that

$$
\left.\mathfrak{r e \mathfrak { S } _ { \pm }}\left[\ell_{I^{-} \sqcup I^{+}, i}(\mathfrak{s})\right]\right|_{\alpha=0}=0, \quad i=1, \cdots n-1
$$

Altogether we come to the formula

$$
\begin{aligned}
& \lim _{\alpha \rightarrow 0}\left(\ell_{I^{-} \sqcup I^{+}}^{(n)}, N^{(n)}\right)_{\alpha} \\
& =-\left.2 \pi i\left(\left(\tilde{\ell}_{I^{-} \sqcup I^{+}}^{(n-1)}, \operatorname{Res}_{+}\left[N^{(n)}\right]\right)_{\alpha}+(-\mathfrak{q})^{n} \prod_{i \in I^{-}} \mathfrak{b}_{i}^{-\frac{1}{2}} \prod_{i \in I^{+}} \mathfrak{b}_{i}^{\frac{1}{2}}\left(\tilde{\ell}_{I^{-} \sqcup I^{+}}^{(n-1)}, \operatorname{Res}_{-}\left[N^{(n)}\right]\right)_{\alpha}\right)\right|_{\alpha=0},
\end{aligned}
$$

where

$$
\tilde{\ell}_{I^{-} \sqcup I^{+}}^{(n-1)}=\ell_{I^{-} \sqcup I^{+}, 1} \wedge \cdots \wedge \ell_{I^{-} \sqcup I^{+}, n-1} .
$$

When $\alpha=0$, the monomial $S^{2 n}$ cannot be reduced to lower degree because it drops from the exact form $P(S)-A P(-S)$. So one has to relax the degree restriction (5.8) to

$$
0 \leq \operatorname{deg}_{S_{i}} N^{(n)}\left(S_{1}, \cdots, S_{n}\right) \leq 2 n
$$

An important point about $\alpha=0$ is that for certain polynomials satisfying (11.7) the pairing (11.6) vanishes. This happens if one of the following three conditions is met:

(i) Vanishing of both residues. The pairing (11.6) vanishes if

$$
\operatorname{Res}_{+}\left[N^{(n)}\right]=\operatorname{Res}_{-}\left[N^{(n)}\right]=0 .
$$

(ii) Exact form. The pairing vanishes if

$$
N^{(n)}=D[1] \wedge N^{(n-1)},
$$

where

$$
D[1]=\left.D_{A}[1]\right|_{A=1}=P(S)-P(-S) .
$$

(iii) Quantum Riemann bilinear identity. The pairing vanishes if

$$
N^{(n)}=C^{(2)} \wedge N^{(n-2)},
$$

where

$$
C^{(2)}\left(S_{1}, S_{2}\right)=C\left(S_{1}, S_{2}\right)-C\left(S_{2}, S_{1}\right)
$$


The last property (iii) needs some comments. It is a consequence of the quantum Riemann bilinear identity [16]. Consider the antisymmetric polynomial

$c^{(2)}\left(\mathfrak{s}_{1}, \mathfrak{s}_{2}\right)=p\left(\mathfrak{s}_{1}\right) \frac{\mathfrak{s}_{2} \mathfrak{q}^{2}}{\mathfrak{s}_{1}-\mathfrak{q}^{2} \mathfrak{s}_{2}}-p\left(\mathfrak{q}^{2} \mathfrak{s}_{1}\right) \frac{\mathfrak{s}_{2}}{\mathfrak{s}_{1} \mathfrak{q}^{2}-\mathfrak{s}_{2}}-p\left(\mathfrak{s}_{2}\right) \frac{\mathfrak{s}_{1} \mathfrak{q}^{2}}{\mathfrak{s}_{2}-\mathfrak{q}^{2} \mathfrak{s}_{1}}+p\left(\mathfrak{q}^{2} \mathfrak{s}_{2}\right) \frac{\mathfrak{s}_{1}}{\mathfrak{s}_{2} \mathfrak{q}^{2}-\mathfrak{s}_{1}}$

Suppose we find polynomials $r_{i}(\mathfrak{s}), s_{i}(\mathfrak{s})(i=1, \cdots, n-1)$ in the kernel of the operators

$$
\mathfrak{r e s}_{ \pm}: \mathbb{C}[\mathfrak{b}]^{2 n-1} \simeq \oplus_{j=1}^{2 n-1} \mathbb{C}[\mathfrak{b}] \mathfrak{s}^{j} \rightarrow \mathbb{C}[\mathfrak{b}]
$$

such that

$$
c^{(2)}\left(\mathfrak{s}_{1}, \mathfrak{s}_{2}\right)=\sum_{j=1}^{n-1}\left(r_{i}\left(\mathfrak{s}_{2}\right) s_{i}\left(\mathfrak{s}_{1}\right)-r_{i}\left(\mathfrak{s}_{1}\right) s_{i}\left(\mathfrak{s}_{2}\right)\right) .
$$

Then a pairing in Ker $\mathfrak{r} \mathfrak{e} \mathfrak{s}_{+} \cap \operatorname{Ker} \mathfrak{r} \mathfrak{s}_{-}$is defined by

$$
r_{i} \circ r_{j}=s_{i} \circ s_{j}=0, \quad r_{i} \circ s_{j}=\delta_{i, j} .
$$

The sets $\left\{r_{i}\right\}_{i=1}^{n-1}$ and $\left\{s_{i}\right\}_{i=1}^{n-1}$ are called half-bases. Obviously there is an action of the symplectic group $S p(2 n-2)$.

The quantum Riemann bilinear identity (see [16]) states

$$
\left(m_{1} \wedge m_{2}, C^{(2)}\right)_{0}=2 \pi i\left(m_{1} \circ m_{2}\right) .
$$

It is clear from (4.3) that for any partition $\{1, \cdots, 2 n\}=I^{-} \sqcup I^{+}$the following polynomials satisfy (11.11) for $a=1$ :

$$
r_{i}(\mathfrak{s})=\left(\mathfrak{q}^{2} \mathfrak{s}\right)^{n-i}, \quad s_{i}(\mathfrak{s})=\ell_{I^{-} \sqcup I^{+}, i}(\mathfrak{s}) .
$$

So,

$$
\ell_{I^{-} \sqcup I^{+}, i} \circ \ell_{I^{-} \sqcup I^{+}, j}=0, \quad i, j=1, \cdots, n-1,
$$

which ensures that the pairing of $\ell_{I^{-} \sqcup I^{+}, i} \wedge \ell_{I^{-} \sqcup I^{+}, j}$ and $C^{(2)}$ vanishes. The pairing of $\ell_{I^{-} \sqcup I^{+}, 0} \wedge \ell_{I^{-} \sqcup I^{+}, j}$ and $C^{(2)}$ also vanishes due to

$$
\operatorname{Res}_{+, S_{1}} C^{(2)}\left(S_{1}, S_{2}\right)=0, \quad \operatorname{Res}_{-, S_{1}} C^{(2)}\left(S_{1}, S_{2}\right)=-\frac{1}{2 \nu}\left(P\left(S_{2}\right)-P\left(-S_{2}\right)\right)
$$

and (11.9).

Before going further let us make one remark, which is supposed to be well known but probably worth being repeated. For generic $\alpha$ the polynomials $\ell_{I^{-} \sqcup I^{+}, 0} \wedge \cdots \wedge$ $\ell_{I^{-} \sqcup I^{+}, n-1}$ with different partitions of $\mathfrak{b}_{j}{ }^{\prime}$ s span the space $\bigwedge^{n} \mathbb{C}^{2 n}$ whose dimension $\left(\begin{array}{c}2 n \\ n\end{array}\right)$ coincides with that of the weight 0 subspace $\left(\mathbb{C}^{\otimes 2 n}\right)_{0}$. The latter can be interpreted in terms of the $U(1)$-symmetry of the sG model: soliton-antisoliton provide a two-dimensional representation of $U(1)$. When $\alpha=m \frac{1-\nu}{\nu}$ the polynomials $\ell_{I^{-} \sqcup I^{+}, 1}(\mathfrak{s}), \cdots, \ell_{I^{-} \sqcup I^{+}, n-1}(\mathfrak{s})$ which enter the form factor formulas are divisible by $\mathfrak{s}$, and $\mathfrak{r} \mathfrak{s}_{+}$vanishes on them. So, they are linear combinations of $2 n-2$ different monomials. Moreover, the form factors vanish if $\tilde{\ell}^{(n-1)}$ contains $c^{(2)}$ as a multiplier. Altogether, instead of $\bigwedge^{n} \mathbb{C}^{2 n}$, we have a smaller space $\bigwedge^{n-1} \mathbb{C}^{2 n-2}$ isomorphic to 
the space of the maximal irreducible representation of $S p(2 n-2)$. The dimension of this space equals

$$
\left(\begin{array}{c}
2 n-2 \\
n-1
\end{array}\right)-\left(\begin{array}{c}
2 n-2 \\
n-3
\end{array}\right)=\left(\begin{array}{c}
2 n \\
n
\end{array}\right)-\left(\begin{array}{c}
2 n \\
n-1
\end{array}\right)
$$

In the right hand side we have the Catalan number, the multiplicity of the singlet representation of the group $S L(2)$ in $\mathbb{C}^{\otimes 2 n}$. This fact agrees with the quantum group reduction [12] which states that the form factors of $\Phi_{\frac{1-\nu}{\nu} m}$ are invariant under the action of the quantum group $U_{\mathfrak{q}}\left(\mathfrak{s l}_{2}\right)$. Certainly, for generic $\mathfrak{q}$, this multiplicity is the same as in the classical case.

11.2. Null vectors for the fields $\Phi_{1,2 m}$. In the case $\alpha=\frac{1-\nu}{\nu}$ and generic $Q$ we can safely go from the odd representatives for $L^{(n)}$ to those of restricted degree and vice versa. So, following the remark at the end of Section 5 we consider the fermions defined by (5.32), which act on the space of towers with restricted degrees:

$$
0 \leq \operatorname{deg}_{S_{i}} L^{(n)}\left(S_{1}, \cdots, S_{n}\right) \leq 2 n-1 .
$$

The identification with the previous subsection goes through

$$
N^{(n)}(S)=\prod_{j=1}^{n} S_{j} \cdot L^{(n)}(S) .
$$

As already noted, we have $\left(\ell^{(k)}, L^{(n)}\right)_{\frac{1-\nu}{\nu}}=\left(\ell^{(k)}, N^{(n)}\right)_{0}$.

First, observe that

$$
C(Z, S)=\frac{1}{2 \nu} Z \cdot \frac{P(S)-P(-S)}{S}+O\left(Z^{3}\right)
$$

and

$C_{-}(Z, X)=\left.Z \cdot \frac{i}{\nu} \cot \frac{\pi}{2}\left(\alpha-\frac{1}{\nu}\right) \sigma_{2 n}(B) X^{-1}(P(X)+P(-X))\right|_{\alpha=\frac{1-\nu}{\nu}}+O\left(Z^{3}\right)=O\left(Z^{3}\right)$.

It means that, under the identification (11.14), the operator $\bar{\psi}_{1}^{*}$ acts as wedge product by an exact form. Hence by (11.9) we find a set of null vectors:

$$
\bar{\psi}_{1}^{*} \mathcal{H}_{\frac{1-\nu}{\nu},-1}=0 \text {. }
$$

We have

$$
1 \leq \operatorname{deg}_{S_{i}}\left(\prod_{j=1}^{n} S_{j} \cdot L^{(n)}\left(S_{1}, \cdots, S_{n}\right)\right) \leq 2 n,
$$

which implies that

$$
\begin{aligned}
& \operatorname{Res}_{-}\left(\prod_{j=1}^{n} S_{j} \cdot L^{(n)}\left(S_{1}, \cdots, S_{n}\right)\right)=0 \\
& \operatorname{Res}_{+}\left(\prod_{j=1}^{n} S_{j} \cdot L^{(n)}\left(S_{1}, \cdots, S_{n}\right)\right)=\prod_{j=1}^{n-1} S_{j} \cdot\left(\chi_{1}^{*} L^{(\star)}\right)^{(n-1, n)}\left(S_{1}, \cdots, S_{n-1}\right) .
\end{aligned}
$$


This gives due to $\left(\chi_{1}^{*}\right)^{2}=0$ another set of null vectors:

$$
\chi_{1}^{*} \mathcal{H}_{\frac{1-\nu}{\nu}, 1} \simeq 0 .
$$

Now we turn to the Riemann bilinear identity. Introduce the operators

$$
\mathrm{C}_{\text {even }}=\oint \psi^{*}(D) \chi(D) \frac{d D}{2 \pi i D^{3}}, \quad \bar{\complement}_{\text {even }}=\oint \bar{\psi}^{*}(D) \bar{\chi}(D) \frac{d D}{2 \pi i D^{3}} .
$$

We have for $L^{(\star)} \in \mathcal{H}_{\frac{1-\nu}{\nu},-s-2}$

$$
\left(\left(\mathrm{C}_{\text {even }}+\bar{\complement}_{\text {even }}\right) L\right)^{(n-s, n)}\left(S_{1}, \cdots, S_{n-s}\right)=-C_{\text {even }}^{(2)} \wedge L^{(n-s-2, n)}\left(S_{1}, \cdots, S_{n-s}\right),
$$

where

$$
C_{\text {even }}^{(2)}\left(S_{1}, S_{2}\right)=C\left(S_{1}, S_{2}\right) S_{1}^{-2}-C\left(S_{2}, S_{1}\right) S_{2}^{-2}
$$

The only non-trivial part of this computation is to make sure that no cross-terms occur due to the Bogolubov transform (5.19), (5.20). This follows from

$$
\left.\left(x \tau_{+}(x)-x^{-1} \tau_{-}\left(x^{-1}\right)\right)\right|_{\alpha=\frac{1-\nu}{\nu}}=0 .
$$

Since

$$
S_{1} S_{2} C_{\text {even }}^{(2)}\left(S_{1}, S_{2}\right)=-C^{(2)}\left(S_{1}, S_{2}\right),
$$

this together with (11.10) and (11.17) gives rise to new null-vectors

$$
\left(\mathcal{C}_{\text {even }}+\bar{\complement}_{\text {even }}\right) \mathcal{H}_{\frac{1-\nu}{\nu},-2} \simeq 0 \text {. }
$$

In what follows we shall be interested in right-chiral null vectors. For the operator $\Phi_{1,2}$ it is easy to identify them:

$$
\begin{array}{ll}
\chi_{1}^{*} \psi_{I^{+}}^{*} \chi_{I^{-}}^{*} M_{0}^{(\star)} \simeq 0, \quad \#\left(I^{+}\right)=\#\left(I^{-}\right)+1 \\
\mathcal{C}_{\text {even }} \psi_{I^{+}}^{*} \chi_{I^{-}}^{*} M_{0}^{(\star)} \simeq 0, \quad \#\left(I^{+}\right)=\#\left(I^{-}\right)-2, \quad 1 \notin I^{-},
\end{array}
$$

where we introduced the notation

$$
\begin{aligned}
& \psi_{I}^{*}=\psi_{a_{1}}^{*} \cdots \psi_{a_{p}}^{*}, \quad \chi_{I}^{*}=\chi_{a_{p}}^{*} \cdots \chi_{a_{1}}^{*}, \quad \bar{\psi}_{I}^{*}=\bar{\psi}_{a_{1}}^{*} \cdots \bar{\psi}_{a_{p}}^{*}, \quad \bar{\chi}_{I}^{*}=\bar{\chi}_{a_{p}}^{*} \cdots \bar{\chi}_{a_{1}}^{*}, \\
& \text { for } I=\left\{a_{1}, \cdots, a_{p}\right\}, \quad a_{1}<a_{2}<\cdots<a_{p} .
\end{aligned}
$$

We added the condition $1 \notin I^{-}$in the second formula since we do not want to count the same null-vector twice. Notice that

$$
\left[\mathcal{C}_{\text {even }}, \chi_{1}^{*}\right]=0 \text {. }
$$

Consider the right-chiral descendants of $\Phi_{1,2 m+2}$ :

$$
\psi_{I^{+}}^{*} \chi_{I^{-}}^{*} \bar{\chi}_{I_{\mathrm{odd}}(m)}^{*} M^{(\star)}, \quad \#\left(I^{+}\right)=\#\left(I^{-}\right)+m,
$$

where we recall that $I_{\text {odd }}(m)=\{1,3, \cdots, 2 m-1\}$. At first glance it is not clear how to construct the right-chiral null vectors with $\mathcal{C}_{\text {even }}+\bar{\complement}_{\text {even }}$ because $\bar{\chi}_{2 j-1}$ present in $\bar{\complement}_{\text {even }}$ might spoil the product $\bar{\chi}_{I_{\text {odd }}^{*}(m)}$. The solution was found in [17]. Consider

$$
\mathrm{C}_{\text {even }}^{m+1} \psi_{I^{+}}^{*} \chi_{I^{-}}^{*} \bar{\chi}_{I_{\text {odd }}(m)}^{*} M^{(\star)}, \quad \#\left(I^{+}\right)=\#\left(I^{-}\right)-m-2 .
$$


According to the above considerations this can be transformed

$$
\mathcal{C}_{\text {even }}^{m+1} \psi_{I^{+}}^{*} \chi_{I^{-}}^{*} \bar{\chi}_{I_{\text {odd }}(m)}^{*} M^{(\star)} \simeq\left(-\bar{\complement}_{\text {even }}\right)^{m+1} \psi_{I^{+}}^{*} \chi_{I^{-}}^{*} \bar{\chi}_{I_{\text {odd }}(m)}^{*} M^{(\star)}=0 .
$$

The latter identity is due to the fact that acting on $\bar{\chi}_{I_{\text {odd }}(m)}^{*} M^{(\star)}$ every $\bar{\complement}_{\text {even }}$ can be replaced by the finite sum $\sum_{j=1}^{m} \bar{\psi}_{2 j+1}^{*} \bar{\chi}_{2 j-1}$.

Let us summarise. The following right chiral null vectors exist for $\Phi_{(2 m+1) \frac{1-\nu}{\nu}}$ :

$$
\begin{aligned}
& \chi_{1}^{*} \psi_{I^{+}}^{*} \chi_{I^{-}}^{*} \bar{\chi}_{I_{\mathrm{odd}}(m)}^{*} M_{0}^{(\star)}, \quad \#\left(I^{+}\right)=\#\left(I^{-}\right)+m+1, \\
& \mathcal{C}_{\text {even }}^{m+1} \psi_{I^{+}}^{*} \chi_{I^{-}}^{*} \bar{\chi}_{I_{\mathrm{odd}}(m)}^{*} M_{0}^{(\star)}, \quad \#\left(I^{+}\right)=\#\left(I^{-}\right)-m-2, \quad 1 \notin I^{-} .
\end{aligned}
$$

Introduce locally the notation

$$
H_{k}=\operatorname{Span}\left\{\psi_{I^{+}}^{*} \chi_{I^{-}}^{*} M_{0}^{(\star)} \mid \#\left(I^{+}\right)=\#\left(I^{-}\right)-k, \quad 1 \notin I^{-}\right\} .
$$

It is easy to see that

$$
H_{k} \rightarrow \mathrm{e}_{\mathrm{even}}^{k} H_{-k} \quad \text { is an isomorphism . }
$$

Using (11.20) for $k=m+1$ we can combine the two kinds of the null-vectors into

$$
\bigcup_{\text {even }}^{m+1} \psi_{I^{+}}^{*} \chi_{I^{-}}^{*} \bar{\chi}_{I_{\text {odd }}(m)}^{*} M_{0}^{(\star)}, \quad \#\left(I^{+}\right)=\#\left(I^{-}\right)-m-2,
$$

if $1 \in I^{-}$we get the first kind of null-vectors, and if $1 \notin I^{-}$we get the second.

For the left descendants the situation is even simpler because $\mathcal{C}_{\text {even }}$ commutes with $\psi_{2 j-1}^{*}$ :

$$
\begin{array}{ll}
\bar{\psi}_{1}^{*} \bar{\psi}_{I^{+}}^{*} \bar{\chi}_{I^{-}}^{*} \psi_{I_{\text {odd }}(m)}^{*} M_{0}^{(\star)}, & \#\left(I^{+}\right)=\#\left(I^{-}\right)-m-1, \\
\bar{\complement}_{\text {even }} \bar{\psi}_{I^{+}}^{*} \bar{\chi}_{I^{-}}^{*} \psi_{I_{\text {odd }}(m)}^{*} M_{0}^{(\star)}, & \#\left(I^{+}\right)=\#\left(I^{-}\right)-m-2, \quad 1 \notin I^{+},
\end{array}
$$

which again can be put together as

$$
\bar{\complement}_{\text {even }} \bar{\psi}_{I^{+}}^{*} \bar{\chi}_{I^{-}}^{*} \psi_{I_{\text {odd }}(m)}^{*} M_{0}^{(\star)}, \quad \#\left(I^{+}\right)=\#\left(I^{-}\right)-m-2
$$

Actually, we have a symmetry between the left and the right chiral null vectors under the isomorphism

$$
\bar{\chi}_{2 j-1}^{*} \mapsto \psi_{2 j-1}^{*}, \quad \bar{\chi}_{2 j-1} \mapsto \psi_{2 j-1}, \quad \bar{\psi}_{2 j-1}^{*} \mapsto \chi_{2 j-1}^{*}, \quad \bar{\psi}_{2 j-1} \mapsto \chi_{2 j-1} .
$$

If $1 \in I^{-}$in (11.21) and $1 \in I^{+}$in (11.22) the identification simply follows from (11.20) with $k=m+1$. If $1 \notin I^{-}$in (11.21) and $1 \notin I^{+}$in (11.22) the proof goes as follows. Denote by $\mathcal{X}$ the image of $\overline{\mathcal{C}}_{\text {even }}$ under (11.23). The operators $\left(X, \mathcal{C}_{\text {even }},\left[X, \mathcal{C}_{\text {even }}\right]\right)$ constitute an $\mathfrak{s l}_{2}$-triple, we have the isomorphism (11.20) for $k=m+2$, and $\mathcal{C}_{\text {even }} \mathcal{X}$ is invertible on $H_{-m-2}$. So,

$$
X H_{-m-2}=X\left(\complement_{\text {even }} \mathcal{X}\right)^{-1} \complement_{\text {even }}^{m+2} H_{m+2}=\complement_{\text {even }}^{m+1} H_{m+2} .
$$




\section{Comparison of null-Vectors With CFT Results}

If we specialise to $\alpha=\alpha_{1,2 m+2}(m=0,1,2, \ldots)$ where

$$
\alpha_{1,2 m+2}=(2 m+1) \frac{1-\nu}{\nu},
$$

the Verma module $\mathcal{V}_{\alpha}$ has a singular vector at level $2 m+2$. Let $w_{2 m+2}$ be the singular vector, and $\mathcal{W}_{2 m+2}$ the submodule generated by $w_{2 m+2}$. One expects that the null vectors in the $\mathrm{sG}$ model in the previous section should give rise to a fermionic description of the singular vector $w_{2 m+2}$ and the space $\mathcal{W}_{2 m+2}$. By using the results of 11, 28, in this section we will check that it is indeed so (up to level 8 and modulo local integrals of motion). We plan to give some more details including the case of both chiralities in a separate publication.

From (6.6) one derives

$$
\boldsymbol{\beta}_{J^{+}-2 m}^{*} \boldsymbol{\gamma}_{J^{-}+2 m}^{*} \boldsymbol{\gamma}_{I_{\mathrm{odd}}(m)}^{*} \Phi_{\alpha+2 m \frac{1-\nu}{\nu}}(0) \cong C_{m}^{\prime}(\alpha) \boldsymbol{\beta}_{J^{+}}^{*} \boldsymbol{\gamma}_{J^{-}}^{*} \overline{\boldsymbol{\gamma}}_{I_{\mathrm{odd}}(m)}^{*} \Phi_{\alpha}^{(m)}(0),
$$

where $\#\left(J^{+}\right)=\#\left(J^{-}\right)+m, C_{m}^{\prime}(\alpha)=(-)^{m(m+1) / 2} \prod_{j=1}^{m} t_{2 j-1}(\alpha) C_{m}(\alpha)$, and for negative indices

$$
\boldsymbol{\beta}_{-a}^{*}=t_{a}(2-\alpha) \boldsymbol{\gamma}_{a}
$$

Using (12.1) and the results of the previous section we obtain for the space

$$
\mathcal{W}_{2 m+2}^{\text {quo }}=\mathcal{W}_{2 m+2} / \sum_{k=1}^{\infty} \mathbf{i}_{2 k-1} \mathcal{W}_{2 m+2},
$$

the following fermionic basis

$$
\left(\mathcal{C}_{m, \mathrm{even}}\right)^{m+1} \boldsymbol{\beta}_{I^{+}}^{*} \boldsymbol{\gamma}_{I^{-}}^{*} \Phi_{(2 m+1) \frac{1-\nu}{\nu}}, \quad \#\left(I^{+}\right)=\#\left(I^{-}\right)-2 m-2,
$$

where

$$
\mathcal{C}_{m, \text { even }}=\sum_{j=1}^{\infty} \boldsymbol{\beta}_{2 j+1-2 m}^{*} \boldsymbol{\gamma}_{2 j-1+2 m},
$$

with the convention (12.2).

For making comparison with CFT results, it is convenient to work with fermions $\boldsymbol{\beta}_{a}^{\mathrm{CFT} *}, \boldsymbol{\gamma}_{a}^{\mathrm{CFT} *}[1]$ normalised as

$$
\begin{aligned}
& \beta_{a}^{*}=D_{a}(\alpha) \beta_{a}^{\mathrm{CFT} *}, \gamma_{a}^{*}=D_{a}(2-\alpha) \gamma_{a}^{\mathrm{CFT} *} \\
& D_{a}(\alpha)=-\sqrt{\frac{i}{\nu}} \Gamma(\nu)^{-a / \nu}(1-\nu)^{a / 2} \frac{1}{\left(\frac{a-1}{2}\right) !} \frac{\Gamma\left(\frac{\alpha}{2}+\frac{a}{2 \nu}\right)}{\Gamma\left(\frac{\alpha}{2}+\frac{a(1-\nu)}{2 \nu}\right)} .
\end{aligned}
$$

As it has been mentioned several times, at this moment we can identify the action of $\boldsymbol{\beta}_{2 j-1}^{*}, \boldsymbol{\gamma}_{2 j-1}^{*}$ only in the quotient space

$$
\mathcal{V}_{\alpha}^{\text {quo }}=\mathcal{V}_{\alpha} / \sum_{k=1}^{\infty} \mathbf{i}_{2 k-1} \mathcal{V}_{\alpha} .
$$


As a basis in this space we shall take $P\left(\mathbf{l}_{-2}, \mathbf{l}_{-4}, \cdots\right) \Phi_{\alpha}$ where the $P$ are monomials in the even Virasoro generators ordered lexicographically. Another basis in the same space is provided by monomials in the fermions,

$$
\Psi_{a_{1}, \cdots, a_{p}, b_{1}, \cdots, b_{p}}=\boldsymbol{\beta}_{a_{1}}^{\mathrm{CFT} *} \cdots \boldsymbol{\beta}_{a_{p}}^{\mathrm{CFT} *} \boldsymbol{\gamma}_{b_{1}}^{\mathrm{CFT} *} \cdots \boldsymbol{\gamma}_{b_{p}}^{\mathrm{CFT} *} \Phi_{\alpha} .
$$

Identification of these monomials with the Virasoro basis (in $\mathcal{V}_{\alpha}^{q u o}$ ) has been given up to level 6 in [1], and at level 8 in [28]. The general structure is as follows

$$
\Psi_{\star} \equiv\left(P_{\star}^{\text {even }}\left(\mathbf{l}_{-2}, \mathbf{l}_{-4}, \cdots\right)+d_{\alpha} P_{\star}^{\text {odd }}\left(\mathbf{l}_{-2}, \mathbf{l}_{-4}, \cdots\right)\right) \Phi_{\alpha},
$$

where $d_{\alpha}=\frac{1}{6} \sqrt{(25-c)\left(24 \Delta_{\alpha}+1-c\right)}$, and the coefficients of $P_{\star}^{\text {even }}, P_{\star}^{\text {odd }}$ depend only on the central charge $c$ and on the scaling dimension $\Delta_{\alpha}$. The dependence on $c$ is polynomial while the dependence on $\Delta_{\alpha}$ is generally rational, simple poles at certain negative integers may appear.

The operator $\mathcal{C}_{m \text {,even }}$ reads now as

$$
\begin{aligned}
& \mathcal{C}_{m, \text { even }}=\sum_{j=1}^{\infty} \frac{D_{2 j-1}\left((2 m-1) \frac{1-\nu}{\nu}\right)}{D_{4 m+2 j-3}\left(2-(2 m-1) \frac{1-\nu}{\nu}\right)} \boldsymbol{\beta}_{2 j-1}^{\mathrm{CFT} *} \boldsymbol{\gamma}_{2 j+4 m-3}^{\mathrm{CFT}} \\
& -\sum_{j=1}^{m-1} \frac{t_{2 j-1}\left(2-(2 m-1) \frac{1-\nu}{\nu}\right)}{D_{2 j-1}\left(2-(2 m-1) \frac{1-\nu}{\nu}\right) D_{4 m-2 j-1}\left(2-(2 m-1) \frac{1-\nu}{\nu}\right)} \boldsymbol{\gamma}_{2 j-1}^{\mathrm{CFT}} \boldsymbol{\gamma}_{4 m-2 j-1}^{\mathrm{CFT}},
\end{aligned}
$$

Actually, after the common multiplier $\Gamma(\nu)^{\frac{4 m-2}{\nu}}$ is extracted, the remaining combinations of $\Gamma$-functions in the right hand side collapse to rational functions of $\nu$.

The following are the fermionic null vectors in $W_{2 m+2}^{\text {quo }}$ up to level 8:

$$
\begin{array}{lllll}
\mathcal{W}_{2}^{\text {quo }} & \text { level } 2 & \Psi_{1,1} & \\
& \text { level } 4 & \Psi_{3,1} & \\
& \text { level } 6 & \Psi_{5,1}, & \Psi_{1,5}-\frac{4\left(\nu^{2}-4\right)}{\nu^{2}-16} \Psi_{3,3} & \\
& \text { level } 8 & \Psi_{7,1}, & \Psi_{1,7}-\frac{9\left(\nu^{2}-4\right)}{\nu^{2}-36} \Psi_{5,3}, & \Psi_{1,3,3,1} \\
\mathcal{W}_{4}^{\text {quo }} & \text { level } 4 & \Psi_{1,3} & \\
& \text { level } 6 & \Psi_{3,3} & \\
& \text { level 8 } & \Psi_{5,3}, & \Psi_{1,3,3,1} \\
\mathcal{W}_{6}^{\text {quo }} & \text { level 6 } & \Psi_{1,5} & \\
& \text { level 8 } & \Psi_{3,5} & \\
\mathcal{W}_{8}^{\text {quo }} & \text { level 8 } & \Psi_{1,7} &
\end{array}
$$

In every module the null-vectors of lowest possible degree are singular vectors. Modulo the integrals of motion, the simplest singular vector is

$$
w_{2}=\left(\mathbf{l}_{-2}-\frac{1}{1-\nu} \mathbf{l}_{-1}^{2}\right) \Phi_{1,2} \equiv \mathbf{l}_{-2} \Phi_{1,2},
$$

which agrees with the result [1]

$$
\Psi_{1,1} \equiv \mathbf{l}_{-2} \Phi_{1,2}
$$

The next singular vector $w_{4}$ is less trivial.

$$
w_{4} \equiv\left(\frac{1}{2} \mathbf{l}_{-2}^{2}-\frac{6 \nu^{2}-16 \nu+11}{3(1-\nu)} \mathbf{l}_{-4}\right) \Phi_{1,4} .
$$


On the other hand we have for generic $\alpha$ [1]

$$
\Psi_{1,3} \equiv\left(\frac{1}{2} \mathbf{l}_{-2}^{2}+\frac{c-16-3 d_{\alpha}}{9} \mathbf{l}_{-4}\right) \Phi_{\alpha}
$$

Substituting $\alpha=\alpha_{1,4}$ we indeed find that $\Psi_{1,3} \equiv w_{4}$.

The formulae for $w_{6}$ and $w_{8}$ on one hand, and for $\Psi_{1,5}, \Psi_{1,7}$ [1, 28, on the other, are much more complicated. Still, substituting $\alpha=\alpha_{1,6}, \alpha=\alpha_{1,8}$ into the latter we find perfect agreement.

Consider the first null-vector which is not the singular vector in $\mathcal{W}_{2}$ :

$$
\mathbf{l}_{-2} w_{2} \equiv\left(\mathbf{l}_{-2}^{2}-\frac{2}{1-\nu} \mathbf{l}_{-4}\right) \Phi_{1,2}
$$

It is easy to check that $(1 / 2) \mathbf{l}_{-2} w_{2}$ coincides with the formula from [1]

$$
\Psi_{3,1} \equiv\left(\frac{1}{2} \mathbf{l}_{-2}^{2}+\frac{c-16+3 d_{\alpha}}{9} \mathbf{l}_{-4}\right) \Phi_{\alpha}
$$

specialised to $\alpha=\alpha_{1,2}$.

Proceeding in the same way we have checked that all the vectors from the above table indeed coincide with the Virasoro null-vectors. In every particular case we have to solve an overdetermined system of linear equations, and eventually find solutions. We consider this fact as a strong support to the statement that the BJMS fermions when they are identified with the BBS fermions create the null vectors in the Verma modules.

\section{Acknowledgements.}

Research of MJ is supported by the Grant-in-Aid for Scientific Research B-23340039. Research of TM is supported by the Grant-in-Aid for Scientific Research B-22340031. Research of FS is supported by SFI under Walton Professorship scheme, by RFBRCNRS grant 09-02-93106 and DIADEMS program (ANR) contract number BLAN012004. MJ and TM would like to thank for the hospitality extended by the Hamilton Mathematical Institute where a part of this work was begun.

\section{REFERENCES}

[1] H. Boos, M. Jimbo, T. Miwa, and F. Smirnov. Hidden Grassmann structure in the XXZ model IV: CFT limit. Commun. Math. Phys., 299:825-866, 2010.

[2] M. Jimbo, T. Miwa, and F. Smirnov. On one-point functions of descendants in sine-Gordon model. New Trends in Quantum Integrable Systems: Proceedings of the Infinite Analysis 09, World Scientific Publishing, Singapore, pages 117-137, 2010.

[3] M. Jimbo, T. Miwa, and F. Smirnov. Hidden Grassmann structure in the XXZ model V: sine-Gordon model. Lett. Math. Phys., 96:325-365, 2011.

[4] A. Zamolodchikov. Exact S-matrix of quantum sine-Gordon solitons. JETP Lett., 25:468-481, 1977.

[5] F. Smirnov. The general formula for solitons form factors in sine-Gordon model. J. Phys., A19:L575, 1986.

[6] A. Kirillov and F. Smirnov. A representation of the current algebra connected with the SU(2)invariant Thirring model. Phys. Lett., B 198:506-510, 1987.

[7] F. Smirnov. Form Factors in Completely Integrable Models of Quantum Field Theory. World Scientific, 1992. 
[8] Al. Zamolodchikov. Two point correlation function in scaling Lee-Yang model. Nucl. Phys., B348:619-641, 1991.

[9] S. Lukyanov. Form-factors of exponential fields in the sine-Gordon model. Mod. Phys. Lett., A12:2543-2550, 1997.

[10] A.A. Belavin, A.M. Polyakov, and A.B. Zamolodchikov. Infinite conformal symmetry in twodimensional quantum field theory. Nucl. Phys., B241:333-380, 1984.

[11] F. Smirnov. Reductions of the sine-Gordon model as a perturbation of minimal models of conformal field theory. Nucl. Phys., B 337:156-180, 1990.

[12] N. Reshetikhin and F. Smirnov. Hidden quantum group symmetry and integrable perturbations of conformal field theories. Commun. Math. Phys., 131:157-177, 1990.

[13] S. Lukyanov and A. Zamolodchikov. Exact expectation values of local fields in quantum sineGordon model. Nucl.Phys., B493:571-587, 1997.

[14] M. Jimbo and T. Miwa. Algebraic Analysis of Solvable Lattice Models, volume 85. AMS, 1995.

[15] F. Smirnov. Form-factors, deformed Knizhnik-Zamolodchikov equations and finite gap integration. Commun. Math. Phys., 155:459-487, 1993.

[16] F. Smirnov. On the deformation of Abelian integrals. Lett. Math. Phys., 36:267-275, 1996.

[17] O. Babelon, D. Bernard, and F. Smirnov. Null-vectors in integrable field theory. Commun. Math. Phys., 186:601-648, 1997.

[18] B. Feigin, M. Jimbo, M. Kashiwara, T. Miwa, and E. Mukhin and Y. Takeyama. A functional model for the tensor product of level 1 highest and level -1 lowest modules for the quantum affine algebra $U_{q}\left(\widehat{\mathfrak{s l}}_{2}\right)$. Eur. J. Combinatorics, 25:1197-1229, 2004.

[19] E. Sklyanin, L. Takhtajan, and L. Faddeev. Quantum inverse problem method. Teor. Mat. Fiz, 40:194-220, 1979.

[20] H. Boos, M. Jimbo, T. Miwa, F. Smirnov, and Y. Takeyama. Hidden Grassmann structure in the XXZ model. Commun. Math. Phys., 272:263-281, 2007.

[21] H. Boos, M. Jimbo, T. Miwa, F. Smirnov, and Y. Takeyama. Hidden Grassmann structure in the XXZ model II : Creation operators. Commun. Math. Phys., 286:875-932, 2009.

[22] V. Drinfeld. Quantum Groups. In Proceedings of the International Congress of Mathematicians, pages 798-820, Berkeley, 1990.

[23] M. Jimbo. A q-difference analogue of $U(\mathfrak{g})$ and the Yang-Baxter equation. Lett. Math. Phys., 10:63-69, 1985.

[24] V. Bazhanov, S. Lukyanov and A. Zamolodchikov. Integrable structure of conformal field theory III: the Yang-Baxter relation. Commun. Math. Phys., 200:297-324, 1999.

[25] M. Jimbo, T. Miwa, and F. Smirnov. Hidden Grassmann structure in the XXZ model III: Introducing Matsubara direction. J. Phys. A:Math.Theor., 42:304018, 2009.

[26] H. Boos and F. Göhmann. On the physical part of the factorized correlation functions of the XXZ chain. J. Phys., A42:1-27, 2009.

[27] C. Destri and H. de Vega. Unified approach to thermodynamic Bethe Ansatz and finite size corrections for lattice models and field theories. Nucl. Phys., B438:413-454, 1995.

[28] H. Boos. Fermionic basis in conformal field theory and thermodynamic Bethe Ansatz for excited states. SIGMA, 7:007, 36p, 2011.

[29] V. Fateev, D. Fradkin, S. Lukyanov, A. Zamolodchikov, and Al. Zamolodchikov. Expectation values of descendent fields in the sine-Gordon model. Nucl. Phys., B540:587-609, 1999.

[30] H. Boos, M. Jimbo, T. Miwa, and F. Smirnov. Completeness of a fermionic basis in the homogeneous $X X Z$ model. J. Math. Phys., 50:095206 (online), 2009.

[31] C. Destri and J. H. Lowenstein. Analysis of the Bethe-ansatz equations of the chiral-invariant Gross-Neveu model. Nucl. Phys., 205B:369-385, 1982.

[32] A. Izergin. Partition function of a six-vertex model in a finite volume. Sov. Phys. Dokl., 32:878-879, 1987. 
MJ: Department of Mathematics, Rikkyo University, Toshima-ku, Tokyo 171 8501, JAPAN

E-mail address: jimbomm@rikkyo.ac.jp

TM: Department of Mathematics, Graduate School of Science, Kyoto UniverSITY, KYOTO 606-8502, JAPAN

E-mail address: tmiwa@math.kyoto-u.ac.jp

FS2: Hamilton Mathematical Institute and School of Mathematics, Trinity ColLege, Dublin 2, Ireland

Laboratoire de Physique Théorique et Hautes Energies, Université Pierre et Marie Curie, Tour 13, $4^{\text {er }}$ Étage, 4 Place Jussieu 75252 Paris Cedex 05, France

E-mail address: smirnov@lpthe.jussieu.fr

\footnotetext{
${ }^{2}$ Membre du CNRS
} 